\title{
A COMPACTIFICATION OF THE UNIVERSAL PICARD VARIETY OVER THE MODULI SPACE OF STABLE CURVES
}

\author{
LUCIA CAPORASO
}

\section{CONTENTS}

0. Introduction and summary

1. Preliminaries

2. Construction of $\overline{P_{d, g}}$

3. What is the multidegree of a GIT-semistable curve?

4. Combinatorial considerations

5. The fibers of $\psi_{d}$

6. Conclusions

7. Examples

8. The moduli problem

References

\section{INTRODUCTION AND SUMMARY}

0.1. Statement of the problem. In this paper we construct a geometrically meaningful compactification for the relative degree- $d$ Picard variety associated to a family of stable curves.

More precisely, let $\mathscr{Y} \rightarrow B$ be a (proper and flat) family of stable curves of genus $g$ and let $\mathscr{J}_{\mathscr{Y} / B} \rightarrow B$ be the corresponding family of Jacobians; we want to answer the following question: does there exist a compactification of $\mathscr{J}_{\mathscr{g} / B}$ over $B$, which is natural (in a sense that we make precise below), and what does it look like?

We will now formulate the problem which we will study. We will work over an algebraically closed field $k$. Let $M_{g}$ be the moduli space of smooth curves of genus $g, g>2$, let $\bar{M}_{g}$ be its Deligne-Mumford compactification via stable curves, and let $M_{g}^{0}$ be the locus of automorphism-free nonsingular curves. If $X$ is a stable curve of genus $g$, we will denote by $[X]$ the point of $\bar{M}_{g}$ corresponding to its isomorphism class.

Received by the editors March 13, 1993.

1991 Mathematics Subject Classification. Primary 14D20, 14H10, 14H40, 14L30; Secondary $32 \mathrm{G} 20$.

The author was partially supported by a Sloan Dissertation Fellowship. 
For every integer $d$, there exists a so-called "Universal Picard variety of degree $d^{\prime \prime}, P_{d, g}$, over $M_{g}^{0}$ (sometimes denoted in the literature by $J_{g}^{d}$ or by $\mathrm{Pic}^{d, g}$ ), whose fiber over the point $[X]$ is identified with the variety of isomorphism classes of line bundles of degree $d$ on the curve $X . P_{d, g}$ coarsely represents the relative Picard functor of degree $d$ with respect to the universal family $\mathscr{C}_{g}$ over $M_{g}^{0}$; it is also known that $P_{d, g}$ is a smooth and irreducible quasi-projective variety and that $P_{d, g} \cong P_{d^{\prime}, g}$ if and only if there exists an integer $n$ such that $d \pm d^{\prime}=n(2 g-2)$. Finally, Mestrano and Ramanan [MR] proved that $P_{d, g}$ is a fine moduli space (i.e., there exists a Poincare line bundle over $\left.P_{d, g} \times{ }_{M_{g}^{0}} \mathscr{C}_{g}^{0}\right)$ if and only if $(d-g+1,2 g-2)=1$.

Our goal is to construct a projective scheme $\overline{P_{d, g}}$ with a surjective (and proper) morphism to $\bar{M}_{g}$

$$
\phi_{d}: \overline{P_{d, g}} \longrightarrow \bar{M}_{g}
$$

such that the preimage of $M_{g}^{0}$ is isomorphic to $P_{d, g}$.

Then we will describe the fiber of $\phi_{d}$ over any curve $[X] \in \bar{M}_{g}$ (denoted by $\overline{P_{d, X}}$ ) as a good compactification of the generalized Jacobian of $X$ (when $X$ has trivial automorphism group).

0.2. Compactifying the generalized Jacobian. Recall that if $X$ is a reduced, nodal, connected curve, having $\delta$ nodes and $\gamma$ irreducible components, the generalized Jacobian of $X, J_{X}$, is a smooth, commutative, algebraic group, whose points are identified with isomorphism classes of line bundles having degree 0 on each component.

Let $\nu: X^{\nu} \rightarrow X$ be the normalization of $X$. Then we have the exact sequence

$$
0 \longrightarrow\left(k^{*}\right)^{m} \longrightarrow J_{X} \stackrel{\nu^{*}}{\longrightarrow} J_{X^{\nu}} \longrightarrow 0
$$

where the integer $m$ is a combinatorial invariant of the curve: $m=\delta-\gamma+1$.

The problem of compactifying the generalized Jacobian of a fixed singular curve has been explicitly studied for the first time by Igusa, around 1950 . Since then, there has been much progress and, at the moment, good models for the compactified Jacobian are available in a large number of cases. There is a fairly rich literature on the subject, and our list of references is by no means complete.

A "good" compactification of the generalized Jacobian $J_{X}$ should be a projective $g$-dimensional scheme, containing $J_{X}$. Its points should correspond to geometric objects and there should be a natural action of $J_{X}$ on it. Finally, this compactification should be, in some sense, intrinsic, that is, invariant under suitable specialization.

When it comes to compactifying families of Jacobians for nodal curves, very little is known. The situation can be briefly summarized as follows: there is a solution for some particular families of irreducible curves, which is given by compactifying the Picard functor via the functor of torsion-free, coherent sheaves of rank 1. More precisely, for an integral curve, the moduli space of 
torsion-free, coherent sheaves having rank 1 and fixed Euler characteristic turns out to be a projective scheme, and so does its relative analogue over certain families. Such an approach was first suggested by Mayer and Mumford, and the complete construction was carried out by D'Souza (cf. [Ds]).

This method does not give a good solution for reducible curves, because in this case such a functor is no longer separated. In [OS], Oda and Seshadri considered the case of a fixed nodal (possibly reducible) curve, and constructed a number of compactifications for its generalized Jacobian by considering torsionfree sheaves of rank 1 together with some extra structure, containing the combinatorial data of the curve. Their techniques have been generalized by Ishida (cf. [Is]) to some special families of nodal curves. Their construction, as well as D'Souza's, applies Gcometric Invariant Theory on a set-up which uses the Grothendieck Quot schemes. We also use Geometric Invariant Theory in our construction, but the set-up from which we start is different, and the points of our compactification are described in a new way.

What we will do is to compactify the generalized Jacobian in such a way that its boundary points are described in terms of line bundles of degree $d$ on certain curves; these curves will be in a larger class than stable curves, but they will still be semistable (in the sense of Deligne and Mumford).

This approach will yield a natural compactification for the case of a reducible curve $X$, which will "periodically" depend on the degree and which will be denoted by $\overline{P_{d, X}}$. In such a case, we certainly do not expect the compactified Jacobian to contain an open dense subset isomorphic to $J_{X}$. This will in general not be true, unless $X$ is irreducible. What we do expect is that $\overline{P_{d, X}}$ have finitely many irreducible components, each one containing a dense subset isomorphic to $J_{X}$.

The reason is that, although the Picard functor of degree $d$ is not separated (in fact, it is easy, for example, to construct a family of line bundles over the punctured disc which has infinitely many nonisomorphic limits), we can construct a new separated functor, by means of a stronger equivalence relation on line bundles of fixed degree on a fixed curve $X$ (cf. Section 8). As we shall see, this will provide an upper bound on the number of irreducible components of our compactified Jacobian. Such an upper bound will not depend on the degree, being only a function of the dual graph of the curve, and will be denoted by $\mu(X)$.

0.3. Construction of $\overline{P_{d, g}}$. As we said, we will describe $\overline{P_{d, g}}$ in terms of line bundles on some DM-semistable curves; a natural place to look is the Hilbert scheme Hilb $_{r}^{p(x)}$ parametrizing closed subschemes of a fixed projective space $\mathbb{P}^{r}$, having fixed Hilbert polynomial $p(x)=d x-g+1$, with $r=d-g$ and $d$ sufficiently large.

There is a natural action of $P G L(r+1)$ on such a Hilbert scheme, and we will have to divide by that action. We are faced here with a geometric invariant theory situation, in fact we will construct $\overline{P_{d, g}}$ as the geometric invariant theory quotient of the action of a group over a suitable scheme. 
The first problem one has to solve, when dealing with an algebraic group $G$ acting on a projective scheme $\operatorname{Proj}(S)$, is to linearize the group action; this means, to have the group consistently act on the coordinate ring of the scheme. In fact, once one has a (compatible) action of $G$ on $S$, the natural candidate for the quotient is the projective scheme $\operatorname{Proj}\left(S^{G}\right)$, where $S^{G}$ is the (graded) ring of elements of $S$ which are invariant under the action of $G$. It is exactly to be able to find a linearization, that we will consider the action of the group $S L(r+1)$ instead of $P G L(r+1)$. From now on, we will denote $S L(r+1)$ simply by $G$.

For any $d \geq 20(g-1)$, it is possible to choose an embedding of $\mathrm{Hilb}_{r}^{p(x)}$ in a Grassmannian in such a way that the semistable points for the correspondingly linearized action of $G$ can be geometrically described: a theorem of Gieseker (cf. [G] or 1.4) shows that they correspond to Deligne-Mumford semistable curves. Let us call $H_{d}$ this set,

$$
\begin{aligned}
H_{d}=\left\{h \in \mathrm{Hilb}_{r}^{p(x)}:\right. & h \text { is } G \text {-semistable } \\
& \text { and the corresponding curve is connected }\} .
\end{aligned}
$$

The fundamental theorems of geometric invariant theory allow us to conclude that the GIT-quotient $H_{d} / G$ exists as a projective scheme.

Now, combining some fundamental properties of Deligne-Mumford stable curves with standard geometric invariant theory arguments, we can prove the following.

Theorem. Let $d \geq 20(g-1), g \geq 3$. The projective scheme $H_{d} / G$ is endowed with a surjective morphism

$$
\varphi_{d}: H_{d} / G \rightarrow \bar{M}_{g}
$$

such that $\varphi_{d}^{-1}\left(M_{g}^{0}\right)=P_{d, g}$.

We will naturally set

$$
\overline{P_{d, g}}:=H_{d} / G \text { and } \overline{P_{d, X}}:=\varphi_{d}^{-1}([X]) .
$$

The above is Theorem 2.1 .

Although the structure of $\overline{P_{d, g}}$ and $\overline{P_{d, X}}$ will depend on $d$, the properties stated in the next theorem (proved in Section 3) hold for all $d \geq 20(g-1)$.

Theorem. Let $d \geq 20(g-1)$ and $g \geq 3$. Then

(1) The projective scheme $\overline{P_{d, g}}$ is reduced, irreducible, and Cohen-Macaulay.

(2) The proper, surjective morphism

$$
\phi_{d}:{\overline{P_{d, g}}}^{\longrightarrow} \bar{M}_{g}
$$

is flat over the locus of stable curves with trivial automorphism group. The preimage of $M_{g}^{0}$ under $\phi_{d}$ is isomorphic to $P_{d, g}$.

(3) $\forall[X] \in \bar{M}_{g}, \bar{P}_{d, X}$ is a projective, connected scheme having at most $\mu(X)$ irreducible components. Moreover, if $X$ has trivial automorphism group, $\overline{P_{d, X}}$ 
is reduced and its smooth locus is isomorphic to the disjoint union of a finite number of copies of $J_{X}$.

The number $\mu(X)$ is the upper bound we talked about in 0.2. To prove this result, we have to overcome the difficulties that arise when dealing with reducible curves. The key observation is that a (reducible) curve in $\mathbb{P}^{r}$ should have semistable Hilbert point when its multidegree is "balanced" or "proportional" to its multigenus. ${ }^{1}$ More precisely, let us denote by $\operatorname{hilb}(X) \in$ Hilb $_{r}^{p(x)}$ the Hilbert point of a curve $X$ in projective space. Then we can prove the following

Theorem (Basic Inequality). Assume $d \geq 20(g-1)$ and $g \geq 3$. Let $X \subset \mathbb{P}^{r}$ be a projective curve such that $\operatorname{hilb}(X) \in H_{d}$, and let $Y \subset \mathbb{P}^{r}$ be any complete subcurve of $X$ having arithmetic genus $g_{Y}$ and intersecting the remaining components of $X$ in $k$ points. Then the degree of $Y, d_{Y}$, satisfies the following inequality:

$$
\left|d_{Y}-\frac{d}{(g-1)}\left(g_{Y}-1+k / 2\right)\right| \leq k / 2 \text {. }
$$

Conversely, if $X \subset \mathbb{P}^{r}$ is a nondegenerate, nodal, connected curve of genus $g$ and degree $d$ such that all of its subcurves satisfy the above inequality, then the Hilbert point of $X$ is semistable.

The proof is in Sections 3.1 and 6.1. The hard part is to show that if the basic inequality is satisfied for all subcurves of $X$, then the Hilbert point of $X$ is $G$-semistable. This is done indirectly, that is, without actually applying any stability criterion; rather the proof uses the semistable replacement property (or, the valuative criterion for properness) together with arguments of combinatorial flavor. This can be done thanks to a theorem of Mumford and Gieseker (whose proof is, in turn, direct) to the effect that the Hilbert point of a smooth, nondegenerate, nonspecial curve in $\mathbb{P}^{r}$ is stable, provided that the degree of the curve is higher than twice its genus (cf. Section 1.4).

0.4 . The "best" compactification. Now let $X$ be a nodal curve, having $\gamma$ irreducible components $C_{i}, i=1, \ldots, \gamma$, and let us also assume that $X$ has no nontrivial automorphisms. There is a natural equivalence relation among all the multidegrees summing to a fixed $d$, which measures the nonseparatedness of the relative Picard functor. More precisely, if $\mathscr{Z} \rightarrow \Delta_{t}$ is any smooth deformation of $X$ over the disc $\Delta_{t}$, one can twist a line bundle $\mathscr{L}$ on $\mathscr{Z}$ by any line bundle of type $\mathscr{O}_{\mathscr{Q}}\left(\sum_{n=1}^{\gamma} r_{n} C_{n}\right), r_{i} \in \mathbb{Z}$, having the only effect of changing the restriction of $\mathscr{L}$ to $X$. This gives us an example of the phenomenon we already mentioned: the family $\mathscr{Z}$, with the polarization given by $\mathscr{L}$ over the punctured disc, has infinitely many nonisomorphic limits.

Notice also that, for any fixed linear combination $\sum_{n=1}^{\gamma} r_{n} C_{n}$, the isomorphism class of the line bundle $\mathscr{O}_{\mathscr{Z}}\left(\sum_{n=1}^{\gamma} r_{n} C_{n}\right) \otimes \mathscr{O}_{X}$ depends on $\mathscr{X}$, while its

\footnotetext{
${ }^{1}$ If $C_{1}, \ldots, C_{y}$ are the irreducible components of $X \subset \mathbb{P}^{\prime \prime}$, we will define its multidegree to be $\underline{d}=\left(d_{1}, \ldots, d_{\gamma}\right)$ with $d_{i}=\operatorname{deg} C_{i}$ and its multigenus $\left(g_{1}, \ldots, g_{\gamma}\right)$ with $g_{i}$ equal to the arithmetic genus of $C_{i}$.
} 
multidegree does not. This way we are led to consider multidegree classes; the total number of them will only depend on the combinatorial data of $X$, and it will be denoted by $\mu(X)$. Under this perspective, Theorem indicates how to choose representatives for the multidegree classes.

There is an injective map from the set of irreducible components of $\overline{P_{d, X}}$ to the set of such multidegree classes; therefore $\mu(X)$ is actually an upper bound on the number of components of $\overline{P_{d, X}}$. Specifically, it turns out that, if $X$ is irreducible, $\overline{P_{d, X}}$ will also be irreducible and $\overline{P_{d, X}} \cong \overline{P_{d^{\prime}, X}}, \forall d, d^{\prime}$. If $X$ is reducible and does not have a separating node ${ }^{2} \frac{P_{d, X}}{P^{2}}$ might be reducible with each component corresponding in a natural way to a fixed multidegree $\underline{d}$, determined by the Basic Inequality. In other words, for every irreducible component $Z$ of $\overline{P_{d, X}}$, there exists a unique multidegree $\underline{d}$ such that $Z$ contains an open subset which is identified with $\mathrm{Pic}_{X}^{\frac{d}{X}}$, the variety of isomorphism classes of line bundles of multidegree $\underline{d}$ on $X$.

The irreducible components of the compactified Jacobian $\overline{P_{d, X}}$ are therefore indexed by a subset of multidegree classes; moreover, we will find models of compactified Jacobian having exactly $\mu(X)$ components. It is precisely in this case, occurring for the "general" choice of $d$, that such compactification of $J_{X}$ can be described as the scheme representing a geometrically described functor, and we will therefore say that such $\overline{P_{d, X}}$ are of geometric type. We refer to Section 8 for all the details.

A natural question is whether there are values of $d$ for which the fiber of $\overline{P_{d, g}}$ over every $[X]$ in $\bar{M}_{g}$ has $\mu(X)$ components. This is equivalent to asking whether there are values of $d$ such that $\overline{P_{d, g}}$ is a geometric quotient for the action of $G$ on $H_{d}$ (i.e., $H_{d}$ only contains stable points).

The next result is an answer to this question.

Proposition. The points in $H_{d}$ are all stable for the action of $G$ if and only if $(d-g+1,2 g-2)=1$. In this case the singularities of $\overline{P_{d, g}}$ are all of finite quotient type and for every $[X]$ in $\bar{M}_{g}^{0}$ the compactified Jacobian $\overline{P_{d, X}}$ is of geometric type; moreover $\overline{P_{d, g}}$ coarsely represents the functor $\overline{\mathscr{P}_{d, g}}$.

If $\overline{P_{d, g}}$ satisfies the condition of this theorem, we will say that $\overline{P_{d, g}}$ is a geometric or nondegenerate compactification of the universal Picard variety $P_{d, g}$.

If $X$ has trivial automorphism group and it is not of compact type, $\overline{P_{d, X}}$ has less than $\mu(X)$ components exactly when there are properly semistable points in the preimage of $[X]$ in $H_{d}$. If that happens, some degree classes will not be represented by any irreducible component of $\overline{P_{d, X}}$; instead, they will correspond to some positive codimension loci.

\footnotetext{
${ }^{2}$ For example, for curves of compact type $\overline{P_{d, X}}$ will always be irreducible and isomorphic to $J_{X}$, cf. 7.2 .
} 
0.5. Examples. Let us consider, as an example, the case of an automorphismfree curve $X$ having two smooth irreducible components meeting at $k \geq 2$ points. For the general choice of $d, \overline{P_{d, X}}$ will be of geometric type and it will have $k$ irreducible components, all of dimension $g$. For some special values of $d$, for example, $d=(2 n+1)(g-1), \overline{P_{d, X}}$ will have $k-1 g$-dimensional components.

A detailed description of this example is in 7.3. Here we want to say a few more words for the case $k=3$, with the purpose of giving an idea of how the boundary of $\overline{P_{d, X}}$ will be described.

As we said, there are, up to isomorphism, exactly two models for $\overline{P_{d, X}}$ : the "general" or "canonical" model, having three components, and the "special" or "degenerate" model, having two components. The generalized Jacobian of such a curve is a $\left(k^{*}\right)^{2}$-bundle over the Jacobian of its normalization, and we will find boundary loci in codimensions 1 and 2. In fact, an open subset of any irreducible component of the boundary (having codimension 1 in $\overline{P_{d, X}}$ ) can be identified with the generalized Jacobian of the curve obtained by desingularizing $X$ at one particular node $N$. Each (codimension 2 in $\overline{P_{d, X}}$ ) irreducible component of the boundary of such locus will be identified with the Jacobian of the curve of compact type obtained by desingularizing $X$ at two given nodes, one of which is of course $N$.

In the canonical model, there will be three such codimension 2 loci, corresponding to the three nodes of $X$. In the degenerate model, all such loci coincide, and the (irreducible) codimension 2 corresponding locus can be better described as the Jacobian of the normalization of $X$.

This provides a stratum by stratum description of $\overline{P_{d, X}}$. A global way of describing $\overline{P_{d, X}}$ is to consider the DM-semistable curve $X^{\ell}$ (which will be called the "ladder of $X$ "), which is obtained from $X$ by replacing each node by a $\mathbb{P}^{1}$ so that $X^{\ell}$ has five irreducible components, three of which are smooth rational curves. Then, the geometric $\overline{P_{d, X}}$ is a parameter space for "equivalence" classes of line bundles on $X^{\ell}$, where such an equivalence relation amounts roughly to moding out by twisting. The details of this example can be found in 7.3; here we want to conclude by mentioning that, although for every DM-stable curve $X$, and for all $d$, the singular locus of $\overline{P_{d, X}}$ is generically normal crossing, already in this particular example $\overline{P_{d, X}}$ will have nonnormal crossing singularities in the codimension 2 loci.

\section{Preliminaries}

We will compactify the universal Picard variety using Geometric Invariant Theory (GIT) in a way that has nowadays become standard for this type of problem. We will start with a brief summary of the main results of Geometric Invariant Theory and then we will illustrate one of their most important applications: the construction of a compactification of the moduli space of smooth curves of fixed genus, as carried out by Mumford and Gieseker. In doing this, 
we will also have the opportunity to recall a number of facts that will be needed for our construction of $\overline{P_{d, g}}$.

1.1. Terminology. As we said, we will work over an algebraically closed field $k$. Curves will always be assumed to be projective over $k$. By the genus of a curve $Y$ we will mean the arithmetic genus, and we will usually denote it by $g_{Y}$. If $Y \subset \mathbb{P}^{r}$ we will denote by $d_{Y}$ its degree.

"DM-stable [semistable] curve" will stand for a stable [semistable] curve in the sense of Deligne and Mumford, that is, a reduced, connected, curve having only ordinary double points as singularities and such that every smooth rational component meets the remaining components in at least three [two] points.

Let $X$ be a DM-semistable curve and let $E$ be a complete, irreducible subcurve of $X$. We will say that $E$ is a destabilizing component for $X$ if $E$ is smooth, rational, and meets the other components of $X$ in exactly two points; (similarly) a connected subcurve of $X$ all of whose irreducible components are destabilizing for $X$, will be called a destabilizing chain. Finally, we will denote by $D_{X}$ the destabilizing subcurve of $X$, that is, $D_{X}$ is defined to be the union of all destabilizing components of $X$. Let $q$ be a node of $X$; we will say that $q$ is a stable node if $q$ does not lie on any destabilizing component of $X$. Let $\sigma: X^{\prime} \rightarrow X$ be a morphism between two DM-semistable curves; we say that the node $q$ of $X$ is destabilized in $X^{\prime}$ if $\sigma^{-1}(q)$ is a destabilizing chain for $X^{\prime}$.

By the word "family" we will always mean a proper, flat morphism, whose fibers are curves. A one-parameter deformation of the curve $X$ will be a family $\mathscr{Z} \rightarrow\left(B, b_{0}\right)$, where $B$ is a smooth, one-dimensional scheme, $b_{0}$ a closed point of $B$, and $X$ the fiber over $b_{0}$; as is customary, the fiber over the point $b \in B$ will be denoted by $X_{b}$. A one-parameter deformation of a given curve will be denoted by a script capital letter and the deformed curve by the same letter, in roman style.

A polarized deformation $(\mathscr{Z}, \mathscr{L})$ of the couple $(X, L)$ ( $X$ a curve, $L$ a line bundle on it) is a deformation of $X$ together with a line bundle $\mathscr{L}$ on the total space, with the property that the restriction of $\mathscr{L}$ to $X$ is isomorphic to $L$. We will often use the notation $(\mathscr{X}, \mathscr{L}) \rightarrow B$ to denote a polarized family over $B$.

If $A$ and $B$ are schemes over $S$, we will denote by $p_{A}$ the natural projection

$$
p_{A}: A \times_{S} B \longrightarrow A \text {. }
$$

Let $d$ be an integer. The symbol $\underline{d}$ will always denote an ordered $n$-uple of integers $\left(d_{1}, \ldots, d_{n}\right)$ such that $\sum d_{i}=d$. Given two integers $n$ and $m$, we will denote by $(n, m)$ the greatest common divisor of $n$ and $m$.

If $V$ is a vector space, we will denote by $\mathbb{P}(V)$ the set of dimension 1 quotients of $V$.

1.2. Background in Geometric Invariant Theory. Geometric Invariant Theory provides a solution for the problem of defining and constructing quotients in the realm of algebraic geometry. We will start with an introductory analysis and 
afterwards we will summarize the fundamental results in a theorem. References for everything that follows are [GIT] and [N].

We will be interested in the special case of a projective scheme $Z$ endowed with an action of a reductive algebraic group $G$ (we may think of $G$ as equal to $S L(r+1)$ ). Let us consider an embedding of $Z$ in some projective space $\mathbb{P}(V)$, so that $Z=$ Proj $R, R$ some graded ring, finitely generated over $k$. If the action of $G$ on $Z$ can be lifted to a linear action on $V$, one says that $G$ acts linearly with respect to such an embedding. If that happens, $G$ will of course act on $R$; the subring of elements of $R$ which are invariant under the action of $G$ is denoted by $R^{G}$. It is a foundational theorem of Geometric Invariant Theory that, if $G$ is reductive, then $R^{G}$ is a graded algebra, finitely generated over $k$.

Now, let us consider the inclusion $R^{G} \subset R$ and the associated rational map

$$
\pi: \operatorname{Proj} R=Z \rightarrow Q:=\operatorname{Proj} R^{G} \text {. }
$$

This leads us to consider a special $G$-invariant open subset of $Z$ :

$$
Z_{R}^{S S}:=\left\{z \in Z \text { s.t. } \exists \text { a homogeneous nonconstant } f \in R^{G} \text { with } f(z) \neq 0\right\},
$$

that is, $Z_{R}^{S S}$ is precisely the locus where $\pi$ is regular. Notice that, although it is natural to view $Q$ as the quotient of $Z_{R}^{S S}$ modulo $G$, the fibers of $\pi$ may very well fail to be equal to the orbits of $G$; just consider the (common) case when there are nonclosed orbits. In other words, the closed points of $Q$ will not in general be in one-to-one correspondence with the orbits of $G$. This should motivate the following definition:

$Z_{R}^{S}:=\left\{z \in Z_{R}^{S S}\right.$ s.t. $\overline{O_{G}(z)} \cap Z_{R}^{S S}=O_{G}(z)$ and $\operatorname{dim} O_{G}(z)$ is

maximum among the dimensions of all $G$-orbits in $\left.Z_{R}^{S S}\right\}$.

The expectation is that the fibers of the restriction of $\pi$ to $Z_{R}^{S}$ are equal to orbits.

We finally make the previous discussion precise by stating

Theorem (Fundamental Theorem of GIT). Let $G$ be a reductive group acting linearly on the projective scheme $Z=\operatorname{Proj} R$. Then $Q:=\operatorname{Proj} R^{G}$ is a projective scheme and the natural morphism

$$
\pi: Z_{R}^{S S} \longrightarrow Q
$$

satisfies the following properties:

(1) For every $x, y \in Z_{R}^{S S}, \pi(x)=\pi(y)$ if and only if $\overline{O_{G}(x)} \cap \overline{O_{G}(y)} \cap Z_{R}^{S S}$ $\neq \varnothing$.

(2) (Universality) If there exists a scheme $Q^{\prime}$ with a G-invariant morphism $\pi^{\prime}: Z_{R}^{S S} \longrightarrow Q^{\prime}$, then there exists a unique morphism $\rho: Q \longrightarrow Q^{\prime}$ such that $\pi^{\prime}=\rho \circ \pi$.

(3) For every $x, y \in Z_{R}^{S}, \pi(x)=\pi(y)$ if and only if $O_{G}(x)=O_{G}(y)$. 
The usual notation is as follows:

(a) The points in $Z_{R}^{S S}$ are called semistable (or $R$-semistable).

(b) The points in $Z_{R}^{S}$ are called stable.

(c) The nonsemistable points, that is, the points in $Z \backslash Z_{R}^{S S}$, are called (alas!) unstable.

(d) $Q$ is denoted by $Z_{R}^{S S} / Q .^{3}$

(e) A quotient which satisfies property (3), that is, $Z_{R}^{S S}=Z_{R}^{S}$, is called a geometric quotient.

Once the linearization is fixed, the $R$ is usually dropped from the notation and one writes $Z^{S S}$ and $Z^{S}$ instead of $Z_{R}^{S S}$ and $Z_{R}^{S}$. We want to stress that, if we change the linearization, that is, if we change the embedding of $Z$ and the lifting of the group action, we will in general get different sets of semistable and stable points.

An immediate consequence of the fact that $Z^{S S} / G$ is projective is the socalled semistable replacement property of Geometric Invariant Theory, which we will often use in the sequel.

1.2.1. Semistable replacement property of GIT. Let $B$ be a smooth, one-dimensional scheme, $b_{0}$ be a closed point of $B$, and $f$ be a morphism of $B \backslash\left\{b_{0}\right\}$ to $Z^{S S}$. Then there exists a ramified cover $B^{\prime}$ of $B$ and a morphism $f^{\prime}$ of $B^{\prime}$ to $Z^{S S}$ such that

(1) the diagram below is commutative:

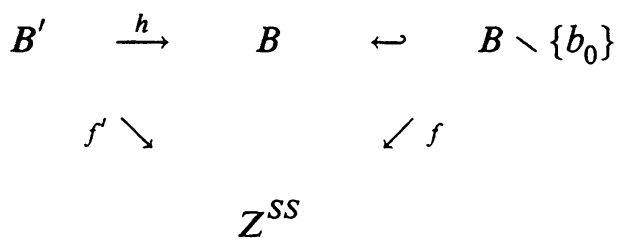

(2) there exists a point $b_{0}^{\prime}$ in $B^{\prime}$ with $h\left(b_{0}^{\prime}\right)=b_{0}$ and a morphism

$$
g: B^{\prime} \backslash\left\{b_{0}^{\prime}\right\} \longrightarrow G
$$

such that for every $b^{\prime} \in B^{\prime} \backslash\left\{b_{0}^{\prime}\right\}$ we have $f\left(h\left(b^{\prime}\right)\right)=g\left(b^{\prime}\right) \cdot f^{\prime}\left(b^{\prime}\right)$.

Moreover, $b_{0}^{\prime}$ can be chosen so that $f^{\prime}\left(b_{0}^{\prime}\right)$ is either stable, or with stabilizer of positive dimension.

We will conclude this summary by stating the so-called numerical criterion for stability, which has so far proven itself to be the most efficient tool available to test stability, and which we will use in the sequel. Roughly, such a theorem says that the study of the action of a reductive group can be reduced to the study of the action of its one-parameter subgroups.

From now till the end of this section we will work with a reductive group $G$ acting linearly on $\mathbb{P}(V)$ and with a closed $G$-invariant subscheme $Z$ of $\mathbb{P}(V)$.

\footnotetext{
${ }^{3}$ Sometimes the notation $Z_{R}^{S S} / / Q$ appears in the literature, to distinguish from the ordinary quotient. We will never use it.
} 
Recall that if $G_{m}$ denotes $k^{*}$ with multiplicative group structure and

$$
\lambda: G_{m} \longrightarrow G
$$

is a one-parameter subgroup of $G$, there exist a basis $\left\{v_{0}, \ldots, v_{r}\right\}$ for $V$ and integers $\left\{w_{0}, \ldots, w_{r}\right\}$ such that the action of $\lambda$ on $V$ is given by

$$
\lambda(t) \cdot v_{i}=t^{w_{i}} v_{i}
$$

for every $t$ in $G_{m}$ and $0 \leq i \leq r$. If $v=\sum a_{i} v_{i}, a_{i} \in k$, then those integers $n_{j}$ such that $a_{j}$ does not vanish are called the $\lambda$-weights of $v$.

Now let $z$ be a point in $Z$ and let $v_{z}$ be any vector in $V$ which is mapped to $z$ by the natural map $V \backslash\{0\} \longrightarrow \mathbb{P}(V)$. Having set the above notation, we can state the numerical criterion:

Theorem (Hilbert-Mumford Theorem). (1) $z \in Z$ is semistable $\Longleftrightarrow$ for every one-parameter subgroup $\lambda$ of $G$ the $\lambda$-weights of $v_{z}$ are not all positive.

(2) $z \in Z$ is stable $\Longleftrightarrow$ for every one-parameter subgroup $\lambda$ of $G v_{z}$ has both positive and negative $\lambda$-weights.

(3) $z \in Z$ is unstable $\Longleftrightarrow$ there exists a one-parameter subgroup $\lambda$ of $G$ such that the $\lambda$-weights of $v_{z}$ are all positive.

Of course the way we stated the theorem is redundant, as (1) and (3) are equivalent.

1.3. Stable curves in the sense of Deligne and Mumford. Let $M_{g}$ be the moduli space of smooth curves of genus $g ; M_{g}$ is a reduced, irreducible algebraic scheme whose closed points correspond to isomorphism classes of smooth curves of genus $g$. More precisely, $M_{g}$ is uniquely determined as the scheme which coarsely represents the contravariant functor $\mathscr{M}_{g}$ from schemes to sets defined as follows: for any scheme $S, \mathscr{M}_{g}(S)$ is the set of all proper and flat families of smooth curves of genus $g$ over $S$. If $f: S^{\prime} \rightarrow S$ is a morphism of schemes, $\mathscr{M}_{g}(f)$ is just given by taking fiber products.

We recall that to say that a scheme $F$ coarsely represents the functor $\mathscr{F}$ is to say that there exists a morphism of functors

$$
\Xi: \mathscr{F} \longrightarrow \mathscr{H} \text { om }(, F)
$$

such that:

(i) for every algebraically closed field $K$, the map

$$
\Xi(\operatorname{Spec} K): \mathscr{F}(\operatorname{Spec} K) \longrightarrow \mathscr{H} o m(\operatorname{Spec} K, F)
$$

is an isomorphism;

(ii) $F$ satisfies the following universal property: given a scheme $F^{\prime}$ and a morphism of functors

$$
\Xi^{\prime}: \mathscr{F} \longrightarrow \mathscr{H} \text { om }\left(, F^{\prime}\right)
$$

there exists a unique morphism

$$
\psi: F \longrightarrow F^{\prime}
$$


such that $\Xi^{\prime}=\Psi \cdot \Xi$ where for every $S$,

$$
\begin{aligned}
\Psi(S): \mathscr{H} \circ m(S, F) & \rightarrow \mathscr{H} \circ m\left(S, F^{\prime}\right) \\
f & \mapsto \psi \circ f .
\end{aligned}
$$

$M_{g}$ is not proper, in fact a family of smooth curves may very well have a singular limit. The problem of finding a meaningful compactification of it can be set in the following way:

(1) To find a class $\mathscr{C}$ of curves containing all smooth curves and such that

(i) $\mathscr{C}$ is big enough that every family whose general fiber is smooth (or in $\mathscr{C}$ ) is birational to one having all fibers in $\mathscr{C}$ and the same general fiber as the first family;

(ii) $\mathscr{C}$ is small enough to satisfy the separation property, that is, a family of curves in $\mathscr{C}$ should have a unique (up to isomorphism) limit in $\mathscr{C}$.

(2) To construct a projective scheme which is a moduli space for isomorphism classes of curves in $\mathscr{C}$.

The answer to the first part of the problem was provided by Deligne and Mumford, following ideas by Mayer and Mumford. The beauty of it is that precisely the "optimistic" try for $\mathscr{C}$ turns out to work. More precisely, one starts by including in $\mathscr{C}$ only curves with the simplest possible type of singularities, that is, ordinary double points (nodes). It is easy to see that this class cannot possibly satisfy any separation property; for example, one can just blow up a point of the total space of a one-parameter family of nodal curves to obtain a different family of nodal curves, and yet generically isomorphic to the first one. To get rid of this particular nonseparatedness phenomenon it is enough to consider the subclass of nodal curves having no smooth rational components, such as the ones arising from blowing-ups of the type we just described. This is easily made precise by the following definition:

Definition. A Deligne-Mumford stable curve is a reduced connected curve having only nodes as singularities and such that every smooth rational component meets the remaining components in at least three points.

The upshot is that Deligne-Mumford stable curves of fixed arithmetic genus have a moduli space which is a projective variety and contains $M_{g}$ as a dense subset; the usual notation for it is $\bar{M}_{g}$. As we promised, we will describe the Geometric Invariant Theory construction ${ }^{4}$ of such a compactification of $M_{g}$ in the following section. Much before such a construction was carried out, Deligne and Mumford proved that their stable curves satisfy both the separation and the properness property, which are the necessary requirements for a projective moduli space to exist. Their result can be stated as follows (cf. Lemma 1.12 in [DM]).

\footnotetext{
${ }^{4}$ It should be mentioned that there are other constructions of $\bar{M}_{g}$ which do not use Geometric Invariant Theory; see, for example, [Ko].
} 
1.3.1. Properness property. Let $\mathscr{Y}$ and $\mathscr{Z}$ be two families of Deligne and Mumford-stable curves over $\operatorname{Spec} \mathrm{R}$, where $\mathrm{R}$ is a discrete valuation ring.

Then, every isomorphism between the generic fibers of $\mathscr{Y}$ and $\mathscr{Z}$ extends to the whole of Spec R.

1.4. The GIT-construction of $\bar{M}_{g}$. To set up the GIT-situation, one has to use the existence of Grothendieck Hilbert schemes. We will briefly describe them in the special case that is necessary to our purpose.

Let us fix the following data: a projective space $\mathbb{P}^{r}$, integers $d \gg 0$ and $g \geq 3$ with $r=d-g$, and a polynomial $p(x)=d x-g+1$. Then there exists a projective scheme $\mathrm{Hilb}_{r}^{p(x)}$ whose closed points are in one-to-one correspondence with closed subschemes of $\mathbb{P}^{r}$ having Hilbert polynomial equal to $p(x)$. More precisely, $\mathrm{Hilb}_{r}^{p(x)}$ finely represents the contravariant functor $\mathscr{K}_{i l b_{r}^{p(x)}}$ from schemes to sets such that $\mathscr{H}_{i l b_{r}^{p(x)}}(S)$ is the set of proper and flat embedded families over $S$, whose fibers have Hilbert polynomial equal to $p(x)$. Here to say that a scheme $F$ represents the functor $\mathscr{F}$ is to say that $F$ coarsely represents $\mathscr{F}$ (see 1.3) plus the following condition:

The morphism of functors

$$
\Xi: \mathscr{F} \longrightarrow \mathscr{H} \text { om }(, F)
$$

is an isomorphism.

In our case, this is equivalent to the existence of a "universal" family over $\mathrm{Hilb}_{r}^{p(x)}$ together with a tautological polarization:

$$
\mathscr{L} \rightarrow \mathscr{X}_{r}^{p(x)} \stackrel{u}{\longrightarrow} \operatorname{Hilb}_{r}^{p(x)}
$$

such that for every $h$ in the Hilbert scheme, the fiber $X_{h}=u^{-1}(h)$ is isomorphic to the subscheme of $\mathbb{P}^{r}$ parametrized by $h$, and $L_{h}:=\mathscr{L}_{\mid X_{h}}$ is isomorphic to the line bundle giving the embedding of $X_{h}$. We will also use the somewhat inappropriate notation $h=\operatorname{hilb}\left(X_{h}, L_{h}\right)$, without specifying the basis giving the embedding and assuming that the embedding is complete; we will usually simply write $h=\operatorname{hilb}(X, L)$ or $h=\operatorname{hilb}(X)$, meaning $X \cong X_{h}$ and $L \cong L_{h}$. To say that $\Xi$ is an isomorphism is to say that, given any scheme $S$, every polarized family in $\mathscr{H} i l b_{r}^{p(x)}(S)$ is obtained in a unique way as the fiber product of the universal family described above.

We will now recall how to construct the Hilbert point of a given $X \subset \mathbb{P}^{r}$; that is, we will pointwise construct $\mathrm{Hilb}_{r}^{p(x)}$.

Let $L:=\mathscr{O}_{\mathbb{P}^{r}}(1) \otimes \mathscr{O}_{X}$; then, by a theorem of Serre, for $m$ large enough we have the exact sequence

$$
0 \longrightarrow H^{0}\left(\mathbb{P}^{r}, \mathscr{I}_{X}(m)\right) \longrightarrow H^{0}\left(\mathbb{P}^{r}, \mathscr{O}_{\mathbb{P}^{r}}(m)\right) \longrightarrow H^{0}\left(X, L^{m}\right) \longrightarrow 0
$$

where $\mathscr{I}_{X}$ is the ideal sheaf of $X$. Moreover, one can prove that there exists an integer $m^{\prime}$ such that for all $m \geq m^{\prime}$ and for all subschemes of $\mathbb{P}^{r}$ having $p(x)$ as Hilbert polynomial, the above sequence is exact and the degree- $m$ part of the ideal of $X$, that is, $H^{0}\left(\mathbb{P}^{r}, \mathcal{I}_{X}(m)\right)$, uniquely determines $X$. Hence we 
can associate to any such $X$ a point in the Grassmannian of $p(m)$-dimensional quotients of $S_{m}:=H^{0}\left(\mathbb{P}^{r}, \mathscr{O}_{\mathbb{P}^{r}}(m)\right)$, and such correspondence is injective.

Even a sketch of the proof that the set of all Hilbert points can be given the structure of a projective scheme satisfying the moduli property that we dicussed before would take too much room; as it is not really useful for our purposes, we just refer to Mumford's lectures [M1] for the complete construction. But the simple set-theoretic description that we just exhibited will be helpful to understand the Geometric Invariant Theory set-up.

Notice first of all that for any $m \geq m^{\prime}$ we have a different embedding of $\mathrm{Hilb}_{r}^{p(x)}$ in a Grassmannian, and hence in a projective space

$$
l_{m}: \operatorname{Hilb}_{r}^{p(x)} \hookrightarrow \mathbb{P}\left(\stackrel{p(m)}{\wedge} H^{0}\left(\mathbb{P}^{r}, \mathscr{O}_{\mathbb{P}^{\prime}}(m)\right)\right) .
$$

Now we have a natural linear action of the group $G=S L(r+1)$ on $\mathbb{P}\left(\stackrel{p(m)}{\wedge} S_{m}\right)$, hence any such $l_{m}$ determines a linearization of the action of $G$ on $\mathrm{Hilb}_{r}^{p(x)}$ (which is obviously $G$-invariant).

The goal is to construct $\bar{M}_{g}$ as the quotient of a suitable subscheme of Hilb $_{r}^{p(x)}$; for this reason it will be convenient to have a version of the HilbertMumford theorem which is specific to this set-up. $G$ acts naturally on $S_{m}$ and on $\wedge^{p(m)} S_{m}$, and so does the general linear group $G L(r+1)$. It turns out to be better to look at one-parameter subgroups of $G L(r+1)$ whose diagonal form is

$$
\lambda(t)=\operatorname{diag}\left(t^{w_{0}}, \ldots, t^{w_{r}}\right)
$$

where $w_{i} \in \mathbb{Z}$ and $0 \leq w_{0} \leq \cdots \leq w_{r}$. See [G], Section $0 . \mathrm{B}$, for a very clear explanation of how to make such a reduction and how to prove the statement below.

The Hilbert-Mumford criterion for Hilbert points is

Numerical criterion. Let $h=\operatorname{hilb}(X, L)$ be a closed point in $\mathrm{Hilb}_{r}^{p(x)}$; then $h$ is $S L(r+1)$-semistable (resp. stable) if and only if for every one-parameter subgroup $\lambda$ of $G L(r+1)$ as above there exist $p(m)$ monomials (in the basis diagonalizing $\lambda$ )

$$
\left\{M_{1}, \ldots, M_{p(m)}\right\} \in H^{0}\left(\mathbb{P}^{r}, \mathscr{O}_{\mathbb{P}^{r}}(m)\right)
$$

which restrict to a basis of $H^{0}\left(X, L^{m}\right)$ and such that

$$
\sum_{j=1}^{p(m)} w_{j}\left(M_{j}\right) \leq \frac{\sum_{0}^{r} w_{j}}{d-g+1} m p(m)
$$

$($ resp <).

Here by $w_{\lambda}\left(M_{i}\right)$ we denote the weight of $\lambda$ on $M_{i}$; that is, let $\left\{x_{0}, \ldots, x_{r}\right\}$ be a basis of $H^{0}\left(\mathbb{P}^{r}, \mathscr{O}_{\mathbb{P}^{\prime}}(1)\right)$ such that

$$
\lambda(t) \cdot x_{i}=t^{w_{i}} x_{i}, \quad 0 \leq w_{0} \leq \cdots \leq w_{r} ;
$$


then

$$
M=x_{0}^{m_{0}} \cdots \cdots x_{r}^{m_{r}} \Longrightarrow w_{\lambda}(M)=\sum m_{i} w_{i} \text {. }
$$

We will see applications of such a version of the Hilbert-Mumford Theorem in Sections 3.1 and 3.2.

As we said, we want to find a closed subscheme $K$ of $\left(\mathrm{Hilb}_{r}^{p(x)}\right)^{S S}$ such that $\bar{M}_{g}=K / G . K$ should satisfy the following requirements:

(1) All points of $K$ are $G$-stable, for some $m$.

(2) For every Deligne-Mumford stable curve $X$ there exists a unique orbit in $K$ parametrizing embeddings of $X$ in $\mathbb{P}^{r}$.

(3) All points of $K$ parametrize Deligne-Mumford stable curves.

The results that one uses at this point are

Theorems. Let $d \geq 20(g-1)$; then there exist infinitely many linearizations of the action of $S L(r+1)$ on $\mathrm{Hilb}_{r}^{p(x)}$ such that

(1) (Mumford-Gieseker Theorem) If $X \subset \mathbb{P}^{r}$ is a smooth, connected, nondegenerate curve of genus $g$ and degree $d$, then its Hilbert point is stable.

(2) (Gieseker Theorem) If $h \in \mathrm{Hilb}_{r}^{p(x)}$ is G-semistable, then all connected components of $X_{h}$ are Deligne-Mumford semistable curves; moreover, the embedding induced by $L_{h}$ is complete and $H^{1}\left(X_{h}, L_{h}\right)=0$.

See $[\mathrm{MM}]$ or $[\mathrm{G}]$ for (1) and [G] for (2).

Now, let us consider $d=n(2 g-2)$ and let us fix once and for all an $m$ for which these two theorems hold. Then we can consider the following subset of $\left(\mathrm{Hilb}_{r}^{p(x)}\right)^{S S}$ :

$$
\begin{aligned}
K=\left\{h \in \operatorname{Hilb}_{r}^{p(x)}\right. & \text { such that } h \text { is semistable, } \\
& \left.X_{h} \text { is connected, and } L_{h} \cong \omega_{X_{h}}^{\otimes n}\right\},
\end{aligned}
$$

$\omega_{X}$ being the dualizing sheaf of $X . K$ is clearly $G$-invariant and, by Gieseker's Theorem, it only parametrizes DM-stable curves; in fact, for any $n \geq 3, \omega_{X}^{\otimes n}$ is very ample if $X$ is DM-stable and it contracts precisely the destabilizing components of a DM-semistable curve. Moreover, $K$ only consists of $G$-stable points, and can be shown to be a closed subscheme of $\left(\mathrm{Hilb}_{r}^{p(x)}\right)^{S S}$. The final thing one needs to prove to conclude that $K / G$ is the moduli space of stable curves, is that the $n$ th-canonical projective model of every stable curve is in $K$, that is, it has semistable Hilbert point. The key point in the proof of this is precisely the properness property of stable curves that we stated at the end of the previous section.

All the details of this construction of $\bar{M}_{g}$ are in [G1].

\section{Construction of $\overline{P_{d, g}}$}

2.1. Existence of $\overline{P_{d, g}}$. From now on, we will assume $d \geq 20(g-1), g \geq 3$, and $r=d-g$. We will use the notation introduced in 1.4 and we will consider 
the Hilbert scheme $\mathrm{Hilb}_{r}^{p(x)}$ parametrizing closed subschemes of $\mathbb{P}^{r}$, having Hilbert polynomial $p(x)=d x-g+1$.

We will fix once and for all an $m$ for which Theorems (1) and (2) in 1.4 hold, and we will focus our attention on $\left(\mathrm{Hilb}_{r}^{p(x)}\right)_{m}^{S S}$, the subset of points of $\mathrm{Hilb}_{r}^{p(x)}$ which are semistable under the linearization of the action of $G$ induced by $l_{m}$. From now on we will simply say that a point in $\mathrm{Hilb}_{r}^{p(x)}$ is semistable (unstable, stable) if it belongs to $\left(\mathrm{Hilb}_{r}^{p(x)}\right)_{m}^{S S}$, and we will not mention the integer $m$ any longer. If $X \subset \mathbb{P}^{r}$ is a curve whose Hilbert polynomial is $p(x)$, and if $h$ is its Hilbert point, we will say that $X$ is $G$-semistable if $h$ is semistable for the action of $G$.

Let us define the set $H_{d} \subset\left(\operatorname{Hilb}_{r}^{p(x)}\right)^{S S}$ :

$$
H_{d}=\left\{h \in \operatorname{Hilb}_{r}^{p(x)}: X_{h} \text { is connected and } h \text { is semistable }\right\} .
$$

Notice that by Gieseker's Theorem, the points of $\left(\mathrm{Hilb}_{r}^{p(x)}\right)^{S S}$ correspond to reduced curves; therefore, the condition of being connected can be equivalently expressed by saying that $h^{0}\left(X_{h}, \mathscr{O}_{X_{h}}\right)=1$ or by the usual condition on the underlying topological space. The first condition is open, by upper-semicontinuity, the second one is closed. Hence we can conclude that $H_{d}$ is both open and closed in $\left(\mathrm{Hilb}_{r}^{p(x)}\right)^{S S}$, in other words, $H_{d}$ is a union of connected components of the locus of semistable points of $\mathrm{Hilb}_{r}^{p(x)}$.

Let

$$
u_{d}: \mathscr{X}_{d} \longrightarrow H_{d}
$$

be the restriction of the universal curve to it. By Mumford-Gieseker's Theorem, $H_{d}$ is nonempty, in fact for every $[X]$ in $M_{g}$, and for all $L$ in $\operatorname{Pic}_{X}^{d}$, the Hilbert point $\operatorname{hilb}(X, L)$ is in $H_{d}$.

By the fundamental theorem of Geometric Invariant Theory, there exists a quotient of $H_{d}$ by $G$,

$$
\pi_{d}: H_{d} \longrightarrow H_{d} / G
$$

where $\pi$ is a surjective, $G$-invariant morphism and $H_{d} / G$ is a projective scheme.

The next step is that $H_{d}$ maps naturally to $\bar{M}_{g}$. To show this, we need to construct a "canonical" family of stable curves over $H_{d}$. Recall that the fibers of the universal family $u_{d}: \mathscr{Z}_{d} \rightarrow H_{d}$ are DM-semistable curves; our claim will therefore follow from the following general fact:

If $u: \mathscr{Z} \rightarrow B$ is any family of DM-semistable curves, there exists a canonical, commutative diagram

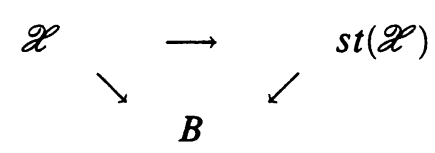

where

$$
\operatorname{st}(u): \operatorname{st}(\mathscr{Z}) \rightarrow B
$$


is a family of DM-stable curves such that for every $b$ in $B, s t(u)^{-1}(b)$ is the stable model of $X_{b}$, i.e., the DM-stable curve obtained by contracting to an ordinary double point every destabilizing rational component of $X_{b}$.

We will call the family $s t(u): s t(\mathscr{Z}) \rightarrow B$ the stable model or stable reduction of $\mathscr{Z}$ over $B$. To construct such a stable model, consider the dualizing sheaf $\omega=\omega_{\mathscr{Z} / B}$ of the family $u: \mathscr{Z} \rightarrow B ; u_{*}\left(\omega^{\otimes 3}\right)$ is locally free on $H_{d}$ and there is a diagram

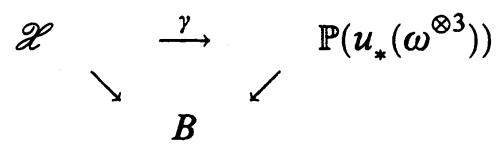

such that the restriction of $\gamma$ to any fiber $X_{b}$ maps it to a DM-stable curve in $\mathbb{P}^{5 g-6}$. This clearly follows from the fact that $\omega^{\otimes 3}$ restricts to a very ample line bundle on each DM-stable fiber, whereas its restriction to any destabilizing component of a DM-semistable fiber has degree 0 . Therefore, there exists a unique morphism $\psi_{d}: H_{d} \rightarrow \bar{M}_{g}$ such that $\psi_{d}(h)=\left[s t\left(X_{h}\right)\right]$.

We now want to show that this map is surjective. This is a simple consequence of the semistable replacement property of Geometric Invariant Theory (cf. 1.2) and of the properness property of DM-stable curves (cf. 1.3).

Lemma 2.1. Let $[X] \in \bar{M}_{g}$; then there exists a point $h \in H_{d}$ such that the stable model of $X_{h}$ is $X$.

Proof. Let us consider a one-parameter deformation of $X$ to a smooth curve, that is, a family

$$
f: \mathscr{X} \longrightarrow\left(B, b_{0}\right)
$$

where $B$ is a smooth curve and such that the fiber of $\mathscr{X}$ over $b \in B$ is nonsingular for all $b \neq b_{0}$ while the fiber over $b_{0}$ is isomorphic to $X$. If $\mathscr{L}$ is any line bundle on $\mathscr{X}$ having degree $d$ on the fibers, after shrinking $B$ around $b_{0}$ we can choose a frame for $f_{*} \mathscr{L}$; this will give a map $\gamma: B \rightarrow$ Hilb $_{r}^{p(x)}$ with the property that $\gamma\left(B \backslash\left\{b_{0}\right\}\right) \subset H_{d}$ (recall that the Hilbert point of a smooth, projective, linearly normal curve of degree $d$ is stable). By the semistable replacement property of Geometric Invariant Theory, up to taking a ramified cover of $\left(B, b_{0}\right)$, we can assume that there is a morphism $\bar{\gamma}: B \rightarrow H_{d}$ whose restriction to $B \backslash\left\{b_{0}\right\}$ is congruent to $\gamma$ modulo $G$.

Now we want to use the properness property to show that $\bar{\gamma}\left(b_{0}\right)$ corresponds to the Hilbert point of a curve having $X$ as stable model.

Let $\mathscr{Y}$ be the family of DM-semistable curves on $B$ obtained by pulling back $\mathscr{Z}_{d}$ to $B$ via $\bar{\gamma}$ and let $Y$ be its fiber over $b_{0} . \mathscr{Y}$ and $\mathscr{Z}$ are isomorphic away from their fibers over $b_{0}$; if $s t(\mathscr{Y}) \rightarrow B$ is the stable reduction of $\mathscr{Y}$, then $s t(\mathscr{Y})$ and $\mathscr{Z}$ are also isomorphic away from their fibers over $b_{0}$. By the properness property, such isomorphism extends, hence we can conclude that st $(Y) \cong X$.

Obviously, $\psi_{d}$ is constant along the orbits of $G$, hence, by the universal property of the GIT-quotient, it factors through the quotient morphism $\pi_{d}$ in a unique way. To conclude, we can state the main result of this section: 
Theorem 2.1. (1) There exists a projective scheme $\overline{P_{d, g}}$ with a natural (proper), surjective morphism $\phi_{d}$ onto $\bar{M}_{g}$.

(2) For all $[X] \in M_{g}^{0}$,

$$
\phi_{d}^{-1}([X]) \cong \operatorname{Pic}_{X}^{d}
$$

moreover

$$
\phi_{d}^{-1}\left(M_{g}^{0}\right) \cong P_{d, g} .
$$

Proof. To prove (1), we take

$$
\overline{P_{d, g}}:=H_{d} / G ;
$$

then the first part of the statement has already been proved. Note that, in fact, we have a canonical, commutative diagram:

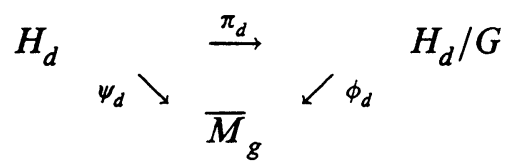

Now we will prove (2). If $[X] \in M_{g}^{0}$, we claim that $\phi_{d}^{-1}([X]) \cong \operatorname{Pic}_{X}^{d}$. There are two ways to prove this: one way is to just use the universal property of $\mathrm{Pic}_{X}^{d}$, the fine moduli space of line bundles of degree $d$ on $X$. In other words, we can use the fact that such a moduli space already exists as the (unique up to isomorphism) scheme which finely represents (cf. Sections 1.3 and 1.4) the contravariant functor $\mathscr{P} i c_{X}^{d}$ from the category of schemes to the category of sets. Here for any scheme $S, \mathscr{P} i c_{X}^{d}(S)$ is the set of isomorphism classes of line bundles over $X \times{ }_{k} S$ having relative degree $d$, modulo pull-backs of line bundles on $S$.

In fact, $\forall[X] \in \bar{M}_{g}$, let us denote the set of GIT-semistable projective models of $X$ by

$$
M_{X}=\left\{h \in H_{d}: X_{h} \cong X\right\} .
$$

If $X$ is smooth and has no nontrivial automorphisms, we can describe $M_{X}$ completely:

$$
M_{X}=\left\{\operatorname{hilb}(X, L), \forall L \in \operatorname{Pic}_{X}^{d}\right\}=\psi_{d}^{-1}([X]),
$$

where the last equality follows from Gieseker's theorem. $M_{X}$ is closed in $H_{d}$ and its points are all stable under the action of $G$.

At this stage, it is clear that $\phi_{d}^{-1}([X])$ is pointwise isomorphic to $\operatorname{Pic}_{X}^{d}$. Notice also that if $X$ has automorphisms, a point in $M_{X}$ determines the line bundle $L$ only up to automorphisms of $X$.

Now, by the universal property of the Hilbert scheme, for any family of line bundles of degree $d$ on $X$, parametrized by some base scheme $S$, we can construct a unique morphism

$$
S \rightarrow M_{X} / G=\phi_{d}^{-1}([X]) .
$$


In other words, there is a morphism of functors

$$
\Xi: \mathscr{P} i c_{X}^{d} \longrightarrow \mathscr{H} \text { om }\left(, \phi_{d}^{-1}([X])\right) .
$$

Therefore, by the universal property of $\mathrm{Pic}_{X}^{d}$, there correspondingly exists a unique morphism of schemes

$$
\xi: \operatorname{Pic}_{X}^{d} \longrightarrow M_{X} / G
$$

through which $\Xi$ is factorized.

To construct the inverse of $\xi$, first consider the morphism from $M_{X}$ to Pic $_{X}^{d}$ associated to the natural, polarized family over $M_{X}$; such a morphism is $G$-invariant, hence it can be descended to the quotient, providing the required

$$
\xi^{-1}: M_{X} / G \longrightarrow \operatorname{Pic}_{X}^{d}
$$

This concludes the proof of our statement.

Another, more interesting, approach is to directly prove that $M_{X} / G$ is the degree $d$ Picard variety, by showing that it satisfies the required moduli properties. By the above discussion, what still needs to be proved is the existence of a Poincaré line bundle over $M_{X} / G \times X$. We will now construct such a bundle, by using the tautological line bundle over the universal family over the Hilbert scheme. Our method can be viewed as a special case of a more general construction due to Maruyama (see [Ma]).

The restriction of $\mathscr{X}_{d}$ to $M_{X}$ is $M_{X} \times_{k} X$; let us denote by $\mathscr{L}$ the restriction of the tautological polarization to it.

We have the diagram:

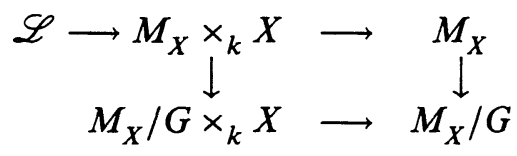

We cannot simply descend $\mathscr{L}$ to a line bundle over $M_{X} / G \times_{k} X$, because the stabilizers of the points of $M_{X}$ (all of which are isomorphic to the cyclic group of order $\left.r+1, \mu_{r+1}\right)$ may act nontrivially on the restriction of $\mathscr{L}$ to the fibers of

$$
M_{X} \times_{k} X \longrightarrow M_{X}
$$

But now, given a tautological polarization $\mathscr{L}$, we can obtain another one by taking

$$
\mathscr{L} \otimes p_{M_{X}}^{*} F
$$

for any line bundle $F$ on $M_{X}$. What we need to do then, is to find a line bundle $F$ endowed with an action of $G$ which kills the action of $\mu_{r+1}$ on $\mathscr{L}$.

We define

$$
F=\operatorname{det}\left(p_{M_{X}}(\mathscr{L})\right) \otimes \operatorname{det}\left(p_{M_{X}}\left(\mathscr{L} \otimes p_{X}^{*} \mathscr{O}_{X}(-p)\right)\right)^{-1}
$$


where $p$ is a point of $X$. It is clear that the action of $\mu_{r+1}$ on

$$
\mathscr{L}^{\prime}:=\mathscr{L} \otimes p_{M_{X}}^{*} F
$$

is trivial and that $\mathscr{L}^{\prime}$ descends to a line bundle on $M_{X} / G \times_{k} X$ which satisfies the defining property for a Poincaré bundle.

Finally, to prove that $\phi_{d}^{-1}\left(M_{g}^{0}\right)$ is isomorphic to $P_{d, g}$ we have to show that $\phi_{d}^{-1}\left(M_{g}^{0}\right)$ coarsely represents the degree $d$ Picard functor $\mathscr{P}_{d, g}$ relative to the universal curve

$$
\mathscr{C}_{g} \longrightarrow M_{g}^{0}
$$

This is to say (cf. 1.3) that there exists a morphism of functors

$$
\Xi: \mathscr{P}_{d, g} \longrightarrow \mathscr{H} \text { om }\left(, \phi_{d}^{-1}\left(M_{g}^{0}\right)\right)
$$

such that:

(i) for every algebraically closed field $K$, the map

$$
\Xi(\operatorname{Spec} K): \mathscr{P}_{d, g}(\operatorname{Spec} K) \longrightarrow \mathscr{H} o m\left(\operatorname{Spec} K, \phi_{d}^{-1}\left(M_{g}^{0}\right)\right)
$$

is an isomorphism;

(ii) given a scheme $P$ and a morphism of functors

$$
\Gamma: \mathscr{P}_{d, g} \longrightarrow \mathscr{H} \text { om }(, P)
$$

there exists a unique morphism

$$
\psi: \phi_{d}^{-1}\left(M_{g}^{0}\right) \longrightarrow P
$$

such that $\Gamma=\Psi \cdot \Xi$.

Let us start by constructing $\Xi$. Let $\mathscr{F} \in \mathscr{P}_{d, g}(S)$; then $\mathscr{F}$ is a family of smooth curves of genus $g$ over $S$, with a polarization $\mathscr{L}$ of relative degree $d$ :

$$
\mathscr{L} \longrightarrow \mathscr{C} \stackrel{f}{\longrightarrow} S
$$

$f_{*} \mathscr{L}$ is locally free, hence there exists an open covering $S=\cup U_{i}$ such that for every $i$ we are given a trivialization of $f_{*} \mathscr{L}_{\mid U_{i}}$ so that there exists a morphism $f_{i}$ of $U_{i}$ to $\left(H_{d}\right)^{\text {stable }}$, given by $f_{i}(u):=\operatorname{hilb}\left(C_{u}, L_{u}\right)$. If we denote by $\bar{f}_{i}$ the composition of $f_{i}$ with the quotient map, it is clear that the $\bar{f}_{i}$ can be glued to a map, $\Xi(S)(\mathscr{F})$, from $S$ to $\phi_{d}^{-1}\left(M_{g}^{0}\right)$, which does not depend on the choice of the trivialization.

Now, the fact that $\Xi(\operatorname{Spec} K)$ is bijective follows immediately from the fact that the restriction of $\pi_{d}$ to

$$
H_{d}^{0}=\left\{h \in H_{d} \text { s.t. }\left[X_{h}\right] \in M_{g}^{0}\right\}
$$

is a geometric quotient. ${ }^{5}$

\footnotetext{
${ }^{5}$ Recall that a GIT- quotient $\pi: H \longrightarrow H / G$ is called geometric if every fiber of $\pi$ is equal to a $G$-orbit.
} 
Finally, to prove (ii), we consider the image under the map

$$
\left.\Gamma\left(H_{d}^{0}\right): \mathscr{P}_{d, g}\left(H_{d}^{0}\right) \longrightarrow \mathscr{H} \circ H_{d}^{0}, P\right)
$$

of the restriction of the universal family to $H_{d}^{0}$. This will be a $G$-invariant morphism

$$
\psi^{\prime}: H_{d}^{0} \longrightarrow P
$$

that can be factored through the quotient map $\pi_{d}$, that is, we obtained a morphism

$$
\psi: \phi_{d}^{-1}\left(M_{g}^{0}\right) \longrightarrow P
$$

which is what we wanted.

We will denote

$$
\overline{P_{d, X}}:=\phi_{d}^{-1}(X)
$$

2.2. Local geometry of $H_{d}$. We have the following result.

Lemma 2.2. (1) $H_{d}$ is nonsingular.

(2) Every connected component of $H_{d}$ has dimension $r(r+2)+4 g-3$.

Proof. $H_{d}$ is an open subset of $\mathrm{Hilb}_{r}^{p(x)}$. Recall that a lower bound for the dimension of any irreducible component of $\mathrm{Hilb}_{r}^{p(x)}$ is given by the number

$$
r(r+2)+4 g-3
$$

We will prove the lemma by showing that for all $h \in H_{d}$, the dimension of the tangent space of $H_{d}$ at $h$ is equal to $r(r+2)+4 g-3$. The essential point will be to observe that, since $h$ is semistable for the action of $G$, the corresponding subscheme of $\mathbb{P}^{r}, X_{h}$, is a nonspecial curve. Let us denote $X_{h}$ by $X, X$ will be a nodal curve embedded in $\mathbb{P}^{r}$ by $L$; let $\mathscr{I}_{X}$ be its ideal sheaf. Let us denote by $\mathscr{N}_{X / \mathbb{P}^{r}}$ the corresponding normal sheaf, that is,

$$
\mathscr{N}_{X / \mathbb{P}^{\prime}}:=\operatorname{Hom}_{\mathscr{O}_{X}}\left(\mathscr{I}_{X} / \mathscr{F}_{X}^{2}, \mathscr{O}_{X}\right) \text {. }
$$

Then we have the well-known identification

$$
T_{h} H_{d}=H^{0}\left(X, \mathscr{N}_{X / \mathbb{P}^{r}}\right)
$$

Since $X$ is locally a complete intersection, $\mathscr{N}_{X / \mathbb{P}^{\prime}}$ is locally free and, by applying the Riemann-Roch Theorem to it, we get

$$
\chi\left(\mathscr{N}_{X / \mathbb{P}^{r}}\right)=r(r+2)+4 g-3
$$

Therefore, to prove the lemma, we need to show that $h^{1}\left(X, \mathscr{N}_{X / \mathbb{P}^{\prime}}\right)=0$. 
There is the following standard exact sequence

$$
0 \rightarrow \mathscr{T}_{X} \rightarrow \mathscr{T}_{\mathbb{P}^{P}} \otimes \mathscr{O}_{X} \rightarrow \mathscr{N}_{X / \mathbb{P}^{r}} \rightarrow T_{X}^{1} \rightarrow 0
$$

(cf. [LS]) where $\mathscr{T}_{X}$ is the tangent sheaf of $X, \mathscr{T}^{\text {P }}$ is the tangent bundle of $\mathbb{P}^{r}$, and

$$
T_{X}^{1}=\operatorname{Ext}_{\mathscr{\theta}_{X}}^{1}\left(\Omega_{X}, \mathscr{O}_{X}\right) .
$$

Since $T_{X}^{1}$ is a skyscraper sheaf supported at the singular locus of $X$, we get a surjection

$$
H^{1}\left(X, \mathscr{T}_{\mathbb{P}^{r}} \otimes \mathscr{O}_{X}\right) \rightarrow H^{1}\left(X, \mathscr{N}_{X / \mathbb{P}^{r}}\right) \rightarrow 0
$$

This, together with the Euler sequence for $\mathbb{P}^{r}$, yields

$$
h^{1}\left(X, \mathscr{N}_{X / \mathbb{P}^{r}}\right) \leq h^{1}\left(X, \oplus_{1}^{r+1} \mathscr{O}_{X}(1)\right)=(r+1) h^{1}\left(X, \mathscr{O}_{X}(1)\right)=0
$$

where the last equality follows from Gieseker's Theorem (for the details, see [G], Proposition 1.0.8).

Corollary 2.1. The scheme $\overline{P_{d, g}}$ is reduced and Cohen-Maculay.

Proof. The fact that $\overline{P_{d, g}}$ is Cohen-Macaulay follows from the fact that $H_{d}$ is regular, by results by Hochster and Roberts [HR] about the GIT-quotient of a smooth variety.

Remark. We will show later (Lemma 6.2) that $H_{d}$ is actually irreducible.

The previous lemma is a special case of a more general result that we will now describe and that will also be useful. Let $X$ be a nodal connected curve in $\mathbb{P}^{r}$ and let $I$ be a subset of the nodes of $X$. Then there exists a locally closed subscheme $\operatorname{Hilb}_{X}(I)$ of $\operatorname{Hilb}_{r}^{p(x)}$ such that $\operatorname{Hilb}_{X}(I)$ parametrizes (flat) deformations of $X$ in $\mathbb{P}^{r}$ which are locally trivial at all the nodes contained in $I$ (cf. [LS] and [Sn]).

The result we need is the following:

Lemma 2.3. Let $h=\operatorname{hilb}(X)$ be in $H_{d}$, and let $I$ be any subset of the nodes of $X$. Then $I$ is flatly smoothable in $\mathbb{P}^{r} ;$ in other words, $\operatorname{Hilb}_{X}(I)$ is smooth at $h$ and it has dimension $r(r+2)+4 g-3-|I|$.

Proof. The proof is really a straightforward generalization of the proof of Lemma 2.2. By standard deformation theory the tangent space to $\operatorname{Hilb}_{X}(I)$ at $h$ is identified with $H^{0}\left(X, \mathscr{N}_{X / \mathbb{P}}^{I}\right)$ and $\operatorname{Hilb}_{X}(I)$ is smooth at $h$ if and only if $H^{1}\left(X, \mathscr{N}_{X / \mathbb{P}^{\prime}}^{I}\right)=0$; here $\mathscr{N}_{X / \mathbb{P}^{\prime}}^{I}$ is defined by the exact sequence

$$
0 \rightarrow \mathscr{N}_{X / \mathbb{P}^{\prime}}^{I} \rightarrow \mathscr{N}_{X / \mathbb{P}^{\prime}} \rightarrow T_{\left.X\right|_{I}}^{1} \rightarrow 0
$$

$\left(T_{\left.X\right|_{I}}^{1}\right.$ denotes the restriction of $T_{X}^{1}$ to $\left.I\right)$. 
In particular, notice that

$$
\mathscr{N}_{X / \mathbb{P}^{P}}^{\text {sing } X}=\operatorname{Ker}\left\{\mathscr{N}_{X / \mathbb{P}^{\prime}} \rightarrow T_{X}^{1}\right\}=\operatorname{Im}\left\{\mathscr{T}_{\mathbb{P}^{\prime}} \otimes \mathscr{O}_{X} \rightarrow \mathscr{N}_{X / \mathbb{P}^{\prime}}\right\}
$$

hence, from the proof of the previous lemma,

$$
H^{1}\left(X, \mathscr{N}_{X / \mathbb{P}^{\prime}}^{\text {sing } X}\right)=0 .
$$

Now, we have an exact sequence

$$
0 \rightarrow \mathscr{N}_{X / \mathbb{P}^{\prime}}^{\text {singX }} \rightarrow \mathscr{N}_{X / \mathbb{P}^{\prime}}^{I} \rightarrow T_{\left.X\right|_{\text {sing } X \backslash I} ^{1}}^{1} \rightarrow 0
$$

hence

$$
H^{1}\left(X, \mathscr{N}_{X / \mathbb{P}^{\prime}}^{I}\right)=0
$$

and the lemma is proved.

\section{What IS THE MULTIDEGREe OF A GIT-SEMistable CURVE?}

3.1. The Basic Inequality. We will now state the theorem which is the key tool in the analysis of $\overline{P_{d, g}}$ and $\overline{P_{d, X}}$. In this section, we will only prove a part of it; the remaining half will be proved in Section 6.1. The crucial observation is that a curve in $\mathbb{P}^{r}$ should have better chances to have semistable Hilbert point if its multidegree is proportional to its multigenus. This is made precise by the statement below.

Theorem (Basic Inequality). Let $X$ be a connected, nondegenerate, nodal, curve in $\mathbb{P}^{r}$ having genus $g$ and degree $d$. For $\operatorname{hilb}(X)$ to be semistable it is necessary and sufficient that, for any complete subcurve $Y$ of $X$, the degree of $Y$ satisfies the following inequality:

$$
\left|d_{Y}-\frac{d}{(g-1)}\left(g_{Y}-1+k_{Y} / 2\right)\right| \leq k_{Y} / 2
$$

where $k_{Y}=|Y \cap \overline{X \backslash Y}|$.

We will prove the two directions of the statement separately. The necessary condition is restated and proved below, as Proposition 3.1. The sufficient condition will be Proposition 6.1 in a following section. The proposition below determines a bounded domain in which the multidegree of a GIT-semistable curve $X$ can vary. We remark that such a first approximation is a very good one, for example, it will imply that the abstract curve $X$, which by Gieseker's Theorem is DM-semistable, can only have destabilizing chains of length 1 . In particular, we have the striking fact that, given any DM-stable curve $W$, there are only finitely many abstract curves having $W$ as stable model and admitting some GIT-semistable embedding of degree $d$ in $\mathbb{P}^{r}$.

To prove the necessary condition part we will follow the methods of Gieseker.

Proposition 3.1 (Basic Inequality: necessary condition). Let $X \subset \mathbb{P}^{r}$ be a projective curve such that $h=\operatorname{hilb}(X) \in H_{d}$, and let $Y \subset \mathbb{P}^{r}$ be any complete subcurve 
of $X$ having arithmetic genus $g_{Y}$ and intersecting the remaining components of $X$ in $k$ points. Then the degree of $Y$ satisfies the following inequality:

$$
\left|d_{Y}-\frac{d}{(g-1)}\left(g_{Y}-1+k / 2\right)\right| \leq k / 2 \text {. }
$$

Proof. Let $L \in \mathrm{Pic}_{X}^{\frac{d}{X}}$ be the very ample line bundle $\mathscr{O}_{\mathbb{P}^{P}}(1) \otimes \mathscr{O}_{X}$; we are assuming that $\operatorname{hilb}(X, L)=h$ is semistable under the group action on Hilb ${ }_{r}^{p(x)}$. Let $Y$ be any complete subcurve of $X$ and $Z=\overline{(X-Y)}$; then $k=|Y \cap Z|$. To show that $d_{Y}$ satisfies the Basic Inequality, we need to use the fact that, if $\operatorname{hilb}(X, L)$ is semistable, then for every subcurve $Y$ of $X$ we have

$$
\frac{h^{0}\left(Y, L_{Y}\right)}{h^{0}(X, L)} \geq \frac{d_{Y}+k / 2}{d}
$$

where $L_{Y}=L \otimes \mathscr{O}_{Y}$. We will refer to (G) as the Gieseker inequality.

The easy part of the proof consists in showing that the Gieseker inequality is equivalent to the Basic Inequality. Let us do that first.

By Gieseker's Theorem, $L$ embeds $X$ as a nonspecial curve, hence we have

$$
\frac{h^{0}\left(Y, L_{Y}\right)}{h^{0}(X, L)} \geq \frac{d_{Y}+k / 2}{d}
$$

$\Longleftrightarrow$

$$
\frac{d_{Y}-g_{Y}+1+h^{1}\left(Y, L_{Y}\right)}{d-g+1} \geq \frac{\dot{d}_{Y}+k / 2}{d}
$$

$\Longleftrightarrow$

$$
\frac{d}{g-1}\left(g_{Y}-1+k / 2\right) \leq d_{Y}+k / 2 \text {. }
$$

If we now apply the same argument to $Z$ we get

$$
\frac{d}{g-1}\left(g_{Z}-1+k / 2\right) \leq d_{Z}+k / 2 \text {; }
$$

and, since $d=d_{Y}+d_{Z}$ and $g=g_{Z}+g_{Y}+k-1$, we have

$$
\frac{d}{g-1}\left(g-g_{Y}-k / 2\right) \leq d-d_{Y}+k / 2
$$

$\Longleftrightarrow$

$$
\frac{d}{g-1}\left(g_{Y}-1+k / 2\right) \geq d_{Y}-k / 2 \text {. }
$$

Now, we have to show that $(G)$ is satisfied if $h$ is semistable. The proof of this fact is due to Gieseker; we will briefly describe it, since we need to more extensively analyze its consequences (a more detailed proof can be found in [G]). As it is standard in Geometric Invariant Theory, the method is to assume that $(G)$ is not satisfied and then to find a so-called "destabilizing oneparameter subgroup" for $h$, which highlights the fact that $(G)$ fails. More 
precisely, suppose that

$$
\frac{h^{0}\left(Y, L_{Y}\right)}{h^{0}(X, L)}<\frac{d_{Y}+k / 2}{d}
$$

then one can reach a contradiction by exhibiting a one-parameter subgroup $\lambda$ of $G$ such that $h$ is unstable with respect to $\lambda$.

Let us construct such a destabilizing $\lambda$. Let $V=H^{0}\left(\mathbb{P}^{r}, \mathscr{O}_{\mathbb{P}^{\prime}}(1)\right)$ and let

$$
U=\operatorname{Ker}\left\{H^{0}\left(\mathbb{P}^{r}, \mathscr{O}_{\mathbb{P}^{r}}(1)\right) \rightarrow H^{0}\left(Y, \mathscr{O}_{Y}(1)\right)\right\} \text {. }
$$

Then choose a basis $\left\{x_{0}, \ldots, x_{N}, \ldots, x_{r}\right\}$ relative to the filtration

$$
0 \subseteq U \subset V
$$

that is, $x_{i} \in U$ if $0 \leq i \leq N$; now we define

$$
\lambda(t) \cdot x_{i}= \begin{cases}x_{i}, & i \leq N, \\ t x_{i}, & i \geq N+1 .\end{cases}
$$

We will now show that, if $(\mathrm{G})$ is not satisfied, then $h$ is $\lambda$-unstable. By the numerical criterion for Hilbert points that we have stated in $1.4, h$ is unstable if for every $p(m)$-uple of monomials $\left\{M_{1}, \ldots, M_{p(m)}\right\}$ in the $\left\{x_{0}, \ldots, x_{r}\right\}$, which are independent over $X$, one has

$$
\sum_{j=1}^{p(m)} w_{j}\left(M_{j}\right)>\frac{\sum_{0}^{r} w_{j}}{d-g+1} m p(m) .
$$

Consider the filtration of $H^{0}\left(\mathbb{P}^{r}, \mathscr{O}_{\mathbb{P}^{r}}(m)\right)$ :

$$
0 \subseteq U^{m} \subseteq U^{m-1} V \subseteq \cdots \subseteq U^{m-i} V^{i} \subseteq \cdots \subset V^{m}=H^{0}\left(\mathbb{P}^{r}, \mathscr{O}_{\mathbb{P}^{r}}(m)\right)
$$

This of course induces a filtration $F$ of $H^{0}\left(X, L^{m}\right)$ via the (surjective) restriction map

$$
\rho_{m}: H^{0}\left(\mathbb{P}^{r}, \mathscr{O}_{\mathbb{P}^{r}}(m)\right) \rightarrow H^{0}\left(X, L^{m}\right) \text {. }
$$

Let $\left\{M_{1}, \ldots, M_{p(m)}\right\}$ be monomials which restrict to a basis of $H^{0}\left(X, L^{m}\right)$ relative to $F$. Clearly

$$
M_{j} \in U^{m-i} V^{i} \backslash U^{m-i+1} V^{i-1} \Longrightarrow w_{\lambda}\left(M_{j}\right)=i
$$

moreover, for any other $p(m)$-uple $\left\{M_{1}^{\prime}, \ldots, M_{p(m)}^{\prime}\right\}$, restricting to a basis of $H^{0}\left(X, L^{m}\right)$, we have

$$
\sum_{j=1}^{p(m)} w_{\lambda}\left(M_{j}\right) \leq \sum_{j=1}^{p(m)} w_{\lambda}\left(M_{j}^{\prime}\right)
$$

Therefore, to conclude that $h$ is unstable it is enough to prove that

$$
\sum_{j=1}^{p(m)} w_{\lambda}\left(M_{j}\right)>\frac{\sum_{0}^{r} w_{j}}{h^{0}(X, L)} m p(m) .
$$


Let $b_{i}=\operatorname{dim} \rho_{m}\left(U^{m-i} V^{i}\right)$ for $0 \leq i \leq m$; then

$$
T=\sum_{j=1}^{p(m)} w_{\lambda}\left(M_{j}\right)=m b_{m}-\sum_{i=0}^{m-1} b_{i}
$$

Let $f: Z^{\prime} \rightarrow Z$ be the normalization of $Z, L^{\prime}=f^{*} L, D=f^{-1}(Y \cap Z)$, and $g^{\prime}=g_{Z^{\prime}}$. We have

$$
b_{i} \leq h^{0}\left(Z^{\prime},\left(L^{\prime}\right)^{m}((i-m) D)\right)=d_{Z} m+(i-m) k-g^{\prime}+1+h_{i}^{1}
$$

where

$$
h_{i}^{1}=h^{1}\left(Z^{\prime},\left(L^{\prime}\right)^{m}((i-m) D)\right) \leq g^{\prime} ;
$$

the last inequality holds, provided that, on each connected component of $Z^{\prime}$ the line bundle in question has nonnegative degree. We will now suppose that this is the case and continue the proof under such an assumption. Since the proof of the general case is of no interest to us, we will simply refer to [G], p. 83. Then

$$
T \geq m b_{m}-\sum_{i=0}^{m-1}\left(m d_{z}+(i-m) k+1\right)=m^{2}\left(d-d_{Z}+k / 2\right)+O(m) .
$$

Finally, using the fact that $(G)$ fails together with the obvious relation

$$
h^{0}\left(Y, L_{Y}\right) \geq \sum_{0}^{r} w_{j}
$$

we have that there exists an integer $m^{\prime}$, which can be shown not to depend on $X$ and $L$, such that, for all $m>m^{\prime}$ we have

$$
T \geq m^{2}\left(d_{Y}+k / 2\right)+O(m)>m^{2} \frac{h^{0}\left(Y, L_{Y}\right)}{h^{0}(X, L)} d+O(m) \geq \frac{\sum_{0}^{r} w_{j}}{h^{0}(X, L)} m p(m) .
$$

This finishes the proof.

We will usually write the Basic Inequality as

$$
m_{Y} \leq d_{Y} \leq M_{Y}
$$

where

$$
m_{Y}=\frac{d}{(g-1)}\left(g_{Y}-1+k_{Y} / 2\right)-k_{Y} / 2 \text { and } M_{Y}=m_{Y}+k_{Y} .
$$

3.2. Behaviour at the extremes of the Basic Inequality. We now want to show that, as a corollary of the proof, if $d_{Y}$ is equal to one of the extremes of the Basic Inequality, and $X$ is DM-stable, then $h$ has no chance to be $G$-stable. As we will see in the next paragraph, such a lemma is false if $X$ is strictly DMsemistable and the subcurve with extremal degree is a destabilizing component. 
Lemma 3.1. Let $X \subset \mathbb{P}^{r}$ be a DM-semistable curve; assume that there is a complete subcurve $Y$ of $X$ such that

$$
d_{Y}=\frac{d}{(g-1)}\left(g_{Y}-1+k_{Y} / 2\right)-k_{Y} / 2
$$

and such that $\overline{X \backslash Y}$ is not a union of destabilizing components. Then $\operatorname{hilb}(X)$ is not G-stable.

Proof. We will first assume that $X$ has only two smooth irreducible components, $Y$ and $Z$, meeting at $k$ points, and such that

$$
d_{Y}=m_{Y} \text { and } d_{Z}=M_{Z} \text {. }
$$

It is convenient to recall that from the first part of the proof of Proposition 3.1 we can derive

$$
\text { (*) } \quad d_{Y}=\frac{d}{(g-1)}\left(g_{Y}-1+k_{Y} / 2\right)-k_{Y} / 2 \Longleftrightarrow \frac{h^{0}\left(Y, L_{Y}\right)}{h^{0}(X, L)}=\frac{d_{Y}+k / 2}{d} .
$$

We will use the same $\lambda$ and the same notation as in the proof of the previous proposition. By the numerical criterion of stability (see 1.4), to prove that $h$ is not stable it is enough to prove that there exists a $\lambda$ such that

$$
T=\sum_{j=1}^{p(m)} w_{\lambda}\left(M_{j}\right) \geq \frac{\sum_{0}^{r} w_{j}}{h^{0}(X, L)} m p(m)
$$

for every $p(m)$-uple of monomials which restrict to a basis of $H^{0}\left(X, L^{m}\right)$.

Remark. We will see in the sequel that $h$ is in fact $G$-semistable, that is, the inequality above will be an equality.

We have already obtained the estimate

$$
T \geq m b_{m}-\sum_{i=0}^{m-1}\left(m d_{Z}+(i-m) k-g_{Z}+1+h_{i}^{1}\right),
$$

but now

$$
h_{i}^{1}=h^{1}\left(Z, L_{Z}^{m}(i-m) D\right)=0
$$

because

$$
\operatorname{deg} L_{Z}^{m}(i-m) D=m\left(d_{Z}-k\right)+i k>2 g_{Z}-2 ;
$$

in fact, since we are assuming that $g_{Z}=0$ implies $k \geq 3$, Proposition 3.1 yields that $d_{z}>k$. Hence, we have

$$
T \geq m^{2}\left(d_{Y}+k / 2\right)+m\left(1-g+k / 2+g_{Z}-1\right) ;
$$

on the other hand, by $(*)$

$$
m p(m) \frac{\sum w_{j}}{d-g+1} \leq m p(m) \frac{h^{0}\left(Y, L_{Y}\right)}{d-g+1}=m^{2}\left(d_{Y}+k / 2\right)+m\left(-g_{Y}+1-k / 2\right) .
$$


Finally, we can conclude

$$
m p(m) \frac{\sum w_{j}}{d-g+1} \leq T .
$$

This proves the lemma in the special case of a curve with only two smooth irreducible components; let us now consider the general case.

Let $Y \subset X$ and let $Z$ be the complementary curve, so that

$$
d_{Y}=m_{Y} \text { and } d_{Z}=M_{Z} \text {. }
$$

We start by observing that one can assume that both $Y$ and $Z$ are connected, because all connected components of $Y$ (respectively $Z$ ) must have minimum (resp. maximum) degree. In fact, let

$$
Y=\cup Y_{i}, \quad Y_{i} \cap Y_{j}=\varnothing \quad \forall i \neq j
$$

then

and analogously for $Z$. Now, let

$$
m_{Y}=\sum m_{Y_{i}} \text { hence } d_{Y_{i}}=m_{Y_{i}}
$$

$$
W=\overline{X \backslash Y_{1}}=\cup W_{i},
$$

$W_{i}$ connected components; then

$$
d_{W_{i}}=M_{W_{i}} \forall i
$$

so that we can take

$$
Y^{\prime}=Y_{1} \cup\left(\cup_{i \geq 2} W_{i}\right) \text { and } Z^{\prime}=W_{1}
$$

provided that $W_{1}$ is not a destabilizing component, which we can certainly assume.

Now $Y^{\prime}$ and $Z^{\prime}$ are connected and they have extremal degrees.

Let $L:=\mathscr{O}_{\mathbb{P}^{\prime}}(1) \otimes \mathscr{O}_{X}$, and consider a generic one-parameter deformation of $(X, L)$ which is locally trivial at $Y \cap Z$. The generic fiber of it is a DM-stable curve having two smooth irreducible components meeting at $|Y \cap Z|$ points. We have already proved that the Hilbert point of it is not stable.

Since the locus of nonstable points in Hilb ${ }_{r}^{p(x)}$ is closed, we can conclude that the Hilbert point of the special fiber is also nonstable.

3.3. Quasistable curves and ladders. We will now apply Proposition 3.1 to give a more detailed description of the points of $H_{d}$.

If $h=\operatorname{hilb}(X, L) \in H_{d}$, by applying Proposition 3.1 to any chain of destabilizing components of $X$, we get that the degree of the restriction of $L$ to such a subcurve is 1 . In other words, every destabilizing chain of $X$ is actually an irreducible rational curve which is mapped by $L$ to a line of $\mathbb{P}^{r}$. This observation motivates the following definition:

Definition. We will call a DM-semistable curve quasistable if all its destabilizing chains have length 1 . 
We have just seen that all points of $H_{d}$ correspond to quasistable curves. It is important to observe that, although a strictly quasistable curve $X$ has a positive dimensional automorphism group as an abstract curve, it might very well happen that its models of degree $d$ in $\mathbb{P}^{r}$ have only finitely many linear automorphisms. More precisely, recall that $D_{X}$ denotes the subcurve of $X$ given by the union of all destabilizing components of $X$, and let $\operatorname{Stab}_{G}(h)$ be the stabilizer of $h$ in $G=S L(r+1)$. Then we can prove the following

Lemma 3.2. Let $h=\operatorname{hilb}(X) \in H_{d}$; then $\operatorname{Stab}_{G}(h)$ has positive dimension if and only if $\overline{X \backslash D_{X}}$ is not connected.

Proof. Since $h \in H_{d}, X$ is not contained in any hyperplane of $\mathbb{P}^{r}$ (by Gieseker's Theorem). Let $Y=\overline{X \backslash D_{X}}$; since $D_{X}$ is a union of lines, $Y$ and $X$ span the same linear subspace of $\mathbb{P}^{r}$, that is, $Y$ spans $\mathbb{P}^{r}$.

Suppose that $Y$ is connected; since $Y$ is also nondegenerate, every $\alpha \in$ $\operatorname{Stab}_{G}(h)$ is determined (up to a finite subgroup of $G$ ) by its restriction to $Y$. Now, either $g_{Y}>1$, hence $Y$ is DM-stable and $\operatorname{Aut}(Y)$ is finite; or $g_{Y} \leq 1$ in which case $Y$ has

$$
\rho=\frac{\left|D_{X} \cap Y\right|}{2}
$$

marked couples of points which are fixed by $\alpha$. If $g_{Y}=1$ then $\rho>1$, and if $g_{Y}=0$ then $\rho \geq 3$; in both cases there are only finitely many automorphisms of $Y$ which fix that many couples. Hence $\operatorname{Stab}_{G}(h)$ is finite.

Viceversa, suppose $Y=Y_{1} \cup Y_{2}, Y_{1} \cap Y_{2}=\varnothing$, where $Y_{i}$ are complete subcurves of $Y$. Let $\Lambda_{i}$ be the linear subspace spanned by $Y_{i}$. It is $\Lambda_{1} \cap \Lambda_{2}=\varnothing$, since, by the Riemann-Roch Theorem, we have $\operatorname{dim} \Lambda_{1}+\operatorname{dim} \Lambda_{2}=r-1$. Therefore, there exists a positive dimensional family of automorphisms of $\mathbb{P}^{r}$ which fix both $\Lambda_{i}$; the nontrivial elements of such a family will induce nontrivial automorphisms on $D_{X}$; therefore $\operatorname{Stab}_{G}(h)$ has positive dimension.

Definition. A quasistable curve $X$ which satisfies the condition in the above lemma (that is, $\overline{X \backslash D_{X}}$ is not connected) will be called a ladder. The number

$$
\#\left\{\text { connected components of } \overline{X \backslash D_{X}}\right\}-1
$$

will be called the order of $X$. A ladder could also be defined as a curve having positive order.

This somewhat funny name "ladder" is suggested by the picture of the case in which $\overline{X \backslash D_{X}}$ has two irreducible nonsingular components, $C_{1}$ and $C_{2}$ (see Figure 1 on the next page).

If $h \in \mathrm{Hilb}_{r}^{p(x)}$, let $O_{G}(h)$ be the orbit of $h$ under the $G$-action. Since $\operatorname{dim} O_{G}(h)=\operatorname{dim} G-\operatorname{dim} \operatorname{Stab}_{G}(h)$, we have that $\operatorname{dim} O_{G}(h)<r(r+2)$ if and only if $X_{h}$ is a ladder.

3.3.1. Important remark. Notice that if $X \subset \mathbb{P}^{r}$ is a ladder having semistable Hilbert point, every connected component $Y$ of $\overline{X \backslash D_{X}}$ satisfies

$$
d_{Y}=m_{Y}
$$




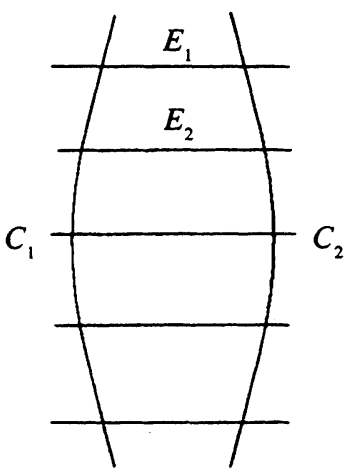

FIGURE 1

\section{COMBINATORIAL CONSIDERATIONS}

The purpose of this section is to introduce a language which will be used to deal with the combinatorial problem.

4.1. The degree class group of a nodal curve. Let $X=\cup_{i=1}^{\gamma} C_{i}$ be a DM-semistable curve and let the $C_{i}$ be its irreducible components; let $d$ be an integer. In this section, we will assume the above data to be fixed.

If $L$ is a line bundle of degree $d$ on $X$ we define its multidegree $\underline{d}$ to be the ordered $\gamma$-uple of integers

$$
\underline{\operatorname{deg}} L=\underline{d}=\left(d_{1}, \ldots, d_{\gamma}\right) \text { where } d_{i}=\operatorname{deg}_{C_{i}} L \text { and } \sum_{i=1}^{\gamma} d_{i}=d .
$$

We will denote by $\operatorname{Pic}_{X}^{\frac{d}{X}}$ the variety of line bundles of multidegree $\underline{d}$ on $X$. Given $L \in \operatorname{Pic}_{X}^{\frac{d}{}}$, Proposition 3.1 imposes a necessary condition for $\operatorname{hilb}(X, L)$ to be semistable. It is convenient to introduce the next

Definition. Let $L \in \operatorname{Pic}_{X}^{\frac{d}{X}}$ be a line bundle on a DM-semistable curve. We say that $L$ and $\underline{d}$ satisfy the Basic Inequality if and only if every subcurve of $X$ satisfies (BI) in Theorem 3.1, with respect to $L$. We will denote by $B_{X}^{d}$ (or, more often, simply $B_{X}$ ) the set of multidegrees $\underline{d}$ satisfying the Basic Inequality on $X$ and such that $d_{i} \geq 0 \forall i$.

In this section, we will illustrate in what sense the Basic Inequality is actually sharp. We will start by associating to every curve a finite group which is an invariant of its combinatorial data. There is a natural equivalence relation among all the multidegrees summing to a fixed $d$. This comes from the fact that in any deformation of $X$ with smooth total space $\mathscr{X}$ over a smooth onedimensional base, one can twist a line bundle $\mathscr{L}$ on the total space by another line bundle of type $\mathscr{O}_{\mathscr{Z}}\left(\sum_{i=1}^{\gamma} r_{i} C_{i}\right), r_{i} \in \mathbb{Z}$, having only the effect of changing the restriction of $\mathscr{L}$ to $X$ and its multidegree. The point is that the way the multidegree of $\mathscr{L}_{\mid X}$ changes does not depend on the choice of the polarized family $(\mathscr{X}, \mathscr{L})$; it only depends on the ordered $\gamma$-uple $\left(r_{1}, \ldots, r_{\gamma}\right)$. 
To precisely define this equivalence relation, we only need to consider the combinatorial data of $X$; define

$$
k_{i, j}= \begin{cases}\left|C_{i} \cap C_{j}\right|, & \text { if } i \neq j, \\ \left|C_{i} \cap \overline{X \backslash C_{i}}\right|, & \text { if } i=j\end{cases}
$$

and let $\mathbf{Z} \cong \mathbb{Z}^{\gamma-1}$ be

$$
\mathbf{Z}=\left\{\left(r_{1}, \ldots, r_{\gamma}\right\} \in \mathbb{Z}^{\gamma}, \text { such that } \sum_{i=1}^{\gamma} r_{i}=0\right\} ;
$$

let $\Lambda_{X} \subset \mathrm{Z}$ be the lattice generated by the $\gamma$ vectors

$$
\begin{aligned}
& \underline{c}_{1}=\left(-k_{1,1}, k_{1,2}, \ldots, k_{1, \gamma}\right) \\
& \underline{c}_{i}=\left(k_{i, 1}, \ldots,-k_{i, i}, \ldots, k_{i, \gamma}\right) \\
& \underline{c}_{\gamma}=\left(k_{\gamma, 1}, k_{\gamma, 2}, \ldots,-k_{\gamma, \gamma}\right) ;
\end{aligned}
$$

of course, these vectors are not independent, since

$$
\sum_{j=1}^{\gamma} \underline{c}_{j}=0 .
$$

The meaning of these vectors is clear: $\underline{c}_{i}$ corresponds to the multidegree of the restriction to $X$ of the line bundle $\mathscr{O}_{\mathscr{L}}\left(C_{i}\right)$, where $\mathscr{Z}$ is the total space of a generic one-parameter deformation of $X$. We will use the same suggestive notation for any complete subcurve $Y$ of $X$, that is, we will denote by $\underline{y}$ the multidegree of any line bundle of type $\mathscr{O}_{\mathscr{D}}(Y)$ on $X$.

Now, if we are given $\underline{d}=\left(d_{1}, \ldots, d_{\gamma}\right)$ and $\underline{d}^{\prime}=\left(d_{1}^{\prime}, \ldots, d_{\gamma}^{\prime}\right)$ with $\sum d_{i}=$ $\sum d_{i}^{\prime}=d$, we say that $\underline{d}$ is equivalent to $\underline{d}^{\prime}$ (with respect to $X$ ) if $\underline{d}-\underline{d}^{\prime}$ lies in $\Lambda_{X}$, where $\underline{d}-\underline{d}^{\prime}=\left(d_{1}-d_{1}^{\prime}, \ldots, d_{\gamma}-d_{\gamma}^{\prime}\right)$. The notation will be $\underline{d} \equiv \underline{d}^{\prime}$.

Definition. The degree class group of $X$ is the finite group $Z / \Lambda_{X}$, which will be denoted by $\Delta_{X}$.

Remark. The group $\mathbf{Z} / \Lambda_{X}$ is equal to the space $\Delta_{X}^{0}$ of equivalence classes of multidegrees which sum to zero. This is, of course, noncanonically isomorphic to the space $\Delta_{X}^{d}$ of equivalence classes of multidegrees summing to $d$, for any integer $d$. In order to simplify the notation, we will still denote by $\Delta_{X}$ such a space, without further specifying $d$. We can just assume that we fixed an isomorphism between $\Delta_{X}$ and $\Delta_{X}^{d}$, for every $d$, once and for all.

The cardinality of such a group will be denoted by $\mu(X)$. We will show later (Corollary 5.1) that $\mu(X)$ is an upper bound for the number of irreducible components of $\overline{P_{d, X}}$. Moreover, if $X$ has trivial automorphism group, there will be values of $d$ for which $\overline{P_{d, X}}$ has exactly $\mu(X)$ irreducible components. 
If $X$ has automorphisms which permute its components, then a sharp upper bound would be given by the cardinality of the quotient of $\Delta_{X}$ by the automorphism group of $X$.

Examples. (1) If $X$ has only two irreducible components $C_{1}$ and $C_{2}$, with $\left|C_{1} \cap C_{2}\right|=k$, then $\underline{c}_{1}=(-k, k), \quad \underline{c}_{2}=(k,-k)$, and

$$
\Delta_{X} \cong \mathbb{Z} / k \mathbb{Z} \text {. }
$$

(2) If $X$ has $\gamma$ irreducible components $C_{1}, \ldots, C_{\gamma}$, then the cardinality of $\Delta_{X}$ will be given by the absolute value of the determinant of the $(\gamma-1) \times(\gamma-1)$ intersection matrix relative to any choice of $\gamma-1$ components of $X$. In other words,

$$
\left|\Delta_{X}\right|=\left|\operatorname{det}\left(\left(C_{i} \cdot C_{j}\right)\right)_{\substack{i=1, \ldots, y-1 \\ j=1, \ldots, y-1}}\right| \cdot
$$

Let us apply this to a quasistable curve obtained by destabilizing all the $\delta$ nodes of an irreducible curve, that is, $X$ has $\gamma=\delta+1$ components, $\delta$ of which are destabilizing and do not intersect each other. We obtain that the degree class group of $X$ has $2^{\delta}$ elements.

4.2. Relation with Geometric Invariant Theory. Recall that if $Y$ is a subcurve of $X$, we denoted the extremes of the Basic Inequality by

$m_{Y}=\frac{d}{(g-1)}\left(g_{Y}-1+k_{Y} / 2\right)-k_{Y} / 2, \quad M_{Y}=\frac{d}{(g-1)}\left(g_{Y}-1+k_{Y} / 2\right)+k_{Y} / 2$

where $g_{Y}$ is the arithmetic genus of $Y$.

We want to start by a simple observation. First if $A$ and $B$ are two subcurves of $X$ with no common components, we define

$$
k_{A, B}:=|A \cap B| \text {. }
$$

Then if $W=A \cup B$, the Basic Inequality yields

$$
m_{W}=m_{A}+m_{B}+k_{A, B} \text { and } M_{W}=M_{A}+M_{B}-k_{A, B} .
$$

We have the following

4.2.1. Important remark. If $\underline{d}$ satisfies the Basic Inequality and $Z$ is a subcurve of $X$ such that $d_{Z}=M_{Z}$, then $\underline{d}+\underline{z}$ also satisfies the Basic Inequality.

To check this, let $\underline{d}^{\prime}=\underline{d}+\underline{z}$ and let $Y=\overline{X \backslash Z}$. Assume that there exists a subcurve $W$ of $X$ such that $d_{W}^{\prime}>M_{W}$; let us write $W=A \cup B$ with $A \subset Y$ and $B \subset Z$. We have

$$
d_{W}^{\prime}=d_{A}+k_{A, Z}+d_{B}-k_{B, Y}>M_{W}=M_{A}+M_{B}-k_{A, B} .
$$

Since $\underline{d}$ satisfies the Basic Inequality, we obtain

$$
d_{A}>M_{A}-k_{A, B}-k_{A, Z}+k_{B, Y}>M_{A}-k_{A, Z} \text {. }
$$

This gives

which is a contradiction.

$$
d_{A \cup Z}>M_{A}+M_{Z}-k_{A, Z}=M_{A \cup Z},
$$


It is straightforward to check that, in the case of a curve having only two irreducible components, every degree class has a representative which satisfies the Basic Inequality. In fact, according to Theorem 3.1, if the two components of the curve meet in $k$ points, then the length of the interval in which the degree of either one component can vary has length $k+1$. On the other hand, the cardinality of the degree class group is $k$, and two multidegrees $\underline{d}=\left(d_{1}, d_{2}\right)$ and $\underline{d}^{\prime}=\left(d_{1}^{\prime}, d_{2}^{\prime}\right)$ are equivalent if and only $d_{1}=d_{1}^{\prime}+n k$ for some integer $n$. Hence, for any fixed degree class, at least one representative (and at most two) will fall in the allowable range.

The same is true for the general quasistable curve, but the proof, although elementary, is more complicated:

Proposition 4.1. Let $X$ be a quasistable curve and $\Delta_{X}$ its degree class group. Then every element $\delta$ of $\Delta_{X}$ has a representative which satisfies the Basic Inequality. Proof. Given any representative $\underline{d}$ for $\delta$, we will exhibit a method to produce a new multidegree which satisfies the Basic Inequality and which is also a representative for $\delta$. Suppose $\underline{d}$ does not satisfy the Basic Inequality; then there exists a complete proper subcurve $Y$ of $X$ for which the condition of Theorem 3.1

$$
m_{Y} \leq d_{Y} \leq M_{Y}
$$

is violated, that is (for example), $d_{Y}>M_{Y}$; we will call the number $d_{Y}-M_{Y}$ the "excess" of $Y$ with respect to $\underline{d}$ and we will denote it by $\epsilon_{Y}(\underline{d})$ or just $\epsilon_{Y}$. We have (by the observation at the beginning of the section)

$$
\epsilon_{W}=\epsilon_{A}+\epsilon_{B}+k_{A, B}
$$

this implies that there exists a unique curve $Z(\underline{d})=Z$ having maximum excess and minimal with respect to this property (that is, the excess of any proper subcurve of $Z$ is less than the excess of $Z$ ). In fact, if $Z_{1}$ and $Z_{2}$ are two different curves with the above property, then the union of them,

$$
U=Z_{1} \cup Z_{2},
$$

will have excess

$$
\epsilon_{U}>\epsilon_{Z_{1}}
$$

contradicting the assumption that $Z_{1}$ has maximum excess. This is clear if $Z_{1}$ and $Z_{2}$ have no common components. If, instead, $L$ is the curve in which they intersect, let $H=\overline{Z_{2} \backslash L}$; then we have

$$
\epsilon_{U}=\epsilon_{Z_{1}}+\epsilon_{H}+k_{Z_{1}, H}
$$

But, by the minimality of $Z_{2}$

$$
\epsilon_{H}+k_{H, L}>0 \text { hence a fortiori } \epsilon_{U}>\epsilon_{Z_{1}} .
$$

We will define

$$
\epsilon(\underline{d})=\epsilon_{Z(\underline{d})}
$$

to be the total excess of $\underline{d}$, and we will call $Z$ the excess curve of $\underline{d}$. We will 
make the convention that the excess of the empty curve be equal to 0 ; in this way,

$$
Z(\underline{d})=\varnothing \Longleftrightarrow \underline{d} \text { satisfies the Basic Inequality } \Longleftrightarrow \epsilon(\underline{d})=0 .
$$

The proof of the proposition will consist of showing a way by which, given a $\underline{d}$ which does not satisfy the Basic Inequality, we can find a new multidegree $\underline{d}^{\prime} \equiv \underline{d}$ which has smaller total excess.

Note that for any complete subcurve $Y$ of $X$, we have

$$
Y \subset Z \Longrightarrow d_{Y}-M_{Y}+k_{Y, Z}>0 \text {. }
$$

In the same way we defined $Z(\underline{d})$, we can define the deficiency curve $A=A(\underline{d})$ as the minimal subcurve of $X$ having maximum deficiency, where the deficiency of a subcurve $Y$ will be defined as the number $m_{Y}-d_{Y}$. It is easy to see that $A$ and $Z$ have no common components.

Finally, we have a decomposition

$$
X=Z(\underline{d}) \cup N(\underline{d}) \cup A(\underline{d})=Z \cup N \cup A
$$

where $N$ is a sort of "neutral" subcurve, defined to be

$$
N:=\overline{X \backslash(A \cup Z)} \text {. }
$$

We have that

$$
Y \subset A \Longrightarrow-d_{Y}+m_{Y}+k_{Y, A}>0 \Longleftrightarrow d_{Y}-M_{Y}+k_{Y, Z}<-k_{Y, N} .
$$

Now, we claim that, if $A \cap Z \neq \varnothing$, then the operation of twisting by $Z$ actually yields a multidegree $\underline{d}^{\prime}$ having smaller total excess. In other words, let

$$
\underline{d}^{\prime}=\underline{d}+\underline{z}
$$

then, if $A \cap Z \neq \varnothing$, we will prove that

$$
\epsilon\left(\underline{d}^{\prime}\right)<\epsilon(\underline{d}) \text {. }
$$

If $Y$ is a subcurve of $X$, we will write

$$
\epsilon_{Y}\left(\underline{d}^{\prime}\right)=\epsilon_{Y}^{\prime}
$$

we have to show that, for any $Y, \epsilon_{Y}^{\prime}<\epsilon(\underline{d})$. We can decompose $Y$ as

$$
Y=W \cup L \cup B \text { with } \varnothing \subseteq W \subseteq Z, \varnothing \subseteq L \subseteq N, \varnothing \subseteq B \subseteq A .
$$

Then we have

$$
\epsilon_{Y}^{\prime}=\epsilon_{W}^{\prime}+\epsilon_{L}^{\prime}+\epsilon_{B}^{\prime}+k_{W, L}+k_{L, B}+k_{B, W}
$$

Now,

$$
\epsilon_{W}^{\prime}=d_{W}-k_{W, N}-k_{W, A}-M_{W} \leq \epsilon_{Z}-k_{W, N}-k_{W, A}
$$

where equality holds if and only if $Z=W$, because of the minimality of $Z$,

$$
\epsilon_{L}^{\prime}=d_{L}+k_{L, Z}-M_{L} \leq 0
$$


and finally

$$
\epsilon_{B}^{\prime}=d_{B}+k_{B, Z}-M_{B} \leq-k_{B, M}
$$

where equality holds if and only if $B$ is empty. Therefore

$$
\epsilon_{Y}^{\prime} \leq \epsilon_{Z}-k_{W, N}-k_{W, A}-k_{B, N}+k_{W, B}+k_{W, L}+k_{B, L} \leq \epsilon_{Z}
$$

where equality holds if and only if $W=Z$ and $B=\varnothing$. Therefore we are left with $Y=Z \cup L$, where

$$
\epsilon_{Y}^{\prime} \leq \epsilon_{Z}-k_{Z, N}-k_{Z, A}+k_{Z, L}
$$

and clearly the only case in which $\epsilon_{Y}^{\prime}=\epsilon_{Z}$ is when $Y=Z \cup N$ and $k_{A, Z}=0$, i.e., $A \cap Z=\varnothing$.

Our claim is then proved; to conclude we need to deal with the case $A \cap Z=$ $\varnothing$, when we saw that $\epsilon\left(\underline{d}^{\prime}\right)=\epsilon(\underline{d})=\epsilon_{Z \cup M}^{\prime}$.

Let us consider

$$
\underline{d}^{\prime \prime}=\underline{d}^{\prime}+\underline{n}+\underline{z}
$$

we will see that the total excess of $\underline{d}^{\prime \prime}$ is smaller than that of $\underline{d}$. As before, with analogous notation, we have

$$
\begin{aligned}
\epsilon_{Y}^{\prime \prime} & =\epsilon_{W}^{\prime \prime}+\epsilon_{L}^{\prime \prime}+\epsilon_{B}^{\prime \prime}+k_{W, L}+k_{L, B}+k_{B, W} \\
& =\left(\epsilon_{W}^{\prime}\right)+\left(\epsilon_{L}^{\prime}-k_{L, A}\right)+\left(\epsilon_{B}^{\prime}+k_{B, N}\right)+k_{B, L}+k_{W, L}
\end{aligned}
$$

finally, since $\epsilon_{L}^{\prime} \leq 0$ and $\epsilon_{B}^{\prime}=\epsilon_{B} \leq-k_{B, N}$ (equality holds iff $B$ is empty), we have

$$
\epsilon_{Y}^{\prime \prime} \leq \epsilon_{W}-k_{W, N}-k_{L, A}+k_{W, L}+k_{B, L}<\epsilon_{Z}
$$

hence $\epsilon\left(\underline{d}^{\prime \prime}\right)<\epsilon(\underline{d})$.

Notice that, by 4.2.1, a multidegree class has a representative satisfying the Basic Inequality if and only if it has a representative in $B_{X}^{d}$ (cf. Definition in Section 4.1). In fact, if $\underline{d}$ satisfies the Basic Inequality, but it is negative on a subcurve $C$ of $X$, then $C$ must be a union of destabilizing components and hence, by $4.2 .1, \underline{d}+\underline{c}$ will be in $B_{X}^{d}$.

4.3. A useful normalization. Let $\underline{v}=\left(v_{1}, \ldots, v_{\gamma}\right)$ be an element of $\Lambda_{X}$; then $\underline{v}$ can be written as a combination of the $\underline{c}_{i}$ in many different ways. Yet there is a convenient choice that can be made, if $\underline{v}$ is such that $\left|v_{i}\right| \leq k_{i, i}$ for every $i$. This is explained in the following simple lemma.

Lemma 4.1. Let $X=\cup_{1}^{\gamma} C_{i}$ be a DM-semistable curve. Let $\underline{d}$, $\underline{d}^{\prime}$ be two multidegrees on $X$ which satisfy the Basic Inequality, and such that $\underline{d} \equiv \underline{d}^{\prime}$. Then 
there exists a partition in disjoint subsets

$$
\{1,2, \ldots, \gamma\}=J_{0} \cup J_{1} \cup \cdots \cup J_{q},
$$

such that

$$
X_{J_{h}} \cap X_{J_{k}}=\varnothing \quad \Longleftrightarrow|h-k| \geq 2
$$

and

$$
\underline{d}-\underline{d}^{\prime}=\sum_{j \in J_{1}} \underline{c}_{j}+\sum_{j \in J_{2}} 2 \underline{c}_{j}+\cdots+\sum_{j \in J_{q}} q \underline{c}_{j}
$$

where $q$ is some positive integer.

Proof. Even without assuming that $\underline{d}$ and $\underline{d}^{\prime}$ satisfy the Basic Inequality, we can write

$$
\underline{d}-\underline{d}^{\prime}=\sum_{h=0}^{q} \sum_{j \in J_{h}} h \underline{c}_{j} .
$$

In fact, for any element $\underline{v}$ of $\Lambda_{X}$ we have

$$
\underline{v}=\sum_{i=1}^{\gamma} u_{i} \underline{c}_{i}, \quad u_{i} \in \mathbb{Z}
$$

Let $m=\min \left\{u_{i}\right\}$ and let us define

$$
J_{0}=\left\{i: 1 \leq i \leq \gamma, \quad u_{i}=m\right\} ;
$$

clearly $J_{0}$ is not empty. Now we have the relation

$$
\sum_{j \in J_{0}} \underline{c}_{j}=-\sum_{i \notin J_{0}} \underline{c}_{i}
$$

hence

$$
\underline{v}=\sum_{i=1}^{\gamma}\left(u_{i}-m\right) \underline{c}_{i}=\sum_{i=1}^{\gamma} v_{i} \underline{c}_{i}
$$

and clearly now $v_{i} \geq 0$. Let

$$
J_{h}=\left\{i: 1 \leq i \leq \gamma, \quad v_{i}=h\right\} .
$$

Now, if $\underline{d}$ and $\underline{d}^{\prime}$ satisfy the Basic Inequality, we will prove that

$$
X_{J_{h}} \cap X_{J_{k}}=\varnothing \Longleftrightarrow|h-k| \geq 2 .
$$

Let $I=J_{0}$ and $J=J_{1} \cup \cdots \cup J_{M}$; then by Theorem 3.1

$$
k_{I, J} \geq\left|d_{I}-d_{I}^{\prime}\right|=\left|\sum_{j \in J} v_{j} k_{I, j}\right| .
$$

Since $k_{I, J}=\sum_{j} k_{I, j}$ we find that if $k_{I, j} \neq 0$ then $v_{j}=1$; that is, $X_{J_{0}} \cap X_{J_{h}}=$ $\varnothing \forall h \geq 2$. 
Now we can conclude by induction: let $l: 2 \leq l \leq q$, and let $I=J_{0} \cup \cdots \cup J_{l}$ and $J=J_{l+1} \cup \cdots \cup J_{q}$. Arguing exacly like before, we have that

$$
k_{I, J} \geq\left|d_{I}-d_{J}^{\prime}\right|=\left|-l k_{I, J}+\sum_{j \in J} v_{j} k_{I, j}\right| \geq\left|-l k_{I, J}+(l+1) \sum_{j \in J} k_{I, j}\right|=k_{I, J}
$$

Therefore, since equality holds if and only if $v_{j}=l+1 \forall j: k_{I, j} \neq 0$, the proof of the lemma is finished.

This lemma gives us a recipe to reduce the case of a general curve to the combinatorially simple case of a curve having only two irreducible components. In fact, given $\underline{d}$ and $\underline{d}^{\prime}$ in the same multidegree class and both satisfying the Basic Inequality, the lemma gives us a decomposition of $X$

$$
X=\cup_{h=0}^{q} X_{J_{h}}
$$

such that for any $l, 0 \leq l \leq q-1$, we can define two (complementary) subcurves of $X$

$$
A_{l}=\cup_{h=0}^{l} X_{J_{h}} \text { and } B_{l}=\cup_{h=l+1}^{M} X_{J_{h}}
$$

with $A_{i} \cap B_{j}=\varnothing$ if $j>i$. We have

$$
d_{A_{l}}-d_{A_{l}}^{\prime}=k_{l}
$$

where $k_{l}=\left|A_{l} \cap B_{l}\right|=\left|X_{J_{l}} \cap X_{J_{l+1}}\right|$. This is saying that we can obtain $\underline{d}^{\prime}$ from $\underline{d}$ by twisting by one (reduced) subcurve at the time, in such a way that these "twists"do not interfere with each other. We can write, in a fashion that we will call the "normal form" of $\underline{v}$,

$$
\underline{v}=\underline{d}-\underline{d}^{\prime}=\sum_{l=0}^{q-1} \underline{b}_{l}
$$

or

$$
\underline{v}=\sum_{h=1}^{q} h \cdot \underline{x}_{J_{h}}
$$

Notice finally that we have $d_{B_{l}}=m_{B_{l}}$ and $d_{A_{l}}=M_{A_{l}}$.

The following corollary will be used in the sequel. The proof is an immediate consequence of the previous lemma.

Corollary 4.1. Let $X=\cup_{1}^{\gamma} C_{i}$ be a DM-semistable curve. Let $\underline{d}$, $\underline{d}^{\prime}$ be two multidegrees on $X$ which satisfy the Basic Inequality, and such that $\underline{d} \equiv \underline{d}^{\prime}$. Let $E$ be a destabilizing component of $X$ such that $d_{E}=0$ and $d_{E}^{\prime}=1$. Then there exists $h \geq 0$ such that

$$
E \subset X_{J_{h}}, \quad\left|E \cup X_{J_{h-1}}\right|=1, \quad E \cap X_{J_{h+1}}=\varnothing .
$$

4.4. Locally trivial deformations. The degree class group of $X$ does not depend on the choice of a deformation, and we introduced it as a tool to measure the 
ambiguity of the limiting line bundle. Such an ambiguity will in fact be faithfully reflected in $\Delta_{X}$, provided that we limit ourselves to general deformations, which in particular will not be locally trivial at the nodes of $X$. In the sequel, we will consider deformations of a given curve which preserve some of its nodes; such families will still be nice, in the following sense:

Definition. Let $\mathscr{X} \longrightarrow B$ be a one-parameter deformation of the DM-semistable curve $X$. We say that such a family is a generic locally trivial deformation of $X$ if $B$ is smooth, $\mathscr{Z}$ is reduced, it preserves some subset of the nodes of $X$, and has no other singularities than the ones corresponding to such nodes. Moreover we require that such singular curves be simple double curves, that is, they are locally given by transverse intersection of two branches of $\mathscr{Z}$.

The simplest example of such a deformation is, of course, a trivial family $X \times B \rightarrow B$.

Let $\mathscr{Z} \longrightarrow B$ be a one-parameter deformation of $X$. We say that a node $N$ of $X$ is generic if $\mathscr{Z}$ is locally trivial at $N$. We say that $N$ is special if $\mathscr{Z}$ is smooth at $N$.

As we said, we will always reduce ourselves to deformations $\mathscr{Z}$ of $X$ such that a node $N$ of $X$ is either a smooth point of $\mathscr{Z}$, or $\mathscr{Z}$ is locally trivial at $N$. That is to say that a node of $X$ is either special or generic.

Then we have the following observation:

4.4.1. Important remark. Let $\mathscr{X} \longrightarrow B$ be a one-parameter deformation of $X$ as above; then not every subcurve $Y$ of $X$ will determine a line bundle on $\mathscr{Z}$. More precisely, the sheaf $\mathscr{O}_{\mathscr{L}}(Y)$ is locally free if and only if all nodes of $Y \cap \overline{X \backslash Y}$ are special.

\section{THE FIBERS OF $\psi_{d}$}

5.1. Deforming quasistable curves in $\mathbb{P}^{r}$. Recall that we already defined, for any DM-semistable curve $X$, the set of its GIT-semistable projective models, denoted by $M_{X} \cdot M_{X}$ can be decomposed in a natural way as

$$
M_{X}=\cup_{\underline{d}} M_{X}^{\underline{d}}
$$

where

$$
M_{x}^{\underline{d}}=\left\{h \in H_{d}, h=\operatorname{hilb}(X, L) \text { s.t. } \underline{\operatorname{deg}} L=\underline{d}\right\} .
$$

Of course, $M_{X}^{\underline{d}}$ will be empty for all but finitely many $\underline{d}$, since the Basic Inequality only allows a finite number of multidegrees on a $G$-semistable model of $X$. Let us now define

$$
V_{x}^{\underline{d}}=\overline{M_{x}^{\underline{d}}} \cap H_{d}
$$

(in other words, $V_{x}^{\underline{d}}$ is the semistable closure of $M_{x}^{\underline{d}}$ ) and let

$$
V_{X}=\cup_{\underline{d}} V_{x}^{\underline{d}}
$$

be the semistable closure of $M_{X}$. 
If $X$ is DM-stable, $V_{X}$ is clearly contained in the fiber over $[X]$ of the morphism

$$
\psi_{d}: H_{d} \longrightarrow \bar{M}_{g} .
$$

The expectation is that $V_{X}$ is in fact equal to $\psi_{d}^{-1}([X])$. This amounts to saying that $\psi_{d}^{-1}([X])$ does not have any extra irreducible component, other than the $V_{x}^{\underline{d}}$. In the subsequent proposition we will show that this is exactly what happens, by constructing a degeneration of $X$ to $X_{h^{\prime}}$ for any $h^{\prime} \in \psi^{-1}([X])$. In fact the proof will give us also all the multidegrees on $X$ such that $h^{\prime}$ is contained in the corresponding irreducible component of $V_{X}$.

Proposition 5.1. Let $X=\cup_{1}^{\gamma} C_{i}$ be a quasistable curve and let the $C_{i}$ be its irreducible components. Let $h^{\prime} \in H_{d}, h^{\prime}=\operatorname{hilb}\left(X^{\prime}, L^{\prime}\right)$, with $L^{\prime} \in \mathrm{Pic}_{X^{\prime}}$. Then $h^{\prime} \in V_{x}^{\underline{d}}$ if and only if the following condition holds:

$X^{\prime}$ is obtained by destabilizing $\rho$ nodes of $X$, in such a way that we can write

$$
X^{\prime}=\left(\cup_{1}^{\gamma} C_{i}^{\prime}\right) \cup\left(\cup_{1}^{\rho} E_{j}\right)
$$

where the $E_{j}$ are the new destabilizing components and $C_{i}^{\prime}$ has a birational morphism to $C_{i}$; moreover, there is a decomposition of $X^{\prime}$

$$
X^{\prime}=\cup_{1}^{\gamma} C_{i}^{\prime \prime}
$$

such that $C_{i}^{\prime \prime}$ is a complete connected subcurve of $X^{\prime}$ and

(1) $C_{i}^{\prime} \subseteq C_{i}^{\prime \prime} \quad \forall i$,

(2) $\operatorname{deg}_{C_{i}^{\prime \prime}} L^{\prime}=d_{i}$, that is, the multidegree of $L^{\prime}$ with respect to this decomposition is equal to $\underline{d}$.

Proof. If $\left(X^{\prime}, L^{\prime}\right)$ satisfies the above condition, we will explicitly construct a polarized, one-parameter deformation $\left(\mathscr{Z}^{\prime}, \mathscr{L}^{\prime}\right) \rightarrow\left(B, b_{0}\right)$ of $\left(X^{\prime}, L^{\prime}\right)$ to $X$, such that $X_{b} \cong X, \forall b \neq b_{0}$, and such that $\operatorname{deg} L_{b}^{\prime}=\underline{d}$.

This construction will also show that $V_{x}^{d}$ is nonempty. In fact, to say that $V_{x}^{\underline{d}}$ is empty is to say that $\forall b \neq b_{0}, \operatorname{hilb}\left(X_{b}^{\prime}, L_{b}^{\prime}\right)$ is unstable. But this would imply that $h^{\prime}=\operatorname{hilb}\left(X^{\prime}, L^{\prime}\right)$ is also unstable, as the locus of unstable points is closed. This contradicts the assumption that $h^{\prime}$ be $G$-semistable.

Notice that $h^{\prime}$ determines the line bundle $L^{\prime}$ only up to the action of $\operatorname{Aut}\left(X^{\prime}\right)$; in fact we will really consider $L^{\prime}$ modulo automorphisms of $X^{\prime}$.

We will exhibit the construction in the case that $X^{\prime}$ has a unique new destabilizing component, that is, $\rho=1$; the general case is obtained by iterating (notice that the fact that $h^{\prime}$ is semistable implies that $E_{i} \cap E_{j}=\varnothing \forall i, j$ ).

The idea for constructing such a family comes from a work by Gieseker [G2].

Let $E$ be the new destabilizing component of $X^{\prime}$; then we write $X^{\prime}=E \cup$ $X^{\nu}$, where $X^{\nu}$ denotes the partial normalization of $X$ at the node $q$ generated by contracting $E$. We can assume that $E \cap C_{1}^{\prime} \neq \varnothing$ and that $C_{1}^{\prime \prime}=C_{1}^{\prime} \cup$ 
$E, C_{i}^{\prime \prime}=C_{i}, \forall i \geq 2$, in particular, $C_{1}^{\prime}$ is either isomorphic to $C_{1}$ or equal to the partial normalization of $C_{1}$ at $q$.

If $\nu: X^{\nu} \rightarrow X$ is the (partial) normalization map, let $\nu^{-1}(q)=\left\{p_{1}, p_{2}\right\}$, with $p_{1} \in C_{1}$. Since $L^{\prime}$ must satisfy the Basic Inequality with respect to $X^{\prime}$, $\operatorname{deg} L_{\mid E}^{\prime} \leq 1$; if $\operatorname{deg} L_{\mid E}^{\prime}=0, X=X^{\prime}$ and there is nothing to prove; hence $\operatorname{deg} L_{\mid E}^{\prime}=1$. Let now $L^{\nu} \in \operatorname{Pic}_{X^{\nu}}^{d-1}$ denote the restriction of $L^{\prime}$ to $X^{\nu} ; p_{1}$ is a regular point of $X^{\nu}$, therefore there exists a unique $M \in \mathrm{Pic}_{X^{\nu}}^{d}$ such that $M=L^{\nu} \otimes \mathscr{O}_{X^{\nu}}\left(p_{1}\right)$.

Now consider the family $\mathbb{P}^{1} \times_{k} X^{\nu}$ over $\mathbb{P}^{1}$ and the line bundle $\mathscr{M}=p_{X^{\nu}}^{*} M$ on it.

If we pick any point $b_{0}$ on $\mathbb{P}^{1}$, we can find an isomorphism $\alpha: \mathscr{M}_{2} \rightarrow \mathscr{M}_{1} \otimes$ $\mathscr{O}_{B_{1}}\left(-b_{0}\right)$, defined in an open neighborhood $B$ of $b_{0}$, where $B_{i}=B \times\left\{p_{i}\right\}$, $i=1,2$, and let $\mathscr{M}_{i}$ denote the restriction of $\mathscr{M}$ to $B_{i}$. Let $\mathscr{Z}=B \times X^{\nu}$.

Let now $\sigma: \mathscr{Y} \rightarrow \mathscr{Z}$ be the blow up of the surface $B \times X^{\nu}$ at the point $\left(b_{0}, p_{1}\right)$, and let $E$ be the exceptional divisor. On $\mathscr{Y}$, we can define a line bundle

$$
\mathscr{M}^{\prime}=\sigma^{*} \mathscr{M} \otimes \mathscr{O}_{\mathscr{Y}}(-E)
$$

which has the property that, over a neighborhood of $b_{0}, \mathscr{M}_{\mid B_{1}^{\prime}}^{\prime} \cong \mathscr{M}_{\mid B_{2}^{\prime}}^{\prime}$, via $\alpha$ ( $B_{i}^{\prime}$ is the proper transform of $B_{i}$ ).

Let $\mathscr{X}^{\prime} \rightarrow U$ be the new family obtained by fiberwise gluing $B_{1}^{\prime}$ to $B_{2}^{\prime}$; its fiber over $b_{0}$ is isomorphic to $X^{\prime}$, and every other fiber is isomorphic to $X$; moreover, we can use $\alpha$ to descend $\mathscr{M}^{\prime}$ to a line bundle $\mathscr{L}^{\prime}$ on $\mathscr{X}^{\prime} . \mathscr{L}^{\prime}$ restricts to $L^{\prime \prime}$ on $X^{\prime}$, and clearly $L^{\prime \prime}$ differs from $L^{\prime}$ by an automorphism of $X^{\prime}$. It is clear from the construction that $\underline{d}$ is the multidegree of the restriction of $\mathscr{L}^{\prime}$ to $X$.

To conclude, we need to show that the canonical map $B \longrightarrow$ Hilb $_{r}^{p(x)}$ maps $B \backslash\left\{b_{0}\right\}$ in $H_{d}$ (we may have to shrink $B$ around $b_{0}$ for that). But, as we already mentioned, this follows from the assumption that $\operatorname{hilb}\left(X^{\prime}, L^{\prime}\right)$ is semistable and from the fact that the locus of unstable points is closed in Hilb $_{r}^{p(x)}$.

The converse is much simpler to show. Let $h^{\prime} \in V_{x}^{\underline{d}}$. Then $X^{\prime}$ is clearly obtained by destabilizing $\rho$ nodes of $X$, and we can decompose $X^{\prime}$ as in the statement of the proposition,

$$
X^{\prime}=\left(\cup_{1}^{\gamma} C_{i}^{\prime}\right) \cup\left(\cup_{1}^{\rho} E_{j}\right)
$$

and

$$
\underline{d}^{\prime}=\left(d_{1}^{\prime}, \ldots, d_{\gamma}^{\prime}, 1, \ldots, 1\right) .
$$

If we consider any curve $B \subset V_{x}^{\underline{d}}$ such that $h^{\prime} \in B$ and $B \backslash\left\{h^{\prime}\right\} \subset M_{x}^{\underline{d}}$, then the restriction of the universal curve over $\mathrm{Hilb}_{r}^{p(x)}$ gives us a polarized family $\left(\mathscr{L}^{\prime}, \mathscr{Z}^{\prime}\right)$ over $B$ whose general fiber is isomorphic to $X$ and whose fiber over 
$h^{\prime}$ is $X^{\prime}$. We can assume that $B$ is smooth; the total space $\mathscr{Z}^{\prime}$ will be singular along those curves which correspond to the nodes of $X$.

Let $\mathscr{Y}$ be the new family of curves obtained by normalizing $\mathscr{Z}^{\prime}$ along all curves corresponding to those nodes of $X$ which belong to two different irreducible components.

$\mathscr{Y}$ will have $\gamma$ connected components, $\mathscr{Y}_{1}, \ldots, \mathscr{Y}_{y}$. The general fiber of $\mathscr{Y}_{i}$ will be $C_{i}$. We will define $C_{i}^{\prime \prime}$ to be the fiber of $\mathscr{Y}_{i}$ over $h^{\prime}$. By pulling back $\mathscr{L}^{\prime}$ to $\mathscr{Y}_{i}$ we see that $C_{i}^{\prime \prime}$, the required condition, is satisfied.

In particular, every irreducible component of $\psi_{d}^{-1}([X])$ contains a dense, open subset consisting of Hilbert points of projective embeddings of $X$.

Example. Let us consider a DM-stable curve $X$ having only two irreducible smooth components: $X=C_{1} \cup C_{2}$, with $\left|C_{1} \cap C_{2}\right|=k$. Suppose $\underline{d}=\left(d_{1}, d_{2}\right)$ is such that $M_{x}^{\underline{d}}$ is not empty. If $h^{\prime}=\operatorname{hilb}\left(X^{\prime}, L^{\prime}\right) \in V_{x}^{\underline{d}}$, then the proposition says that

$$
X^{\prime}=C_{1}^{\prime} \cup C_{2}^{\prime} \cup\left(\cup_{j=1}^{\rho} E_{j}\right)
$$

with $\left|C_{1}^{\prime} \cap C_{2}^{\prime}\right|=k-\rho$ and $C_{i} \equiv C_{i}^{\prime}, \quad i=1,2$. Moreover, the condition on $\underline{d}^{\prime}$ is simply

$$
d_{i}-\rho \leq d_{i}^{\prime} \leq d_{i}, \quad i=1,2 .
$$

If $\rho=1$ we have the situation depicted in Figure 2 and there are only two possibilities for $\underline{d}^{\prime}$ : either

$$
\underline{d}^{\prime}=\left(d_{1}-1, d_{2}, 1\right) \text { or } \underline{d}^{\prime}=\left(d_{1}, d_{2}-1,1\right) .
$$

In Figure 2 we have the latter possibility; in such a case the deformation of $X^{\prime}$ is locally trivial at $C_{1}^{\prime} \cap E_{1}$ and the "horizontal" curve represents a singular curve of the total space of the family.

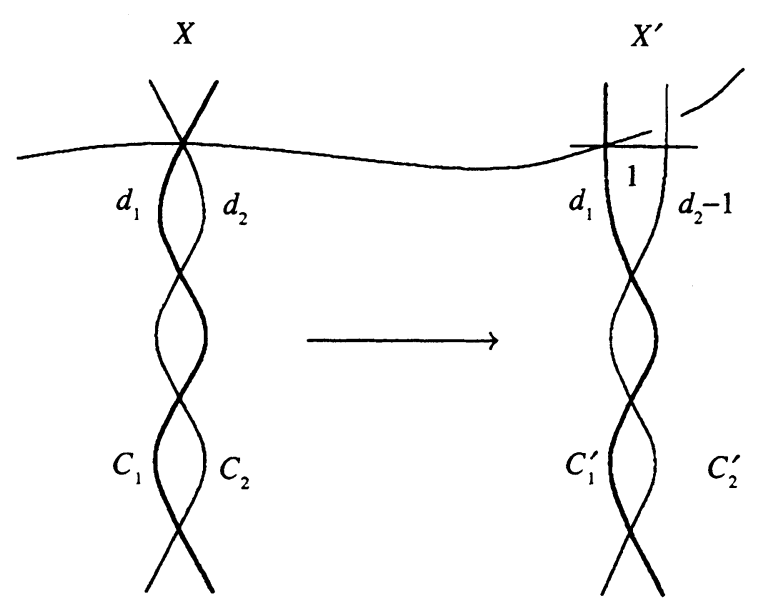

FIGURE 2 
Viceversa, if $h^{\prime}$ is a semistable point such that its corresponding curve is isomorphic to $X^{\prime}$, and $\underline{d}^{\prime}=\left(d_{1}^{\prime}, d_{2}^{\prime}, 1\right)$, then we have that $h^{\prime}$ lies in the intersection of $V_{\bar{X}}^{d^{-}}$and $V_{\bar{X}}^{d^{+}}$, where $X$ is the stable model of $X^{\prime}, \underline{d}^{+}=$ $\left(d_{1}^{\prime}+1, d_{2}^{\prime}\right)$, and $\underline{d}^{-}=\left(d_{1}^{\prime}, d_{2}^{\prime}+1\right)$.

5.1.1. Important remark (Degenerations in a fixed orbit). The family $\left(\mathscr{X}^{\prime}, \mathscr{L}^{\prime}\right)$ constructed in the proof of 5.1 induces a family of line bundles on $X$, which will not, in general, be constant; in fact the isomorphism $\alpha$ will have the effect of changing the gluing data, as the fiber varies.

There are special cases in which we can make such a family of line bundles constant (up to automorphisms of $X$ ). In other words, we will now describe a situation in which $h^{\prime} \in \overline{O_{G}(h)}$.

Let $X^{\prime}$ be a ladder, $X^{\prime}=Y^{\prime} \cup Z^{\prime} \cup D^{\prime}$, with $Y^{\prime} \cap Z^{\prime}=\varnothing$ and $D^{\prime}$ a union of $k$ destabilizing components, each of which meets both $Y^{\prime}$ and $Z^{\prime}$. Let $X=$ $Y \cup Z \cup D$ be obtained by contracting some of the destabilizing components of $D^{\prime}$ so that $Y \cong Y^{\prime}, Z \cong Z^{\prime}$, and $Y \cap Z=k-|D|$. Let $L \in \mathrm{Pic}_{X}^{\frac{d}{X}}$ be a line bundle with $\underline{d}=\left(m_{Y}, M_{Z}-|D|, 1, \ldots, 1\right)$. Then we claim that there exists a line bundle $L^{\prime}$ on $X^{\prime}$ having multidegree

$$
\underline{d}^{\prime}=\left(m_{Y}, m_{Z}, 1, \ldots, 1\right)
$$

such that

$$
\operatorname{hilb}\left(X^{\prime}, L^{\prime}\right) \in \overline{O_{G}(\operatorname{hilb}(X, L))} \text {. }
$$

Viceversa, given $L^{\prime}$ on $X^{\prime}$ as above, we can show that there exists an $L \in$ $\operatorname{Pic}_{X}^{d}$ such that the same conclusion holds.

To simplify the notation, let us first suppose that $X$ is DM-stable, so that $D$ is empty. We will show that the family $\left(\mathscr{Z}^{\prime}, \mathscr{L}^{\prime}\right)$ can be constructed so that the polarized fiber over every $b \neq b_{0}$ is isomorphic to $(X, L)$.

Suppose we are given $L \in \mathrm{Pic}_{X}^{\frac{d}{X}}$ as above. We can perform the same degeneration construction of the proof of the first part of 5.1, simultaneously at all nodes $\left\{q^{1}, \ldots, q^{k}\right\} \subset X$ lying on $Y \cap Z$. Namely, let $\nu: X^{\nu} \rightarrow X$ be the normalization at the $q^{j}$ and let $p_{1}^{j}$ (respectively $p_{2}^{j}$ ) be the point in $Z$ (respectively $Y$ ) which is mapped to $q^{j}$ by $\nu$. $\mathscr{M}$ will now be the pull-back of $L$ to $B \times X^{\nu}$ and we will fix an isomorphism for each $j$

$$
\alpha^{j}: \mathscr{M}_{2}^{j} \rightarrow \mathscr{M}_{1}^{j}\left(-b_{0}\right)
$$

such that the $\alpha^{j}$ act in the same way, on each fiber over $b \neq b_{0}$. These gluing data will simply have the effect of applying an automorphism to the polarization of the general fiber, that is: $L_{B}^{\prime} \cong L$ for all $b \neq b_{0}$.

The proof of the converse follows by applying analogous modifications to the deformation construction in the proposition.

Finally, if $X$ is not DM-stable, then the degree of freedom given by $X^{\nu}$ being disconnected will not be available; what we can use in this case is the nonlinear action of the automorphisms of $X$, on the varying gluing data. The conclusion will still be that $h^{\prime}$ lies in the orbit closure of $h$. 


\subsection{GIT-semistable ladders.}

Definition. (1) Let $X$ and $X^{\prime}$ be two DM-semistable curves; we say that $X^{\prime}$ dominates $X$ if they have the same stable model and if there exists a surjective morphism of $X^{\prime}$ onto $X$ under which every component of $X^{\prime}$ is either contracted to a point or mapped birationally onto its image.

(2) Let $\underline{d} \in B_{X}$ and $\underline{d}^{\prime} \in B_{X^{\prime}}$; we say that $\underline{d}^{\prime}$ is a refinement of $\underline{d}$, in symbols

$$
\underline{d}^{\prime} \preceq \underline{d},
$$

if and only if the condition in Proposition 5.1 is satisfied. This can be equivalently stated as follows:

$\underline{d}^{\prime} \preceq \underline{d}$ if and only if $X^{\prime}$ dominates $X$ via $\sigma$ and for every subcurve $Y$ of $X$ there exists a subcurve $Y^{\prime}$ of $X^{\prime}$ such that $\sigma$ maps $Y^{\prime}$ onto $Y$ and $d_{Y}=d_{Y^{\prime}}$.

We will also say that $X^{\prime}$ is a refinement of $X$, when there will be no ambiguity on the multidegrees.

(3) Let $X^{\prime}$ dominate $X$ via a surjective morphism $\sigma$, let $L$ be a line bundle of multidegree $\underline{d}$ on $X$, and let $\underline{d}^{*}$ be defined as

$$
\underline{d}^{*}=\underline{\operatorname{deg}} \sigma^{*} L \text {. }
$$

In such a case we say that $\underline{d}^{*}$ is the pull-back of $\underline{d}$ to $X^{\prime}$.

(4) Let $X$ be quasistable and let $\underline{d}$ be a positive multidegree on it, such that $\underline{d}$ satisfies $(\mathrm{BI})$. We say that $(X, \underline{d})$ is extremal if and only if for every subcurve $Y$ of $X$ such that $d_{Y}=m_{Y}$, we have

$$
Y \cap(\overline{X \backslash Y}) \subset D_{X},
$$

in other words, no node of $Y \cap \overline{X \backslash Y}$ is stable (cf. 1.1).

In the GIT set-up, extremal curves will play a special role; cf. Lemma 6.1.

It is also worth noticing the following consequence of the Fundamental Theorem of GIT (cf. 1.2): let $h=\operatorname{hilb}(X, L)$ be in $H_{d}$; then there exists a unique curve $X^{\prime}$ with a unique (up to automorphisms of $X^{\prime}$ ) very ample line bundle $L^{\prime}$ such that $h^{\prime}=\operatorname{hilb}\left(X^{\prime}, L^{\prime}\right)$ is contained in the semistable closure of the orbit of $h$. In fact, every such orbit contains a unique closed orbit in its semistable closure. One expects $h^{\prime}$ to correspond to a suitable extremal ladder; this will follow from the next lemma.

We first observe that the statement is clear for curves whose stable model has two irreducible components.

5.2.1. Important remark. Suppose that $X=Y \cup Z \subset \mathbb{P}^{r}$ is a DM-stable curve which is the union of two smooth irreducible components; let $\underline{d}=\left(m_{Y}, M_{Z}\right)$ be its multidegree; assume that $h=\operatorname{hilb}(X)$ is semistable. Then on the (unique!) ladder $X^{\prime}$. such that $s t\left(X^{\prime}\right)=X$ there exists a line bundle $L^{\prime}$ of multidegree $\underline{d}^{\prime}=\left(m_{Y}, m_{Z}, 1, \ldots, 1\right)$ such that $h^{\prime}=\operatorname{hilb}\left(X^{\prime}, L^{\prime}\right)$ is contained in the closure of the orbit of $h$, and $h^{\prime}$ is itself semistable. Moreover, the orbit of $h^{\prime}$ is uniquely determined by the pull-back of $L$ to the normalization of $X$. 
In fact, by Lemma $3.1, h$ is strictly semistable; since its stabilizer is finite, $\overline{O_{G}(h)} \cap H_{d}$ must contain some orbit of smaller dimension. From Lemma 3.2 and Proposition 3.1 the observation follows. Observe also that if $X^{\prime \prime}$ is quasistable with $s t\left(X^{\prime \prime}\right)=X$, and if $\underline{d}=\left(m_{Y}, M_{Z}-u, 1, \ldots, 1\right)$ so that $X^{\prime \prime}$ has $u$ destabilizing components, then exactly the same conclusion holds.

Now in general we have

Lemma 5.1. Let $X \subset \mathbb{P}^{r}$ be quasistable of multidegree $\underline{d}$; assume that there exists a subcurve $Y$ of $X$ for which $d_{Y}=m_{Y}$. If $h=\operatorname{hilb}(X)$ is semistable, there exists $h^{\prime}$,

$$
h^{\prime} \in \overline{O_{G}(h)} \cap H_{d},
$$

with the following property:

$h^{\prime}=\operatorname{hilb}\left(X^{\prime}\right)$ where $X^{\prime} \subset \mathbb{P}^{r}$ is a ladder of multidegree $\underline{d}^{\prime}$, which dominates $X$ via the morphism

$$
\sigma: X^{\prime} \longrightarrow X
$$

and such that

$$
\underline{d}^{\prime} \preceq \underline{d} ;
$$

moreover $\sigma$ destabilizes all the stable nodes of $Y \cap Z$, and no other node of $X$.

Cf. Section 1.1 for the terminology we use in the statement. As usual, $Z$ denotes the complementary curve of $Y$ in $X$.

Proof. Of course, if $Y \cap Z \subset D_{X}$, we have nothing to prove, for $X$ itself is a ladder of the type we want. Furthermore if $Y$ and $Z$ are smooth and irreducible, our lemma has been proved in 5.2.1.

We start by considering a deformation $\pi:(\mathscr{Z}, \mathscr{L}) \longrightarrow\left(B, b_{0}\right)$ of $(X, L)$ (where $L:=\mathscr{O}_{X}(1)$ ), which is locally trivial at $Y \cap Z$ and smoothing all the remaining nodes of $X$. The general fiber $X_{b}$ will therefore be

$$
X_{b}=Y_{b} \cup Z_{b}
$$

with $Y_{b}$ and $Z_{b}$ smooth and irreducible (see the end of the proof of 3.1 for the reduction to $Y$ and $Z$ connected).

By hypothesis, $\operatorname{hilb}(X, L)$ is semistable, hence $\operatorname{hilb}\left(X_{b}, L_{b}\right)$ will also be semistable for $b$ in an open neighborhood of $b_{0}$. We can assume that $B \subset H_{d}$ and that $(\mathscr{Z}, \mathscr{L})$ is the restriction of the universal family. We can also assume that over $B$ we have a section of the canonical map

$$
W \backslash\{0\} \rightarrow \mathbb{P}(W) \supset \operatorname{Hilb}_{r}^{p(x)}
$$

(cf. Section 1.2); that is, we have a continuous choice of vectors $v_{b}$ representing $b$.

In Lemma 3.1 we proved that $b$ is strictly semistable by exhibiting a oneparameter subgroup $\lambda_{b}(t)$ such that

$$
\lim _{t \rightarrow \infty} \lambda_{b}(t) \cdot v_{b}=v_{b}(\infty) \neq 0 .
$$


Such a $\lambda_{b}(t)$ was constructed starting from the filtration

$$
0 \subset U_{b} \subset V=H^{0}\left(\mathbb{P}^{r}, \mathscr{O}_{\mathbb{P}^{r}}(1)\right)
$$

with

$$
U_{b}:=\operatorname{Ker}\left\{H^{0}\left(\mathbb{P}^{r}, \mathscr{O}_{\mathbb{P}^{r}}(1)\right) \longrightarrow H^{0}\left(Y_{b}, \mathscr{O}_{Y_{b}}(1)\right)\right\}
$$

together with a relative basis (cf. the proof of Proposition 3.1). Now we can easily define these $\lambda_{b}$ in such a way that they vary in a nice family parametrized by $B$. To do that, let $V \times_{k} B$ be the trivial bundle over $B$ and let

$$
U:=\operatorname{Ker}\left\{V \times_{k} B \longrightarrow\left(\pi_{\mathscr{y}}\right)_{*} \mathscr{L}_{\mathscr{y}}\right\}
$$

where $\mathscr{L}_{\mathscr{y}}$ (resp. $\pi_{\mathscr{y}}$ ) is the pull-back of $\mathscr{L}$ (resp. $\pi$ ) to the irreducible component of $\mathscr{X}$ having $Y_{b}$ as fiber. $\left(\pi_{\mathscr{y}}\right)_{*} \mathscr{L}_{\mathscr{y}}$ is clearly a vector bundle over $B$, hence $U$ is a vector subbundle of $V \times_{k} B$. We can now chose a frame for $U$ (modulo shrinking $B$ ) in such a way that we can define the $\lambda_{b}$ continuously over $B$.

We know that, for all $b \in B, v_{b}(\infty)$ corresponds to a semistable point having positive dimensional stabilizer; moreover, by 5.2.1, we know that, for all $b \neq b_{0}, v_{b}(\infty)$ corresponds to a ladder of the type we want. By construction, these ladders form a polarized family $\left(\mathscr{C}^{\prime}, \mathscr{L}^{\prime}\right)$ parametrized by $B$. We have to show that the central fiber satisfies the condition in the lemma.

It is clear that $\left(X^{\prime}, L^{\prime}\right)$ contains the required destabilizing components, because so does the general fiber; moreover $\left(X^{\prime}, L^{\prime}\right)$ is a refinement of $X$, by Proposition 5.1.

Finally, we have to show that $X^{\prime}$ contains no unnecessary destabilizing components. Now, to say that there is an $E$ which does not appear in $X$ and which is not a specialization of a destabilizing component in the general fiber, is to say that the line bundle $L_{Z}(-D)$ has some base points, where we defined $D$ to be the divisor of $Z$ corresponding to $Z \cap Y$.

To conclude, we will show that, if $L_{Z}(-D)$ has a base point $p$, then $h$ will not be semistable. By arguing as in the proof of Proposition 3.1 we find that, if $p$ is a base point of $L_{Z}(-D)$, the following inequality holds

$$
b_{i} \leq h^{0}\left(Z, L_{Z}^{m}((i-m) D-p)\right)
$$

which gives, together with the relation $(*)$ in the proof of Lemma 3.1, $T \geq m^{2}\left(d_{Y}+k / 2+1\right)+O(m)>m^{2} \frac{h^{0}\left(Y, L_{Y}\right)}{h^{0}(X, L)} d+O(m) \geq \frac{\sum_{0}^{r} w_{j}}{h^{0}(X, L)} m p(m) ;$ hence, by the numerical criterion, $h$ is not semistable.

5.3. A suitable separation property for polarized families of semistable curves. "Suitable" here means that polarizations are considered modulo "twists".

The next lemma will be used as a bridge between the semistable replacement property of GIT and our combinatorial results. 
Lemma 5.2. Let $(\mathscr{X}, \mathscr{L})$ and $(\mathscr{Y}, \mathscr{M})$ be two generic, locally trivial oneparameter families of $D M$-semistable curves over a smooth, pointed base $\left(B, b_{0}\right)$. Assume that over $B \backslash\left\{b_{0}\right\}$ there exists an isomorphism between the two polarized families.

Then there exists a family of DM-semistable curves

$$
\mathscr{W} \longrightarrow\left(B, b_{0}\right)
$$

having a birational morphism onto both $\mathscr{X}$ and $\mathscr{Y}$, which is biregular away from $b_{0}$, and such that the pull-backs of $\mathscr{L}$ and $\mathscr{M}$ to $\mathscr{W}$ differ by a Cartier divisor supported on the central fiber.

Proof. First of all notice that, by the properness of DM-stable curves, $\mathscr{Z}$ and $\mathscr{Y}$ have the same stable reduction; hence there exists a family $\mathscr{Z}$ of DMsemistable curves which maps to both $\mathscr{X}$ and $\mathscr{Y}$ isomorphically away from the central fiber. The pull-backs of $\mathscr{L}$ and $\mathscr{M}$ to $\mathscr{Z}$ will be isomorphic away from $b_{0}$; more precisely, such an isomorphism comes together with a family of automorphisms of the fibers, which need not have a limit over $b_{0}$. In other words, all that we know is that there exists a birational map

$$
\tau: \mathscr{Z} \rightarrow \mathscr{Z}
$$

which is biregular away from the central fiber, where we have

$$
\tau^{*} \mathscr{L}=\mathscr{M}
$$

For example, if the general fiber were DM-stable, such a family of automorphisms would be trivial and it would be enough to take $\mathscr{W}=\mathscr{Z}$; in fact there will be an indeterminacy problem only if the general fiber has a continuous automorphism group. A more enlightening example is described after the lemma.

To solve this difficulty we introduce a new piece of information, which takes automorphisms into account. By. what we said, the only automorphisms that will play a role are the ones coming from the destabilizing components, hence we can do what follows. Let $\mathscr{E} \rightarrow B$ be an irreducible component of $\mathscr{X}$ such that $\mathscr{E} \cong \mathbb{P}^{1} \times_{k} B$ and has precisely two attaching sections. Let $\mathscr{E}^{\prime}$ be the corresponding component of $\mathscr{Z}$. We can take a section $S$ of $\mathscr{E}$ which does not meet the two attaching sections over the central fiber. Let now $S^{\prime}$ be the closure of the preimage of $S$ in $\mathscr{Z}$ under the family of automorphisms defined by $\mathscr{L}$ and $\mathscr{M}$, that is,

$$
S^{\prime}=\overline{\tau^{-1} \sigma^{-1}\left(S_{\mid B \backslash\left\{b_{0}\right\}}\right)},
$$

$\sigma$ being the map from $\mathscr{Z}$ to $\mathscr{X}$. To say that such a family of automorphisms does not have a limit is to say that $S^{\prime}$ meets the attaching sections over the central fiber.

To resolve such an indeterminacy it will be enough to blow up $\mathscr{E}^{\prime}$ until the proper transform of $S^{\prime}$ meets the central fiber away from the attaching points. 
We let $\mathscr{W}$ be the family obtained after applying this process to every such $\mathscr{E} \subset \mathscr{Z}$. It is clear that all we have done is to introduce new destabilizing chains in the central fiber, hence $\mathscr{W}$ satisfies the requirements in the lemma.

Example. In Figure 3, we consider families whose general fiber is a quasistable curve $X=C_{1} \cup C_{2} \cup C_{3}$ with $\left|C_{i} \cap C_{j}\right|=1$ if $i \neq j$ and such that $C_{3}$ is isomorphic to $\mathbb{P}^{1}$. We also assume for simplicity that the two remaining components have the same genus, so that $m_{C_{1}}=m_{C_{2}}=m$.

The three families $\mathscr{Z}, \mathscr{Z}$, and $\mathscr{W}$ in the lemma are pictured by the horizontal arrows. We take $(\mathscr{Z}, \mathscr{L})$ to be trivial, that is, $\mathscr{Z}=X \times{ }_{k} B$ and $\mathscr{L}=p_{X}^{*} L$, with $\operatorname{deg} L=(m, m+1,1)$. ( $\mathscr{Z}, \mathscr{M})$ is a degeneration of $(X, L)$ to $(Z, M)$ such as the ones described in 5.1.1.

Just by looking at the degree class group of $Z$, one sees that, although they are isomorphic away from the central fiber, $\mathscr{L}$ and $\mathscr{M}$ do not differ by a Cartier divisor of $\mathscr{Z}$ supported on the central fiber. (There are no such Cartier divisors at all in $\mathscr{Z}$.) What happens is that $\tau$ contracts $F$ to a point.

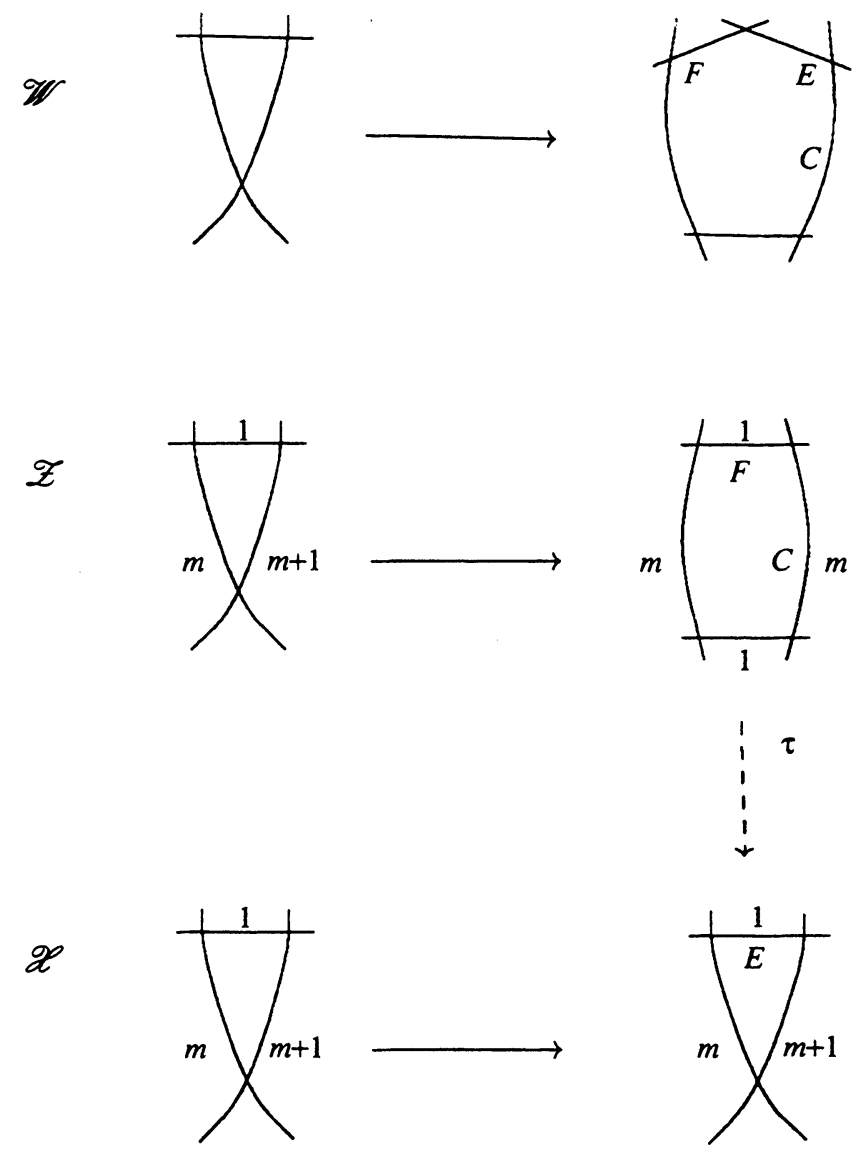

FIGURE 3 
We obtain $\mathscr{W}$ by blowing up the point corresponding to $F \cap C$; finally we have

$$
\mathscr{M}^{*}=\mathscr{L}^{*} \otimes \mathscr{O}_{\mathscr{W}}(E+C)
$$

5.4. A completeness result. The central part of the argument used to prove the next proposition will also give as a result Lemma 6.1 .

Proposition 5.2. Let $X$ be a quasistable curve. If there exists $F \in \mathrm{Pic}_{X}^{\frac{d}{}}, F$ very ample, such that $\operatorname{hilb}(X, F)$ is semistable, then for every $L \in \operatorname{Pic}_{X}^{\frac{d}{X}}, \operatorname{hilb}(X, L)$ is semistable.

Proof. First of all, notice that the subset of $\mathrm{Pic}_{X}^{\frac{d}{X}}$ of line bundles having semistable Hilbert point is a dense open subset. In fact, let $\mathscr{P}$ be any Poincaré line bundle on $\operatorname{Pic}_{X}^{\frac{d}{2}} \times_{k} X$; then its push-forward to $\operatorname{Pic}_{X}^{\frac{d}{X}}$ is a vector bundle $E$ of rank $r+1$. We can therefore consider the irreducible variety $P G L(E)$ over $\operatorname{Pic}_{X}^{d}$.

Clearly $P G L(E)$ maps to $\mathrm{Hilb}_{r}^{p(x)}$; therefore the claim follows from the fact that the semistable points of $\mathrm{Hilb}_{r}^{p(x)}$ form an open subset.

If $L \in \operatorname{Pic}_{X}^{d}$, then $h=\operatorname{hilb}(X, L)$ lies in the closure of $M_{X}^{\frac{d}{d}}$ in $\mathrm{Hilb}_{r}^{p(x)}$. This allows us to consider a deformation $(\mathscr{X}, \mathscr{L})$ of $(X, L)$ over a smooth one-dimensional base $\left(B^{\prime}, b_{0}^{\prime}\right)$ such that the associated map to $\mathrm{Hilb}_{r}^{p(x)}$ maps $B^{\prime} \backslash\left\{b_{0}^{\prime}\right\}$ to $M_{x}^{\underline{d}}$ and $b_{0}^{\prime}$ to $h$.

Assume that $h$ is not semistable; then the semistable replacement property (cf. 1.2.1) implies that on a ramified cover $B$ of $B^{\prime}$, we can construct a new family $(\mathscr{Y}, \mathscr{M}) \longrightarrow B$, which maps to $H_{d}$ by sending $b \in B$ to $\operatorname{hilb}\left(Y_{b}, M_{b}\right)$. Moreover, if we denote again by $(\mathscr{X}, \mathscr{L})$ the fiber product over $B$ of the initial family, for all $b \neq b_{0}, \operatorname{hilb}\left(Y_{b}, M_{b}\right)$ is $G$-equivalent to $\operatorname{hilb}\left(X_{b}, L_{b}\right)$. Let $M$ be the restriction of $\mathscr{M}$ to the central fiber $Y \cong X^{\prime}$ of $\mathscr{Y}$, let $\underline{d}^{\prime}=\operatorname{deg} M$, and let $h^{\prime}=\operatorname{hilb}\left(X^{\prime}, M\right)$ be just $b_{0}$; it is clear that $h^{\prime} \in V_{x}^{\underline{d}}$, that is, $\underline{d}^{\prime}$ is a refinement of $\underline{d}$.

$(\mathscr{Y}, \mathscr{M})$ and $(\mathscr{X}, \mathscr{L})$ are isomorphic away from their fibers over $b_{0}$ and the properness property of stable curves implies that the stable reduction of $\mathscr{Z}$ is isomorphic to the stable reduction of $\mathscr{Y}$. Therefore the two families will be generic locally trivial, away from some rational double points lying at the nodes of the central fibers; these isolated singularities can be resolved by adding some destabilizing chains. After performing the above operation, we will be under the hypothesis of Lemma 5.2 , hence there exists a family $\mathscr{W} \longrightarrow\left(B, b_{0}\right)$ dominating both $\mathscr{X}$ and $\mathscr{Y}$ and such that we have

$$
\mathscr{L}^{*} \cong \mathscr{M}^{*} \otimes \mathscr{O}_{\mathscr{W}}(T)
$$

where $\mathscr{L}^{*}$ and $\mathscr{M}^{*}$ denote the pull-backs to $\mathscr{W}$ and $T$ is a Cartier divisor supported on $W$. 
Let $\underline{d}^{*}=\underline{\operatorname{deg}} \mathscr{L}_{\mid W}^{*}$ and $\underline{d}^{\prime \prime}=\underline{\operatorname{deg}} \mathscr{M}_{\mid W}^{*} ;$ by Lemma 4.1 we can write in normal form

$$
\underline{d}^{*}-\underline{d}^{\prime \prime}=\sum_{l=0}^{q-1} \underline{b_{l}^{\prime}}
$$

where the $B_{l}^{\prime}$ play here the role of the $B_{l}$ in 4.1.

Let $\sigma: \mathscr{W} \longrightarrow \mathscr{X}$ be the canonical map, and let us denote $B_{l}:=\sigma\left(B_{l}^{\prime}\right)$ and $A_{l}:=\sigma\left(A_{l}^{\prime}\right)$.

We will now prove the proposition under the assumption that the pair $(X, L)$ is extremal (cf. Definition in 5.2).

We know that $\underline{d}^{\prime} \preceq \underline{d}$. Suppose $\underline{d}^{\prime} \neq \underline{d}$; then there exists a destabilizing component $E \subset W$ such that $d_{E}^{*}=0, d_{E}^{\prime \prime}=1$, and $\sigma(E)$ is a stable node of $X$. In other words, $E$ appears in $X^{\prime}$ but not in $X$.

By Corollary 4.1 , there exists an $l$ such that $E \subset B_{l}^{\prime}$ and $\left|E \cap A_{l}^{\prime}\right|=1$. But now,

$$
d_{B_{l}}=d_{B_{l}^{\prime}}^{*}=m_{B_{l}^{\prime}}=m_{B_{l}}
$$

and, since $X$ is extremal, $\sigma(E)$ cannot possibly be a stable node.

We conclude that $\underline{d}=\underline{d}^{\prime}$, hence, for every irreducible component $C$ of $W$ such that $C$ is not destabilizing, we have

$$
\mathscr{L}_{\mid C}^{*} \cong \mathscr{M}_{\mid C}^{*}
$$

We therefore obtain that the Hilbert points of the two projective models of $W$, determined by $\mathscr{L}_{\mid C}^{*}$ and $\mathscr{M}_{\mid C}^{*}$, have the same orbit (cf. 5.2.1). That is to say that $\operatorname{hilb}(X, L) \in O_{G}\left(\operatorname{hilb}\left(X^{\prime}, M\right)\right)$, from which we immediately conclude that $\operatorname{hilb}(X, L)$ is semistable, as $\operatorname{hilb}\left(X^{\prime}, M\right)$ is. This is a contradiction.

Now that we proved the proposition for extremal curves, we can easily deal with the general case.

By Lemma 5.1, for any semistable $\operatorname{hilb}(X, F)$, with $F \in \operatorname{Pic}_{X}^{\frac{d}{X}}$, there exists an extremal ladder $X^{\ell}$ with a line bundle $F^{\ell}$ of multidegree $\underline{d}^{\ell}$, whose Hilbert point is semistable and such that, more precisely,

$$
\operatorname{hilb}\left(X^{\ell}, F^{\ell}\right) \subset \overline{O_{G}(\operatorname{hilb}(X, F))} \cap H_{d} .
$$

By the previous argument, we conclude that every line bundle of degree $\underline{d}^{\ell}$ on $X^{\ell}$ is GIT-semistable. Now, by 5.1 .1 given any $L \in \mathrm{Pic}_{X}^{\frac{d}{X}}$, there exists a line bundle $L^{\prime}$ of degree $\underline{d}^{\ell}$ on $X^{\ell}$ such that $\left(X^{\ell}, L^{\prime}\right)$ can be deformed to $(X, L)$. That is, $\operatorname{hilb}\left(X^{\ell}, L^{\prime}\right)$ is in the orbit closure of $\operatorname{hilb}(X, L)$.

Since the unstable points form a closed subset, the fact that $\operatorname{hilb}\left(X^{\ell}, L^{\prime}\right)$ is semistable implies that $\operatorname{hilb}(X, L)$ is semistable.

\subsection{More terminology.}

Definition. We say that a given multidegree $\underline{d}$ is semistable on $X$ if $M_{x}^{\underline{d}}$ is not empty. 
Now Proposition 5.1 can be briefly restated as follows:

$$
M_{x^{\prime}}^{\underline{d}^{\prime}} \subseteq V_{x}^{\underline{d}} \Longleftrightarrow \underline{d}^{\prime} \preceq \underline{d}
$$

and, in particular, we want to stress that

\subsubsection{If $\underline{d}^{\prime} \preceq \underline{d}$ and $\underline{d}^{\prime}$ is semistable, then $\underline{d}$ is semistable.}

Let $X$ be DM-stable. It is now clear that $M_{x}^{\underline{d}}$ is either empty, or it has a map onto some quotient of $\mathrm{Pic}_{X} \frac{d}{X}$ by a finite group. The fibers of this map will be isomorphic to $P G L(r+1)$. In particular $M_{x}^{\underline{d}}$ is irreducible and, when nonempty, it has dimension $r(r+2)+g$.

Corollary 5.1. For all $[X]$ in $\bar{M}_{g}$,

(1) $\psi_{d}^{-1}([X])=V_{X}$.

(2) $\frac{d}{P_{d, X}}$ has at most $\mu(X)$ irreducible components, all of dimension $g$.

Proof. The first part is clear, by Proposition 5.1. Now

$$
\overline{P_{d, X}}=V_{X} / G
$$

and $\overline{P_{d, X}}$ is a projective (hence separated) scheme. Every irreducible component of it contains an open subset which parametrizes line bundles of given multidegree, hence there is an injection from the set of its components into $B_{X}$ (cf. Definition in 4.1).

\section{ConCLuSIONS}

\subsection{End of the proof of Theorem 3.1.}

Proposition 6.1 (Basic Inequality: sufficient condition). Let $X \subset \mathbb{P}^{r}$ be a connected, nodal, nondegenerate curve of degree $d$ and genus $g$; assume that its multidegree $\underline{d}$ satisfies the Basic Inequality. Then the Hilbert point of $X$ is semistable.

Proof. We will prove the proposition by contradiction; let $L^{0}=\mathscr{O}_{\mathbb{P}^{r}}(1) \otimes \mathscr{O}_{X}$ and let us assume that $\operatorname{hilb}\left(X, L^{0}\right)$ is unstable. We will use the semistable replacement property 1.2 .1 . Consider a general deformation of $\left(X, L^{0}\right)$ in a polarized family of generically smooth curves over a one-dimensional regular scheme $B^{\prime}$; if $X$ is the fiber over $b_{0}^{\prime}, B^{\prime} \backslash\left\{b_{0}^{\prime}\right\}$ has a map $f^{\prime}$ to $H_{d}$ (modulo shrinking $B^{\prime}$ around $b_{0}^{\prime}$ ). Applying the semistable replacement property yields a ramified cover $B$ of $B^{\prime}$ with a map $f$ to $H_{d}$ which is congruent to $f^{\prime}$ modulo $G$.

That implies that, if $\left(\mathscr{X}, \mathscr{L}^{0}\right)$ is the pull-back to $B$ of the initial family and $\left(\mathscr{Y}, \mathscr{M}^{0}\right)$ is the pull-back of the universal polarized family over $H_{d}$, the two polarized families are isomorphic away from the central fibers. We can apply Lemma 5.2 and derive that there exists a family of DM-semistable curves $\mathscr{W} \longrightarrow\left(B, b_{0}\right)$ which dominates both $\mathscr{Z}$ and $\mathscr{Y}$. Notice in fact that $\mathscr{Z}$ and $\mathscr{Y}$ may be singular at the nodes of their central fiber, but, since $\mathscr{X}$ is the 
base change of a smooth family, such singularities will be rational double points which can be desingularized by adding destabilizing chains.

Let us denote by $\mathscr{L}$ and $\mathscr{M}$ the pull-back to $\mathscr{W}$ of $\mathscr{L}^{0}$ and $\mathscr{M}^{0}$ respectively; by Lemma 5.2 we have

$$
\mathscr{L} \cong \mathscr{M} \otimes \mathscr{O}_{\mathscr{W}}\left(\sum_{l=0}^{q} B_{l}\right)
$$

where the $B_{l}$ are (reduced) subcurves of the central fiber $W$. Moreover, by Lemma 4.1, we can pick the $B_{l}$ in such a way that $B_{j} \cap A_{i}=\varnothing$ for all $i<j$, where recall that $A_{l}$ was defined to be the complementary curve of $B_{l}$.

As usual we denote by $L$ and $M$ the restrictions to $W$ of the given polarizations. The line bundle $L$ (respectively $M$ ) determines a map of $W$ in $\mathbb{P}^{r}$ whose image is a quasistable curve that we will denote by $W^{L}$ (respectively $W^{M}$ ); clearly $W^{L}=X$. (As usual, we disregard projective automorphisms.) Let $h=\operatorname{hilb}\left(W^{L}\right)$ and $h^{\prime}=\operatorname{hilb}\left(W^{M}\right)$; by construction $h^{\prime}$ is $G$-semistable.

The first step is to show that there exists a ladder $X^{\ell} \subset \mathbb{P}^{r}$ having degree $\underline{d}^{\ell}$ such that $\underline{d}^{\ell}$ is a refinement of both $\underline{d}$ and $\underline{d}^{\prime}, \underline{d}^{\prime}$ being the multidegree of $W^{M}$.

Let $\underline{d}^{*}=\underline{\operatorname{deg} L}$ and $\underline{d}^{\prime \prime}:=\left(\underline{d}^{\prime}\right)^{*}=\underline{\operatorname{deg} M}$; we write in normal form

$$
\underline{d}^{*}-\underline{d}^{\prime \prime}=\sum_{l=0}^{q} \underline{b}_{l}
$$

We will construct $X^{\ell}$ step by step, by working on one twist at a time. Let us suppose that

$$
\underline{d}^{*}-\underline{d}^{\prime \prime}=\underline{b}_{0}
$$

and let us denote, for simplicity, $B=B_{0}$ and $A=A_{0}=\overline{W \backslash B}$; then $d_{A}^{\prime \prime}=m_{A}$ and $d_{B}^{*}=m_{B}$. We can decompose $W=A^{\prime} \cup D \cup B^{\prime}$ where $D$ is the union of those destabilizing components which had precisely one node in $A \cap B$, $A^{\prime}=\overline{A \backslash D}$, and $B^{\prime}=\overline{B \backslash D}$.

Let $W^{\prime}$ be the DM-semistable curve obtained from $W$ by blowing up the nodes of $A^{\prime} \cap B^{\prime}$; on $W^{\prime}$ we now construct any line bundle $N$ which is isomorphic to $L_{\mid A^{\prime}}$ on $A, M_{\mid B^{\prime}}$ on $B$, and to $\mathscr{O}(1)$ on the remaining destabilizing components. $N$ maps $W^{\prime}$ to a ladder $X^{\prime}$ in $\mathbb{P}^{r}$, and, by construction, the multidegree of such a ladder is a refinement of both $\underline{d}$ and $\underline{d}^{\prime}$.

Since, by Proposition 4.1, $A_{i} \cap B_{j}=\varnothing$ if $i<j$, we can iterate this construction to obtain the $X^{\ell}$ of degree $\underline{d}^{\ell}$ that we wanted.

The second step is to prove that from the fact that $h^{\prime}$ is $G$-semistable follows that $\underline{d}^{\ell}$ is semistable (cf. Definition in 5.5). For that, we claim that $\underline{d}^{\ell}$ is equal to the degree of a ladder which satisfies the conditions of Lemma 5.1. To see this, let

$$
\sigma: X^{\ell} \longrightarrow W^{M}
$$


be the canonical map (viewed as a morphism of abstract curves); then all we have to check is that if $E$ is a destabilizing component of $X^{\ell}$ then either

(a) $\sigma$ maps $E$ isomorphically to its image, or

(b) there exist subcurves $Y$ and $Z$ in $W^{M}$ having no common components and such that $\sigma(E) \in Y \cap Z, d_{Y}=m_{Y}, d_{Z}=M_{Z}$. But this follows from Corollary 4.1. In fact, if $\sigma(E)$ is a point, then, by construction, $E$ is either obtained by destabilizing a point in $A^{\prime} \cap B^{\prime}$, in which case (b) holds, or $E$ is such that $d_{E}^{*}=1$ and $d_{E}^{\prime \prime}=0$. But then, by Corollary 4.1 , we conclude that $E \subset D$, hence (b) is satisfied.

By Lemma 5.1, there exists a ladder of degree $\underline{d}^{\ell}$ which is in the semistable closure of the orbit of $h^{\prime}$; hence, by Proposition 5.2, $\underline{d}^{\ell}$ is semistable.

Finally, by 5.5 .1 applied to $\underline{d}^{\ell} \preceq \underline{d}$, we conclude that $\underline{d}$ itself is semistable. In particular $\operatorname{hilb}\left(X, L^{0}\right)$ is semistable, and this is a contradiction.

6.2. Description of the GIT-stable points. We will now prove that a curve $X$ in $\mathbb{P}^{r}$ is $G$-stable if and only if the only subcurves $Z$ of $X$ having maximum allowed degree are the union of destabilizing components. In particular, if $X$ is a DM-stable curve, then $X$ is $G$-stable if and only if its multidegree satifies the strict Basic Inequality.

Lemma 6.1. Let $X \subset \mathbb{P}^{r}$ be a quasistable curve of multidegree $\underline{d}$ such that $h=\operatorname{hilb}(X) \in H_{d}$. Then the orbit of $h$ is closed in the semistable locus if and only if $(X, \underline{d})$ is extremal. In particular, $h$ is $G$-stable if and only if the only subcurves $Z$ of $X$ such that $d_{Z}=M_{Z}$ are the union of destabilizing components.

Proof. If $(X, \underline{d})$ is not extremal, then by Lemma 5.1 the orbit of $h$ is not closed in the semistable locus.

Viceversa, suppose that $(X, \underline{d})$ is extremal. Let $L$ be the line bundle embedding $X$ in $\mathbb{P}^{r}$ and let $(\mathscr{Y}, \mathscr{M}) \longrightarrow\left(B, b_{0}\right)$ be a polarized one-parameter family of GIT-semistable curves whose general fiber is isomorphic to $(X, L)$. We want to prove that the central family is also isomorphic to $(X, L)$. We can argue exactly like we did in the proof of Proposition 5.2 for extremal curves, by comparing $(\mathscr{Y}, \mathscr{M})$ with the trivial family $(\mathscr{Z}, \mathscr{L})=\left(X \times_{k} B, p_{X}^{*} L\right)$.

For the second part of the statement, we just need to recall that a point $h \in \mathrm{Hilb}_{r}^{p(x)}$ is stable if and only if its orbit is closed in the semistable locus and its stabilizer is finite.

6.3. General properties of $\overline{P_{d, g}}$ and $\overline{P_{d, X}}$. The following lemma is really a corollary of Proposition 5.1 and Proposition 3.1:

Lemma 6.2. $H_{d}$ is irreducible.

Proof. We know that $H_{d}$ is nonsingular; therefore, we can equivalently prove that it is connected. $H_{d}$ maps surjectively to $\bar{M}_{g}$ and its fibers over the smooth curves are irreducible varieties of dimension $g+r(r+2)$. Hence, if $H_{d}$ were not connected, it would have a component $Z$ which would be mapped by $f_{d}$ to the boundary, $\bar{M}_{g} \backslash M_{g}$. Since $\bar{M}_{g} \backslash M_{g}$ has codimension 1 and $Z$, if not 
empty, has dimension $4 g-3+r(r+2)$ (by Lemma 2.2) the generic fiber of $Z$ over $\bar{M}_{g} \backslash M_{g}$ would have dimension $g+r(r+2)+1$, which we already know to be impossible. We conclude that $Z$ is empty, that is, $H_{d}$ is irreducible.

The next theorem summarizes the essential points of the previous discussion. Theorem 6.1. (1) The projective scheme $\overline{P_{d, g}}$ is reduced, irreducible, and CohenMacaulay. It contains the open, dense subset $\phi_{d}^{-1}\left(M_{g}^{0}\right) \cong P_{d, g}$.

(2) The restriction of $\phi_{d}$ to $\phi_{d}^{-1}\left(\bar{M}_{g}^{0}\right)$ is flat.

(3) $\forall[X] \in \bar{M}_{g}^{0}, \overline{P_{d, X}}$ is a reduced, connected, projective scheme having at most $\mu(X)$ irreducible components, all having dimension $g$. The smooth locus of $\overline{P_{d, X}}$ is isomorphic to the (disjoint) union of a finite number of copies of $J_{X}$.

(4) $\forall[X] \in \bar{M}_{g}^{0}$, there is a natural action of $J_{X}$ on $\overline{P_{d, X}}$.

Proof. The theorem has almost completely been proved already. For (1), see Theorem 2.1 and Corollary 2.1. For (2) notice that the fibers of $\phi_{d}$ are all $g$ dimensional and the open subset of $\bar{M}_{g}, \bar{M}_{g}^{0}$, is regular, hence the restriction of $\phi_{d}$ to it is flat. ${ }^{6}$ For (3), see Corollary 5.1.

Finally, the proof of (4) is standard: let

$$
\left(\mathscr{L}_{X}, \mathscr{Z}\right) \stackrel{f}{\longrightarrow} V_{X} \stackrel{\pi}{\longrightarrow} \overline{P_{d, X}}
$$

be the restriction of the universal family and of the quotient map to $V_{X}$. Then we can draw a commutative diagram to represent the various morphisms we have:

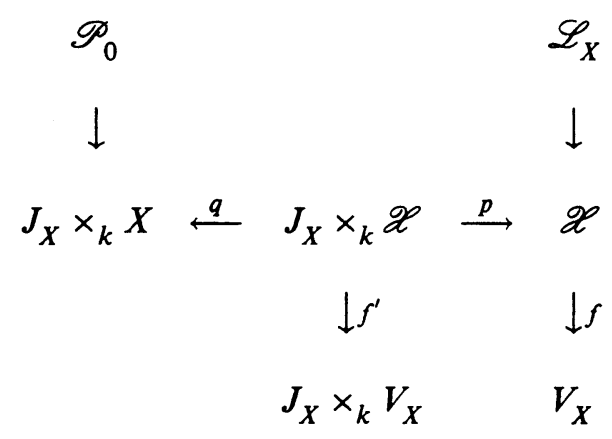

where $f^{\prime}=\mathrm{id}_{J_{X}} \times f, \mathscr{P}_{0}$ is a Poincaré bundle, and $q=p_{J_{X} x_{k} x} \circ s t\left(f^{\prime}\right)$.

Now let

$$
\mathscr{T}=q^{*} \mathscr{P}_{0} \otimes p^{*} \mathscr{L}_{X}
$$

$f_{*}^{\prime} \mathscr{T}$ is locally free, hence we can find an open covering $J_{X} \times_{k} V_{X}=\cup U_{i}$ such that there is a trivialization of $f_{*}^{\prime} \mathscr{T}$ over each $U_{i}$. For each $i$, this gives a

\footnotetext{
${ }^{6}$ We are here applying the following criterion of flatness: let $\phi: X \rightarrow Y$ be a morphism with equi-dimensional fibers; if $X$ is Cohen-Macaulay and $Y$ is regular, then $\phi$ is flat.
} 
morphism

$$
t_{i}: U_{i} \longrightarrow V_{X}
$$

and it is, for all $F \in J_{X}$ and $h \in V_{X}$

$$
t_{i}(F, h)=\operatorname{hilb}\left(X_{h}, F^{*} \otimes L_{h}\right)
$$

where $F^{*}$ is the pull-back of $F$ to $X_{h}$ under the natural map $X_{h} \rightarrow X$. Let

$$
\bar{t}_{i}=\pi \circ t_{i}: U_{i} \longrightarrow \overline{P_{d, X}}
$$

then $\bar{t}_{i}=\bar{t}_{j}$ over $U_{i} \cap U_{j}$, hence we get a morphism

$$
\sigma: J_{X} \times_{k} \overline{P_{d, X}} \rightarrow \overline{P_{d, X}} \text {. }
$$

It is easy to see that $\sigma$ defines an action of $J_{X}$ over $\overline{P_{d, X}}$ and that this action is "natural" in the sense explained in the following remark.

Remark. We know that for every $[X] \in \bar{M}_{g}^{0}$ there exist

$$
\underline{d}^{1}, \ldots, \underline{d}^{\mu^{\prime}} \in B_{X}^{d}, \quad \mu(X) \geq \mu^{\prime},
$$

such that

$$
\left(\overline{P_{d, X}}\right)_{\text {smooth }}=\coprod_{i=1}^{\mu^{\prime}} \mathrm{Pic}_{X}^{d^{i}} .
$$

Now the restriction to every $\operatorname{Pic} \frac{d_{X}^{i}}{x}$ of the action of $J_{X}$ defined by $\sigma$ coincides with the standard action (by tensor product) of $J_{X}$ on Pic ${ }_{X}^{\frac{d^{i}}{}}$.

\subsection{The geometric compactification of the universal Picard variety.}

Proposition 6.2. $\overline{P_{d, g}}$ is a geometric quotient of $H_{d}$ modulo $G$ if and only if $(d-g+1,2 g-2) \stackrel{g}{=}$.

Proof. The above statement can be equivalently formulated by saying that $(d-g+1,2 g-2)=1$ if and only if all points of $H_{d}$ are stable under the action of $G$. The existence of strictly semistable points in $H_{d}$ is equivalent to the existence in $H_{d}$ of points having a positive-dimensional stabilizer. In fact, if a nonstable point $h \in H_{d}$ has finite stabilizer, then its orbit cannot be closed in $H_{d}$; since the closure of an orbit is a union of orbits of smaller dimension, the claim follows.

Therefore, by Lemma 3.2, we can prove the proposition by showing that, with our choice of $d$, and only for that choice, it is impossible for any ladder $X$ that $\operatorname{hilb}(X, L)$ is semistable, for any very ample $L \in \operatorname{Pic}_{X}^{d}$.

Notice that, if $X$ is a ladder, we can decompose $X$ as the union of three subcurves

$$
X=Y_{1} \cup D \cup Y_{2}
$$

so that $Y_{1}$ and $Y_{2}$ are disjoint, $D$ is a union of $k$ destabilizing rational components, and

$$
\left|Y_{1} \cap D\right|=\left|Y_{2} \cap D\right|=k
$$


Let $Y_{1} \cup D=X_{1}$; clearly $X_{1}$ and $Y_{1}$ have the same arithmetic genus, $g_{1}$, and $\left|X_{1} \cap \overline{X \backslash X_{1} \mid}=\right| Y_{1} \cap \overline{X \backslash Y_{1} \mid}=k$. Therefore, the basic inequality imposes exactly the same conditions on $\operatorname{deg}_{X_{1}} L$ and $\operatorname{deg}_{Y_{1}} L$, i.e.,

$$
m_{1} \leq \operatorname{deg}_{X_{1}} L \leq M_{1}
$$

where recall that

$$
m_{1}=\frac{d}{(g-1)}\left(g_{1}-1+k / 2\right)-k / 2 \text { and } M_{1}=m_{1}+k
$$

Since $\operatorname{deg}_{X_{1}}=\operatorname{deg}_{Y_{1}} L+\operatorname{deg}_{D} L=\operatorname{deg}_{Y_{1}} L+k$, we can conclude that

$$
\operatorname{deg}_{X_{1}}=M_{1} \text { and } \operatorname{deg}_{Y_{1}}=m_{1}
$$

hence $m_{1}$ (and $M_{1}$ ) must be integer. Therefore, by Theorem 3.1, the proof of the proposition will follow from the next elementary

Lemma 6.3. $(d-g+1,2 g-2)=1$ if and only if for every complete subcurve $Y$ of $X$, having arithmetic genus $g_{Y}, 0<g_{Y}<g-1$, and intersecting $|\overline{X \backslash Y}|$ in $k$ points, the basic inequality has noninteger extremes, i.e.,

$$
\frac{d}{(g-1)}\left(g_{Y}-1+k / 2\right)-k / 2 \notin \mathbb{Z} \text {. }
$$

Proof of the lemma. We will first show that if $(d-g+1,2 g-2)=1$, then the Basic Inequality cannot assume integer extremes, as long as $0<g_{Y}<g-1$. Suppose that there exist $k, g_{Y}, n \in \mathbb{Z}$ such that

$$
\frac{d}{(g-1)}\left(g_{Y}-1+k / 2\right)-k / 2=n
$$

then

$$
k(d-g+1)-n(2 g-2)=d\left(2-2 g_{Y}\right) .
$$

Now, since $(d-g+1,2 g-2)=1$ we have that $d$ divides $k$, which is impossible.

Viceversa, we want to show that, if the Basic Inequality never assumes integer extremes for all allowed choices of $g_{Y}$ and $k$, then $d-g+1$ and $2 g-2$ are coprime.

First, notice that the assumption implies that $(d, g-1)=1$. In fact for $k=2$ we get

$$
\frac{d}{(g-1)} g_{Y} \notin \mathbb{Z}
$$

for all $g_{Y}$ such that $1 \leq g_{Y} \leq g-2$. Hence $(d, g-1)=1$.

Now, if $(d-g+1,2 g-2)=c \geq 2$, then there exist $a$ and $b$ coprime integers such that

$$
a(d-g+1)+b(2 g-2)=c,
$$

hence

$$
a d+(2 b-a)(g-1)=c
$$

which forces $c=2$. 
Now, there exist two integers $n$ and $r$ such that $d=n(g-1)+r$ with $1 \leq r \leq g-2$ and $(r, g-1)=1$.

Therefore, from

$$
((n-1)(g-1)+r, 2(g-1))=2
$$

we get that $r$ is even, hence $g$ is even and $n$ is odd.

We can now just look at the extremes of the Basic Inequality for $k=3$ and $g_{Y}=a-1$ where $g=2 a$ :

$$
m_{Y}=\frac{n(g-1)+r}{g-1}(a-2+3 / 2)-3 / 2,
$$

hence, $n$ being odd,

$$
m_{Y}=n^{\prime}+\frac{r(2 a-1)}{2(2 a-1)} \in \mathbb{Z},
$$

which is a contradiction.

This concludes the proof of the lemma, as well as the proof of the proposition.

\section{EXAMPLES}

Throughout this section, $X$ will be a DM-stable curve without nontrivial automorphisms.

7.1. Example 1: Irreducible curves. Let $X$ be an irreducible curve with $\delta$ nodes $\left\{p_{1}, \ldots, p_{\delta}\right\}$. If $I \subseteq\{1, \ldots, \delta\}$, we will denote by $X_{I}$ the quasistable curve obtained from $X$ by destabilizing the nodes $p_{i}, \forall i \in I$. There are, of course, $2^{\delta}$ such curves and, for every $I, M_{X_{I}}$ is nonempty and parametrizes projective embeddings of $X_{I}$ having multidegree $(d-|I|, 1,1, \ldots, 1)$. We have

$$
V_{X_{\{1,2, \ldots, \delta\}}} \subset V_{X_{\left\{i_{1}, i_{2}, \ldots, i_{\delta-1}\right\}}} \subset \cdots \subset V_{X_{\left\{i_{1}\right\}}} \subset V_{X}
$$

moreover, $\operatorname{dim} V_{X_{I}}=r(r+2)+g-|I|$ and

$$
V_{X_{I}} / G \cong J_{X^{\nu}}
$$

since a projective realization of $X_{I}$ having semistable multidegree $(d-|I|, 1$, $1, \ldots, 1)$ is uniquely determined by a line bundle of degree $d-|I|$ on $X^{\nu}{ }_{I}$, the partial normalization of $X$ at the nodes corresponding to $I$.

Note also that no point of $V_{X}$ has positive-dimensional stabilizer (cf. 3.3), hence $\overline{P_{d, X}}$ is a geometric quotient of $V_{X}$ modulo $G$.

We can conclude that for irreducible curves the compactified Jacobian $\overline{P_{d, X}}$ will not depend on $d$, it will be irreducible and it will have $\left(\begin{array}{l}\delta \\ k\end{array}\right)$ codimension $k$ irreducible singular loci.

We can globally describe $\overline{P_{d, X}}$ as a moduli space for equivalence classes of line bundles on the curve $X^{\ell}=X_{\{1,2, \ldots, \delta\}}$, whose degree class group has exactly $2^{\delta}$ elements. $^{7}$ Moreover, multidegree classes on $X^{\ell}$ parametrize the

\footnotetext{
${ }^{7}$ See 8.2 for more details about such an equivalence relation.
} 
irreducible components $V_{X_{I}}$ of the strata

$$
U_{|I|=k} V_{X_{1}}
$$

of $V_{X}$.

The singularities of $\overline{P_{d, X}}$ can be described by studying the geometry of $V_{X}$ locally at $M_{X_{I}}$, which can be done by using the results of 2.2 . We conclude that $M_{X_{I}}$ is the transverse intersection of $2|I|$ smooth branches of codimension $|I|-1$, each of which parametrizes deformations of $X_{I}$ in $\mathbb{P}^{r}$ that smooth one of the $2|I|$ unstable nodes of $X_{I}$.

7.2. Example 2: Curves of compact type. A connected, nodal curve $X$ is said to be of compact type if all of its irreducible components are smooth and all of its nodes are disconnecting; this is equivalent to saying that its generalized Jacobian is compact-and hence equal to the product of the Jacobians of the irreducible components of $X$. Let us consider the case in which $X$ has only two irreducible components $C_{1}$ and $C_{2}$, so that $\left|C_{1} \cap C_{2}\right|=1$ and $J_{X}=J_{C_{1}} \times_{k} J_{C_{2}}$. Then the Basic Inequality implies that $B_{X}^{d}$ contains either one or two elements.

If $\left|B_{X}^{d}\right|=1$ we are in the geometric case; the fiber of $H_{d}$ over [X] contains no strictly quasistable curves and no strictly $G$-semistable points. In fact $\psi_{d}^{-1}([X])$ maps onto $J_{X}$ with fibers isomorphic to $P G L(r+1)$.

Let us now consider the case in which the Basic Inequality allows two semistable multidegrees on $X: \underline{d}=\left(m_{1}, M_{2}\right)$ and $\underline{d}^{\prime}=\left(M_{1}, m_{2}\right)=\left(m_{1}+\right.$ $\left.1, M_{2}-1\right)$. Then the multidegree $\left(m_{1}, m_{2}, 1\right)$ is semistable on the ladder of $X$ and there are no other semistable multidegrees (see Figure 4).

What happens is that for every $h=\operatorname{hilb}(X)$ in $\psi_{d}^{-1}([X])$ there exists a point $h^{\ell}=\operatorname{hilb}\left(X^{\ell}\right) \in \psi_{d}^{-1}([X])$ such that $h^{\ell} \in \overline{O_{G}(h)}$. Notice moreover that such an orbit $O_{G}\left(h^{\ell}\right)$ is unique. Finally, $\psi_{d}^{-1}([X])$ has two irreducible components

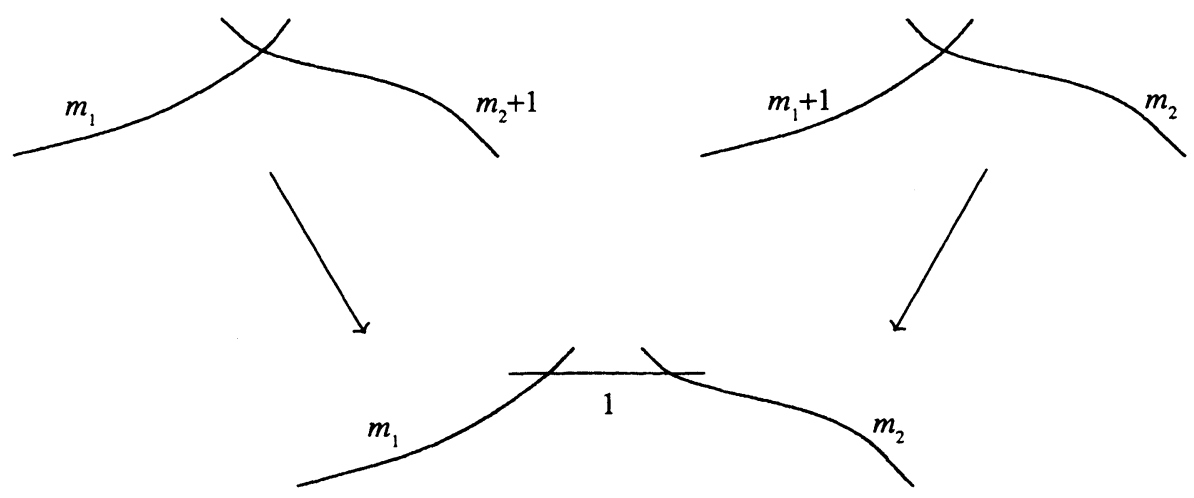

FIGURE 4 
intersecting transversally along the locus of the ladders $V_{X^{\ell}} ; V_{X^{\ell}}$ in turn maps onto $J_{X}$ with fibers isomorphic to quotients of $P G L(r+1)$ modulo $G_{m}$.

In the case of a curve with more than two components, the situation is basically the same; if $B_{X}^{d}$ has more than one element, there exists a unique $G$ semistable ladder $T$ of maximal order, such that all irreducible components of $\psi_{d}^{-1}([X])$ contain $V_{T}$ and such that $V_{T}$ maps surjectively to $J_{X}$ with fibers isomorphic to quotients of $P G L(r+1)$ modulo $\left(G_{m}\right)^{t}$.

7.3. Example 3: Curves with two components. Let $X=C_{1} \cup C_{2}, C_{1}$ and $C_{2}$ smooth and irreducible, $\left|C_{1} \cap C_{2}\right|=k \geq 2$. The generalized Jacobian of $X$, $J_{X}$, is a $\left(k^{*}\right)^{k-1}$-bundle ${ }^{8}$ over the Jacobian of the normalization of $X$. We will now show that our construction yields two different ways of compactifying $J_{X}$.

Recall that $\Delta_{X} \cong \mathbb{Z} / k \mathbb{Z}$. If the extremes of the Basic Inequality for $C_{1}$ (or, equivalently, for $C_{2}$ ) are not integer we will say that we are in the general case for $X$; in such a situation, every degree class will have a unique semistable representative and therefore both $V_{X}$ and $\overline{P_{d, X}}$ will have $k$ irreducible components.

On the other side, the special case will occur when the extremes of the Basic Inequality for $C_{1}$ (and hence $C_{2}$ ) are integer; notice that if

$$
d=(2 h+1)(g-1)
$$

then the special case will occur for every $X$. Now $V_{X}$ has $k+1$ irreducible components, two of which correspond to equivalent multidegrees; in the quotient, these two components will therefore be identified in a positive codimension locus. Finally, $\overline{P_{d, X}}$ will have $k-1$ components.

To examine the situation with more details, we need to simplify the notation. We will reduce the $k$ (respectively $k+1$ ) semistable multidegrees by representing $\left(d_{1}, d_{2}\right)$ by

$$
\left(d_{1}, d_{2}\right) \leadsto\left[d_{1}-\left\lceil m_{1}\right\rceil, d_{2}-\left\lfloor M_{2}\right\rfloor\right]
$$

which corresponds to considering the following set of semistable reduced multidegrees:

$$
\{[0,0],[1,-1], \ldots,[k-1,1-k]\}
$$

(respectively, for the special case

$$
\{[0,0],[1,-1], \ldots,[k-1,1-k],[k,-k]\}) .
$$

We will use the reduced degree of $C_{1}$ for labeling; for example, we will denote by $V_{X}^{j}$ the irreducible component of $V_{X}$ whose generic point corresponds to an embedding of $X$ having reduced multidegree $[j,-j]$, where $0 \leq j \leq k$. Furthermore, if $I \subset\{1,2, \ldots, k\}$, we will denote $V_{I}^{j}=V_{X_{I}}^{j}$, where, as in the previous example, $X_{I}$ denotes the curve obtained from $X$ by destabilizing the nodes $p_{i} \forall i \in I$. To a projective curve isomorphic to $X_{I}$ we will still associate

\footnotetext{
${ }^{8}$ Too many $k$ 's, I apologize for the notation!
} 
a couple $[j,-j-|I|], 0 \leq j \leq k-|I|$, which, by abuse of language, will still be called the "reduced multidegree". As before, such a reduced multidegree is obtained by

$$
\left.\left(d_{1}, d_{2}, 1, \ldots, 1\right)\right) \leadsto\left[d_{1}-\left\lceil m_{1}\right\rceil, d_{2}-\left\lfloor M_{2}\right\rfloor\right] .
$$

In Figures 5 and 6 we give a description of all points of $V_{X}$ if $k=2$ and $k=3$ in both the general and the special case. Under each type of curve, we have all the corresponding semistable reduced multidegrees.

Observe that, in the general case, the boundary points form a closed subset of $V_{X}$ having two disjoint irreducible components. $V_{X}^{0}$ and $V_{X}^{1}$ (the irreducible components of $V_{X}$ ) intersect transversely along these two codimension 1 subvarieties.

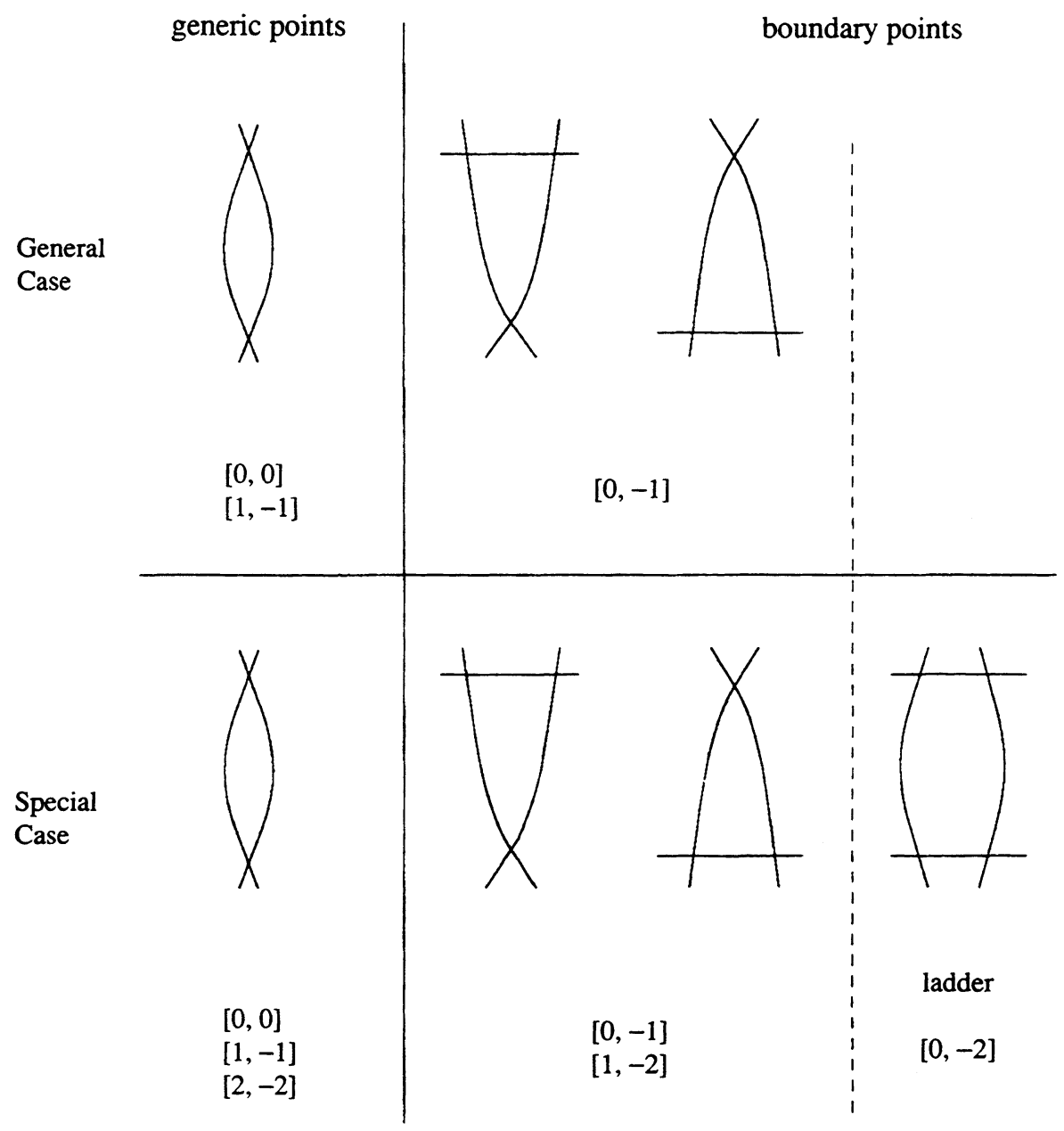

FIGURE 5. $G$-semistable curves for $k=2$ 


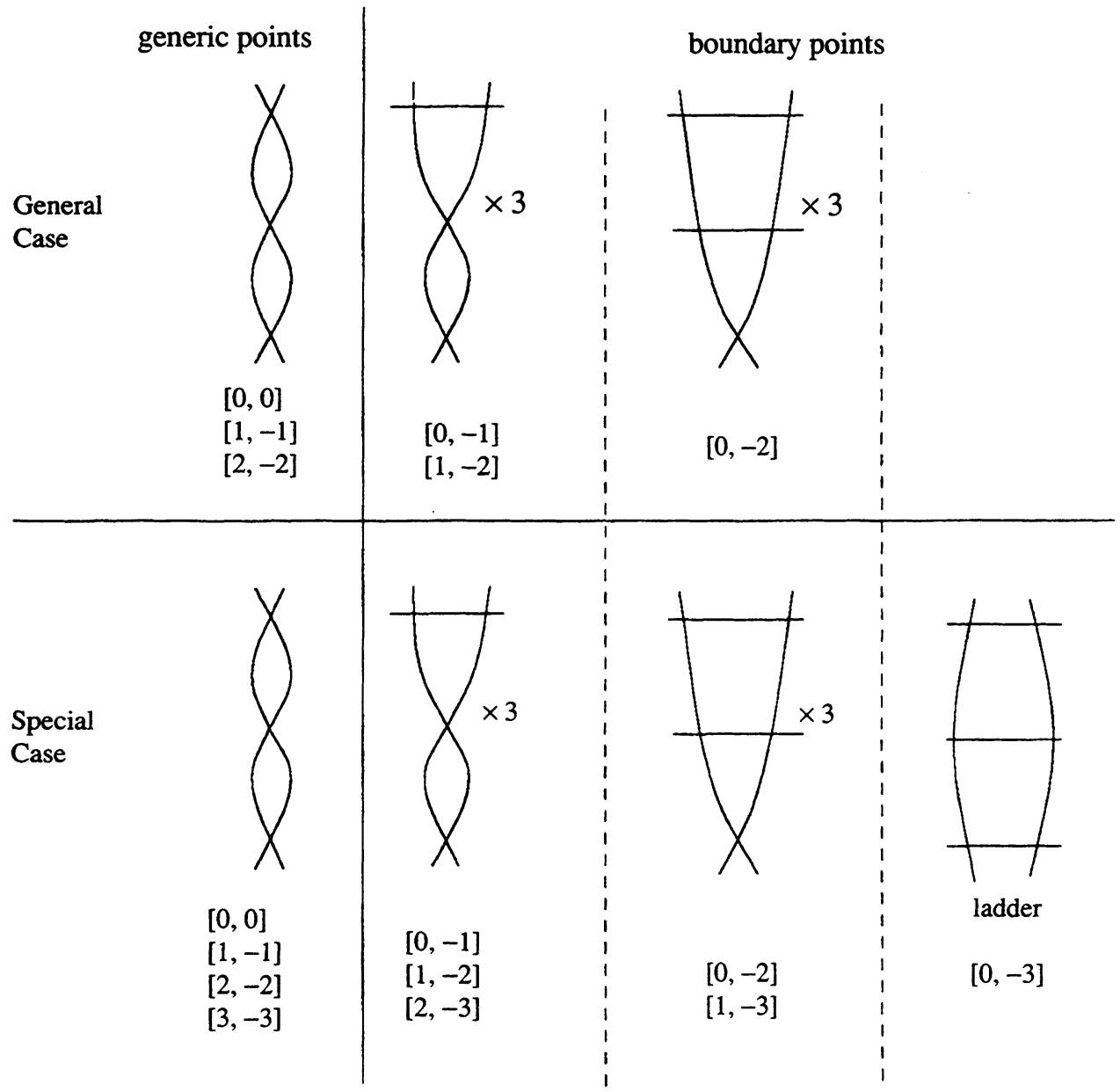

FIGURE 6. $G$-semistable curves for $k=3$

For every $k$, the general case is much simpler to describe, because of the lack of strictly semistable points in $V_{X}$. Proposition 5.1 implies that

$$
V_{I^{\prime}}^{j^{\prime}} \subset V_{I}^{j} \Longleftrightarrow I \subset I^{\prime} \text { and } j-\rho^{\prime}+\rho \leq j^{\prime} \leq j
$$

where $\rho=|I|$ and $\rho^{\prime}=\left|I^{\prime}\right|$. This is really all we need to know to describe how the components of $V_{X}$ intersect.

In the special case, $V_{X}$ contains some points having one-dimensional stabilizer, namely, the Hilbert points of the semistable embeddings of the ladders. We now need the following fact.

Lemma 7.1. Let $X=C_{1} \cup C_{2},\left|C_{1} \cap C_{2}\right|=k$, where $C_{1}$ and $C_{2}$ are smooth and irreducible. Let $h=\operatorname{hilb}(Z, L) \in V_{X}$ be such that $Z$ has $\rho$ destabilizing components and the multidegree of $L$ is $\underline{d}=\left(d_{1}, d_{2}, 1, \ldots, 1\right)$. Then the orbit 
closure of $h$ in $H_{d}$ contains the Hilbert point of a ladder if and only if either $d_{1}=m_{1}$ or $d_{1}=M_{1}-\rho$.

Remark. Of course this lemma has already been proved (cf. 5.2.1 and Lemma 6.1). The ad hoc proof that follows should be viewed as an example illustrating various ideas described in the paper.

Proof. Assume that $d_{1}=m_{1}$; then we will show that $h$ is strictly semistable. (Obviously $d_{1}=M_{1}-\rho$ implies $d_{2}=m_{2}$, hence the proof of the second case is the same.)

It will be enough to exhibit a DM-semistable curve $W$ such that $W$ dominates $Z$ and such that, on $W$, the pull-back of $\underline{d}$ is equivalent to a multidegree $\underline{d}^{\ell} \in B_{Z}$ such that the projective realization of $W$ via any line bundle of multidegree $\underline{d}^{\ell} \in B_{Z}$ is a ladder. Let

$$
Z=C_{1} \cup C_{2} \cup\left(\cup_{j=1}^{\rho} E_{j}\right)
$$

and let

$$
W=C_{1} \cup C_{2} \cup\left(\cup_{j=1}^{\rho} E_{j}\right) \cup\left(\cup_{i=1}^{k} F_{i}\right)
$$

so that $W$ is obtained by adding a rational component $F_{i}$ in place of all the nodes lying on $C_{1}$ (see Figure 7).

On $W$ we have that

$$
E_{j} \cap C_{1}=\varnothing \text { and }\left|E_{j} \cap F_{i}\right|=\delta_{i, j} \forall i, j .
$$

Moreover, it is

$$
\underline{d}^{*}=\left(m_{1}, M_{2}-\rho, d_{E_{j}}^{*}=1, d_{F_{i}}^{*}=0\right) ;
$$

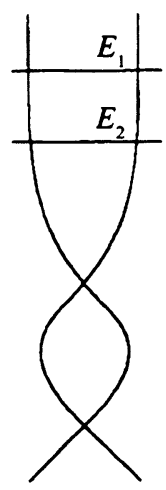

$Z$

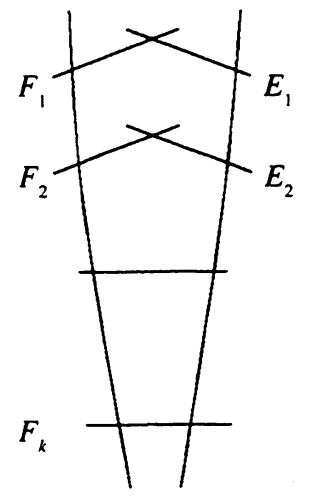

W

FIGURE 7 
now let

$$
\underline{d}^{\ell}=\underline{d}^{*}+\sum_{j=1}^{\rho} \underline{e_{j}}+\underline{c_{2}} .
$$

Clearly

$$
\underline{d}^{\ell}=\left(m_{1}, m_{2}, d_{E_{j}}^{\ell}=0, d_{F_{i}}^{\ell}=1\right),
$$

hence $\underline{d}^{\ell}$ is the degree of a ladder.

Viceversa, we now want to prove that, if $h=\operatorname{hilb}(Z, L)$ is strictly semistable, then either $d_{1}=m_{1}$ or $d_{2}=m_{2}$.

Given the Hilbert point $h^{\ell}$ of any $G$-semistable ladder, there exists $h^{\prime}=$ $\operatorname{hilb}(X)$ such that $h^{\ell}$ is contained in the closure of the orbit of $h^{\prime}$ and such that

$$
\underline{d}^{\prime}=\underline{\operatorname{deg}} X_{h^{\prime}}=\left(m_{1}, M_{2}\right)
$$

(see 5.1.1). Therefore, if

$$
h^{\ell} \in \overline{O_{G}(h)} \cap \overline{O_{G}\left(h^{\prime}\right)}
$$

then, by the semistable replacement property, $\underline{d}^{*} \equiv\left(\underline{d}^{\prime}\right)^{*}$ on some curve dominating $W$. Hence there exists a subcurve $Y$ of $Z$, which is not equal to $C_{1} \cup C_{2}$, and such that $d_{Y}=m_{Y}$. This finishes the proof.

By applying all of this to the case $k=2$, we draw the schematic pictures of $\overline{P_{d, X}}$ in Figure 8.

The case $k=2$ is special in our class of examples, as the singularities of $\overline{P_{d, X}}$ have normal crossing.

Let us now study the case $k=3$; this type of curve is commonly called the "Dollar sign", for reasons that will not be clear from my pictures!

Now there are strata in codimension 2 as well, hence our picture will only represent the compactified fibers of the pull-back morphism $\nu^{*}: J_{X} \longrightarrow J_{X^{\nu}}$ and the way they are glued together.

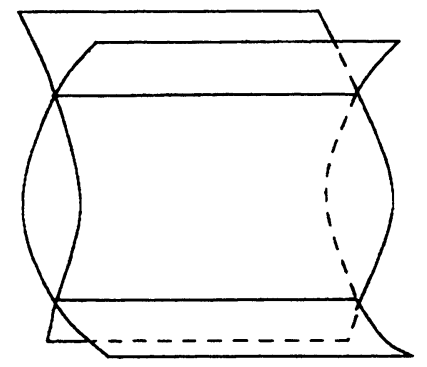

General Case

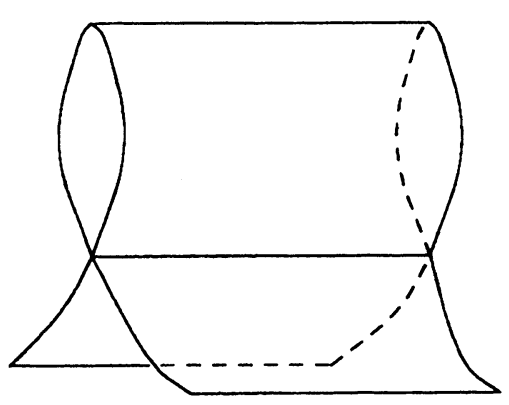

Special Case

Figure 8 
We now denote by $P_{i}$ the irreducible component of $\overline{P_{d, X}}$ which contains an open subset parametrizing line bundles of reduced multidegree $[i,-i]$. We already saw that, in the general (resp. special) case, $\overline{P_{d, X}}$ has three (resp. two) components, each of which compactifies a $k^{*}$-bundle over $J_{X^{\nu}}$.

Figure 9 represents the three projective surfaces which compactify the fibers of the pull-back morphism, in the canonical model of the compactified Jacobian. In $P_{0}$ and $P_{2}$ the fibers are compactified by $\mathbb{P}^{2}$, in $P_{1}$ by $\mathbb{P}^{2}$ blown up at three points, where equal symbols denote identified loci. These loci parametrize projective curves as described in Figure 10 on the next page (we are omitting the degree of the destabilizing component, which is always 1 ).

Figure 11 on the next page corresponds to the degenerate case, in which $\overline{P_{d, X}}$ only has two irreducible components.

The symbols A, B, C correspond to the same objects as in the general case. The new symbol (the grey circle) stands for the locus of the ladders, in which the two components $V_{0}$ and $V_{3}$ get contracted. Notice that the codimension 2 locus gives a worse singularity than before.

We remark that a picture analogous to the one that we just described appears in $[\mathrm{OS}]$.
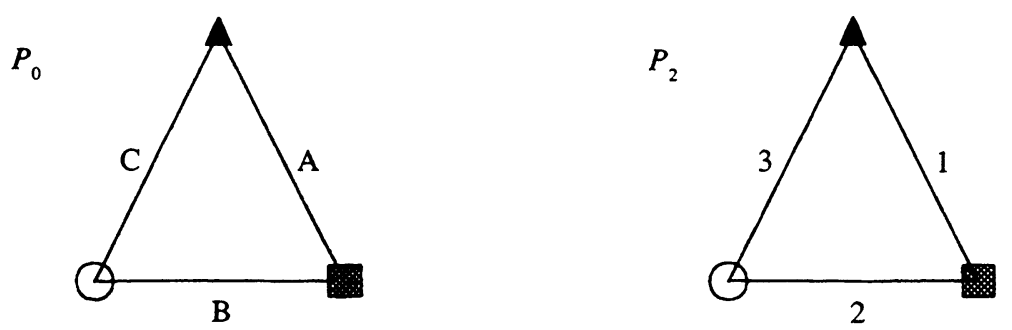

General

Case

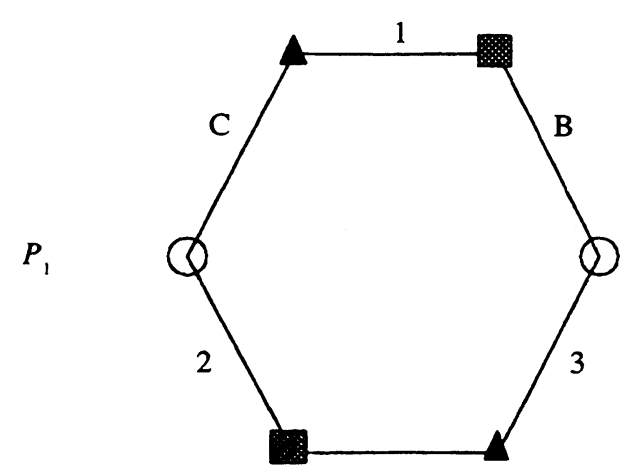

A

FIGURE 9 
$1:$<smiles>CC(C)(C)C1CC1C(C)(C)C</smiles>

A:

$\underbrace{[1,-2]}$
2:

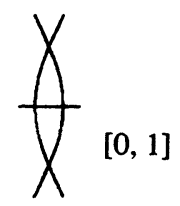

B:

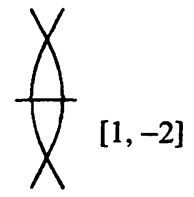

$\Delta$ :

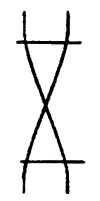

3:<smiles></smiles>

C:

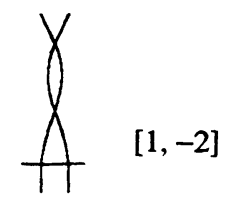

Figure 10
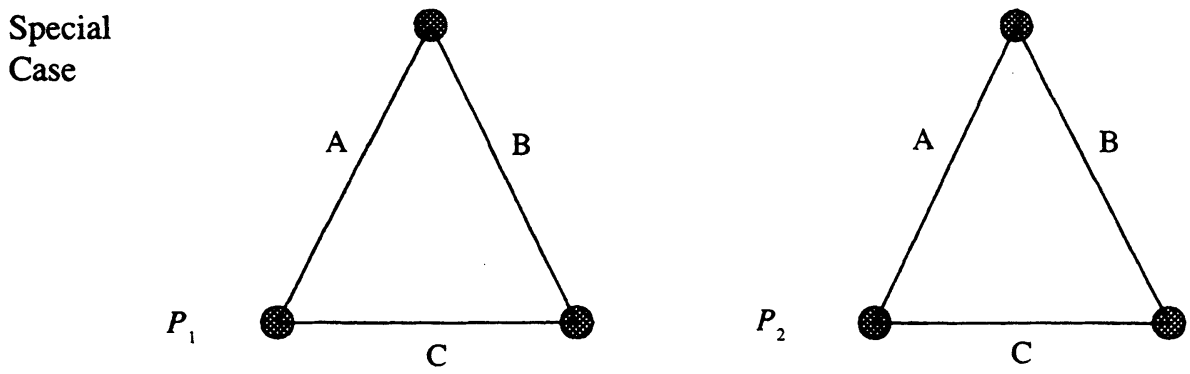

Figure 11

\section{THE MODULI PROBLEM}

In this section, we describe the schemes $\overline{P_{d, g}}$ and $\overline{P_{d, X}}$ in the language of functors and we study their dependence on the degree $d$.

8.1. The functor of line bundles with balanced multidegree on a family. We start by defining a functor $\overline{\mathscr{P}_{d, g}}$, following what the Geometric Invariant Theory suggests, and then we say when and how $\overline{P_{d, g}}$ represents it. The purpose of this work was to compactify the degree $d$ Picard functor, $\mathscr{P}_{d, g}$, for families of regular curves; it is clear that the functor defined below contains $\mathscr{P}_{d, g}$.

Definition. We define the contravariant functor $\overline{\mathscr{P}_{d, g}}$ from schemes to sets, to be such that for every scheme $S, \overline{\mathscr{P}_{d, g}}(S)$ is the set of equivalence classes of 
polarized families of quasistable curves of genus $g$,

$$
f:(\mathscr{Y}, \mathscr{L}) \longrightarrow S,
$$

such that $\mathscr{L}$ is a relatively very ample line bundle of degree $d$, whose multidegree satisfies the Basic Inequality on each fiber; here we say that two families over $S,(\mathscr{Y}, \mathscr{L})$ and $\left(\mathscr{Y}^{\prime}, \mathscr{L}^{\prime}\right)$, are equivalent if there exists an $S$ isomorphism

$$
\alpha: \mathscr{Y} \longrightarrow \mathscr{Y}^{\prime}
$$

and a line bundle $M$ on $S$, such that

$$
\alpha^{*} \mathscr{L}^{\prime} \cong \mathscr{L} \otimes f^{*} M
$$

If $h: S \rightarrow S^{\prime}$ is a morphism of schemes, then

$$
\overline{\mathscr{P}_{d, g}}(h): \overline{\mathscr{P}_{d, g}}\left(S^{\prime}\right) \longrightarrow \overline{\mathscr{P}_{d, g}}(S)
$$

is given by using $h$ to pull back to $S^{\prime}$ polarized families on $S$.

Then the result is

Proposition 8.1. (1) There exists a morphism of functors

$$
\Xi: \overline{\mathscr{P}_{d, g}} \longrightarrow \mathscr{H} \text { om }\left(, \overline{P_{d, g}}\right)
$$

inducing an injection of the subfunctor $\mathscr{P}_{d, g}$ into $\mathscr{H}$ om $\left(, P_{d, g}\right)$ such that $P_{d, g}$ coarsely represents $\mathscr{P}_{d, g}$.

(2) $\overline{P_{d, g}}$ coarsely represents $\overline{\mathscr{P}_{d, g}}$ if and only if $(d-g+1,2 g-2)=1$.

(3) If $(d-g+1,2 g-2)=1$, then $P_{d, g}$ is a fine moduli scheme for $\mathscr{P}_{d, g}$. Proof. The proof is standard Geometric Invariant Theory and it is very similar to that of Theorem 2.1.

(1) The morphism $\Xi$ is constructed by using the functorial property of $\mathrm{Hilb}_{r}^{p(x)}$ : let $\mathscr{F}$ be an element of $\overline{\mathscr{P}_{d, g}}(S)$ and let the polarized family

$$
(\mathscr{Y}, \mathscr{L}) \stackrel{f}{\longrightarrow} S
$$

be a representative for $\mathscr{F}$. Then there is a covering $S=\cup U_{i}$ such that the restriction of the polarized family to each $U_{i}$ gives a morphism $t_{i}$ to $\mathrm{Hilb}_{r}^{p(x)}$. Since the relative multidegree of $\mathscr{L}$ satisfies the Basic Inequality, the image of $t_{i}$ is in $H_{d} \cdot t_{i}\left(U_{i} \cap U_{j}\right)$ and $t_{j}\left(U_{i} \cap U_{j}\right)$ are clearly congruent (in an algebraic way) modulo $G$, that is, the compositions $\bar{t}_{i}=\pi_{d} t_{i}{ }^{9}$ can be glued together to a morphism

$$
\Xi(S)(\mathscr{F}): S \longrightarrow \overline{P_{d, g}}
$$

which clearly does not depend on the choice of the $t_{i}$.

The restriction of $\Xi$ to families of smooth curves gives an injection of $\mathscr{P}_{d, g}(S)$ into $\mathscr{H} \operatorname{om}\left(S, \overline{P_{d, g}}\right)$ simply because $\forall[X] \in \bar{M}_{g}$ and $\forall L \in \operatorname{Pic}_{X}^{d}$, $\operatorname{hilb}(X, L)$ is $G$-stable.

\footnotetext{
${ }^{9}$ Recall that $\pi_{d}: H_{d} \rightarrow \overline{P_{d, g}}$ is the quotient morphism.
} 
Notice that while $P_{d, g}$ is equal to $\phi_{d}^{-1}\left(M_{g}^{0}\right)$ the argument above applies to smooth curves with automorphisms as well, because of the way we defined the equivalence relation on polarized families. The remaining part of (1) has been proved in Theorem 2.1 already.

(2) Assume $(d-g+1,2 g-2)=1$. Saying that $\overline{P_{d, g}}$ coarsely represents $\overline{\mathscr{P}_{d, g}}$ is precisely saying that the following two properties are satisfied:

(i) For every algebraically closed field $K$, the map

$$
\Xi(\operatorname{Spec} K): \overline{\mathscr{P}_{d, g}}(\operatorname{Spec} K) \longrightarrow \mathscr{H} o m\left(\operatorname{Spec} K, \overline{P_{d, g}}\right)
$$

is an isomorphism.

(ii) Given any scheme $P$ such that there is a morphism of functors

$$
\Upsilon: \overline{\mathscr{P}_{d, g}} \longrightarrow \mathscr{H} \text { om }(, P)
$$

there exists a unique morphism

$$
\psi: \overline{P_{d, g}} \longrightarrow P
$$

such that

$$
\Upsilon=\Psi \circ \Xi
$$

( $\Psi$ being the morphism of functors $\mathscr{H} o m\left(, \overline{P_{d, g}}\right) \rightarrow \mathscr{H} o m(, P)$ given by composition by $\psi$ ).

The proof of (i) follows directly from Proposition 6.2. Let us prove (ii). To construct $\psi$, we look at the image under

$$
\Upsilon\left(H_{d}\right): \overline{\mathscr{P}_{d, g}}\left(H_{d}\right) \longrightarrow \mathscr{H} \text { om }\left(H_{d}, P\right)
$$

of the class of the universal polarized family $\mathscr{U}$,

$$
\left(\mathscr{X}_{d}, \mathscr{L}_{d}\right) \longrightarrow H_{d}
$$

This will give a morphism $\psi^{\prime}: H_{d} \rightarrow P$, which will be $G$-invariant, because of the way we defined the equivalence relation among families. But now, by the universal property of the GIT-quotient, $\psi^{\prime}$ can be canonically factored through a unique $\psi: \overline{P_{d, g}} \rightarrow P$ such that $\psi^{\prime}=\psi \circ \pi_{d}$.

Remark. The "maximality" property (ii) holds for every $d$, in fact there is no need for the assumption $(d-g+1,2 g-2)=1$.

Viceversa, assume that $\overline{P_{d, g}}$ is a coarse moduli scheme for $\overline{\mathscr{P}_{d, g}}$; then its points have to be in one-to-one correspondence with equivalence classes of very ample line bundles of degree $d$ on quasistable curves, satisfying the Basic Inequality. This can of course only happen if $\overline{P_{d, g}}$ is a geometric quotient of $H_{d}$ modulo $G$. By Proposition 6.2, we conclude that $(d-g+1,2 g-2)=1$.

Finally, the proof of (3) is a simple application of a general construction due to Maruyama (see [Ma]).

Let $H_{d}^{0}:=\psi^{-1}\left(M_{g}^{0}\right)$ as in the proof of Theorem 2.1 , and let $\left(\mathscr{L}_{0}, \mathscr{Z}_{0}\right)$ be the restriction of the universal polarized family to it. Then we have the following 
diagram:

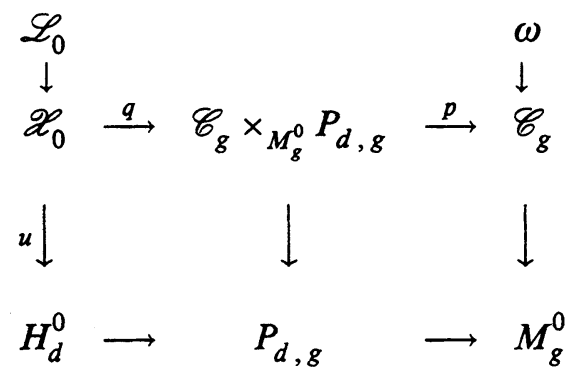

where $\omega$ is the relative dualizing sheaf (represented here as a line bundle) of the universal family over $M_{g}^{0}$. We want to find a line bundle $F$ on $H_{0}$ such that $\mathscr{L}_{0} \otimes u^{*} F$ can be descended to a line bundle $\mathscr{P}$ on $\mathscr{C}_{g} \times_{M_{g}^{0}} P_{d, g}$. It is clear that, if such an $F$ exists, $\mathscr{P}$ will be a Poincare line bundle, and the proof of (3) will be finished.

By our assumption, there exist integers $a$ and $b$ such that

$$
a(d-g+1)+b(2 g-2)=-1,
$$

hence the number $n=b-(a-1) / 2$ is an integer. Let us now define

$$
F:=\operatorname{det}\left(u_{*}\left(\mathscr{L}_{0}^{\otimes a} \otimes q^{*} p^{*} \omega^{\otimes n}\right)\right) ;
$$

then, if an element of $G$ acts on $\mathscr{L}_{0}$ via multiplication by the constant $\alpha$, the same element will act on $F$ via multiplication by $\alpha^{-1}$; hence $\mathscr{L}_{0} \otimes u^{*} F$ admits a linearization which is compatible with the action of $G$ on $H_{d}^{0}$. In other words, $\mathscr{L}_{0} \otimes u^{*} F$ can be descended to a Poincaré line bundle on $\mathscr{C}_{g} \times_{M_{g}^{0}} P_{d, g}$.

Remark. The converse of (3) is proved in the paper [MR], where there is also a proof of (3) over the field of complex numbers.

We now want to study the relation between compactifications obtained for different degrees.

Lemma 8.1. There exists an isomorphism

$$
\tau: \overline{P_{d, g}} \longrightarrow \overline{P_{d^{\prime}, g}}
$$

if and only if there exists $n$ such that $d \pm d^{\prime}=n(2 g-2)$.

Proof. If $d \pm d^{\prime}=n(2 g-2)$, then notice that the functors $\overline{\mathscr{P}_{d, g}}$ and $\overline{\mathscr{P}_{d^{\prime}, g}}$ are isomorphic. In fact, assume that $d+d^{\prime}=2 g-2$ (the other case is similar); then one can define a morphism

$$
\overline{\mathscr{P}_{d, g}}(S) \longrightarrow \overline{\mathscr{P}_{d, g}}(S)
$$

by sending the polarized family

$$
((\mathscr{L}, \mathscr{Y}) \longrightarrow S) \in \overline{\mathscr{P}_{d, g}}(S)
$$


to

$$
\left(\left(\mathscr{L}^{-1} \otimes \omega_{\mathscr{Y} \mid S}^{\otimes n}, \mathscr{Y}\right) \longrightarrow S\right) \in \overline{\mathscr{P}_{d^{\prime}, g}}(S) .
$$

It is easy to see that this is in fact an isomorphism; now, by the remark in the proof of the previous proposition, we know that $\overline{P_{d, g}}$ and $\overline{P_{d^{\prime}, g}}$ satisfy the maximality property for the respective functors, hence they are isomorphic.

The converse is immediate, as it is true for the universal Picard variety. We recall here that the proof follows from the two following well-known facts:

(a) For a general smooth curve $X$, the automorphism group of $J_{X}$ is generated by the involution and the translations.

(b) If $e$ is an integer such that there exists a rational section of $P_{e, g} \rightarrow M_{g}^{0}$, then $e$ is a multiple of $2 g-2$.

8.2. The restriction to a fixed curve. Let us now fix a DM-stable curve $X$. In a completely analogous way as we did before, we can construct a functor $\overline{\mathscr{P}_{d, X}}$ of line bundles with degree restrictions on quasistable curves having $X$ as stable model. The next result is proved like the analogous result for $\overline{P_{d, g}}$ that we have already described.

Proposition 8.2. Let $X$ be a DM-stable curve.

(1) There exists a morphism of functors

$$
\Xi_{X}: \overline{\mathscr{P}_{d, X}} \longrightarrow \mathscr{H} \text { om }\left(, \overline{P_{d, X}}\right) .
$$

(2) $\overline{P_{d, X}}$ coarsely represents $\overline{\mathscr{P}_{d, X}}$ if and only if $d$ is $X$-general.

Another, perhaps more suggestive, description of $\overline{P_{d, X}}$ can be given in terms of line bundles over the ladder $X^{\ell}$, in a way that does not involve the Basic Inequality at all. Nevertheless such a description is intrinsically rigid, that is, it cannot possibly be extended to a family, because it depends on the datum of a deformation of the curve.

For simplicity, let us assume that the automorphism group of the curve is trivial. Let us fix the following datum $\xi$ :

$\xi$ is the datum of a one-parameter deformation of $X^{\ell}$

$$
X^{\ell} \stackrel{i}{\leftrightarrow} \mathscr{Z} \longrightarrow\left(B, b_{0}\right)
$$

where $\mathscr{Z}, B, Z_{b}$ for all $b \neq b_{0}$ are regular, and $i$ is the isomorphism between $X^{\ell}$ and the central fiber $Z$.

Now let $C_{1}, \ldots, C_{\gamma}$ be the irreducible components of $X^{\ell}$; for every $\underline{n}=$ $\left(n_{1}, \ldots, n_{\gamma}\right) \in \mathbb{Z}^{\gamma}$ we have a unique line bundle on $X^{\ell}$

$$
T_{\xi, \underline{n}}=\mathscr{O}_{\mathcal{Z}}\left(\sum_{1}^{\gamma} n_{i} C_{i}\right) \otimes \mathscr{O}_{Z} .
$$

We define the following functor. 
Definition. Let $\mathscr{F}_{\xi}^{d}$ be the contravariant functor from schemes to sets such that, if $S$ is a scheme, $\mathscr{F}_{\xi}^{d}(S)$ is the set of $\xi$-equivalence classes of line bundles $\mathscr{L}$ of relative degree $d$ on the family $X^{\ell} \times{ }_{k} S \longrightarrow S$. If $f: S^{\prime} \longrightarrow S$ is a morphism, then $\mathscr{F}_{\xi}^{d}(f)$ is given by pull-back. Here we say that $\mathscr{L}$ is $\xi$-equivalent to $\mathscr{L}^{\prime}$ if and only if there exist an $S$-automorphism $\alpha$ of $X^{\ell} \times_{k} S$, a line bundle $M$ on $S$, and a $T_{\xi, \underline{n}}$ such that

$$
\mathscr{L}^{\prime} \cong \alpha^{*} \mathscr{L} \otimes p_{S}^{*} M \otimes p_{X^{\prime}}^{*} T_{\xi, \underline{n}} .
$$

Now the relation with $\overline{P_{d, X}}$ is precisely expressed by the next lemma.

Lemma 8.2. (1) There exists a morphism of functors

$$
\Gamma: \mathscr{F}_{\xi}^{d} \longrightarrow \mathscr{H} \text { om }\left(, \overline{P_{d, X}}\right) .
$$

(2) If $d$ is $X$-general, for every algebraically closed field $K$ the map

$$
\Gamma(\operatorname{Spec} K): \mathscr{F}_{\xi}^{d}(\operatorname{Spec} K) \longrightarrow \mathscr{H} \text { om }\left(\operatorname{Spec} K, \overline{P_{d, X}}\right)
$$

is bijective.

The proof is another variation of the proof of Theorem 2.1.

Notice that, even if $d$ is $X$-general, $\overline{P_{d, X}}$ is not even a coarse moduli scheme for $\mathscr{F}_{\xi}^{d}$, for it does not satisfy the maximality property. This is not surprising, as the definition of our $\xi$-equivalence relation on line bundles on $X^{\ell}$ depends on the deformation data $\xi$. Moreover, observe that for any $d$ and $d^{\prime}, \mathscr{F}_{\xi}^{d}$ is clearly isomorphic to $\mathscr{F}_{\xi}^{d^{\prime}}$, whereas we are now going to show that, in general, there does not exist a morphism between $\overline{P_{d, X}}$ and $\overline{P_{d^{\prime}, X}}$.

Suppose for simplicity that $d$ and $d^{\prime}$ are both $X$-general. Then by Proposition 5.1 and Theorem 3.1, if $\overline{P_{d, X}} \cong \overline{P_{d^{\prime}, X}}$ then there exists $\underline{l}=\left(l_{1}, \ldots, l_{\gamma}\right)$ with $\sum l_{i}=d^{\prime}-d$ such that $\forall \underline{d} \in B_{X}^{d}, \underline{l}+\underline{d} \in B_{X}^{d^{\prime}}$. Viceversa, if there exists $\underline{l}=\left(l_{1}, \ldots, l_{\gamma}\right)$ such that $\underline{d}+B_{X}^{\underline{d}}=B_{X}^{d^{\prime}}$, then we obtain the isomorphism between $\overline{P_{d, X}}$ and $\overline{P_{d^{\prime}, X}}$ by tensoring by a line bundle of multidegree $\underline{l}$. An example when this fails to happen is a curve of genus 4 having three smooth components of genus 1 , meeting pairwise in one point. If $d=3 n+1$ and $d^{\prime}=3 n+2$ we have

$$
\overline{P_{d, X}} \not \overline{P_{d^{\prime}, X}} .
$$

To conclude, we can summarize the situation as follows:

Proposition 8.3. Let $d$ be $X$-general and let $d^{\prime} \geq d$; there exists a $J_{X}$-morphism

$$
f: \overline{P_{d, X}} \longrightarrow \overline{P_{d^{\prime}, X}}
$$

if and only if there exists a decomposition in disjoint sets

$$
I \cup J=\{1, \ldots, \gamma\}
$$


such that

$$
\underline{d^{\prime}}-\underline{d}=\sum_{i \in I}\left(\left\lceil m_{i}^{\prime}\right\rceil-\left\lceil m_{i}\right\rceil\right)+\sum_{j \in J}\left(\left\lfloor M_{j}^{\prime}\right\rfloor-\left\lfloor M_{j}\right\rfloor\right) .
$$

Proof. If there exists such a $J_{X}$-morphism $f$, then there has to exist an $\underline{l}=$ $\left(l_{1}, \ldots, l_{\gamma}\right)$ such that

$$
\sum_{1}^{\gamma} l_{i}=\underline{d^{\prime}}-\underline{d}
$$

and such that

$$
B_{X}^{d}+\underline{l} \subset B_{X}^{d^{\prime}}
$$

In fact, since $d$ is $X$-general,

$$
\left(\overline{P_{d, X}}\right)_{\text {smooth }}=\coprod_{\underline{d} \in B_{X}^{d}} \operatorname{Pic}_{X}^{d} .
$$

Now, $\forall \underline{d} \in B_{X}^{d}, f$ maps $\operatorname{Pic}_{X}^{\frac{d}{X}}$ isomorphically to

$$
\operatorname{Pic}_{X}^{d^{\prime}} \subset \overline{P_{d^{\prime}, X}}
$$

therefore, there exists $\underline{l}$ such that $\sum_{1}^{\gamma} l_{i}=\underline{d^{\prime}}-\underline{d}$. As we said, $\underline{l}$ does not depend on the choice of $\underline{d}$.

Now, the fact that

$$
\underline{d^{\prime}}-\underline{d}=\sum_{i \in I}\left(\left\lceil m_{i}^{\prime}\right\rceil-\left\lceil m_{i}\right\rceil\right)+\sum_{j \in J}\left(\left\lfloor M_{j}^{\prime}\right\rfloor-\left\lfloor M_{j}\right\rfloor\right)
$$

follows from the observation that, for every $i, \quad 1 \leq i \leq \gamma$, there exists a multidegree $\left(d_{1}, \ldots, d_{\gamma}\right) \in B_{X}^{d}$ such that $d_{i}=\left\lceil m_{i}\right\rceil$. Now, if

$$
l_{i} \neq\left\lceil m_{i}^{\prime}\right\rceil-\left\lceil m_{i}\right\rceil
$$

(which can only happen if $m_{i}^{\prime} \in \mathbb{Z}$ ) then, a fortiori,

$$
l_{i}=\left\lfloor M_{i}^{\prime}\right\rfloor-\left\lfloor M_{i}\right\rfloor \text {. }
$$

Conversely, given an $\underline{l}$ as in our statement, let $L \in \operatorname{Pic}_{X}^{l}$ and then consider the polarization

$$
q^{*} p_{X}^{*} L \otimes \mathscr{L}_{X}=\mathscr{T}
$$

over the restriction of the universal family $\mathscr{C}_{X}$ to $V_{X}$ :

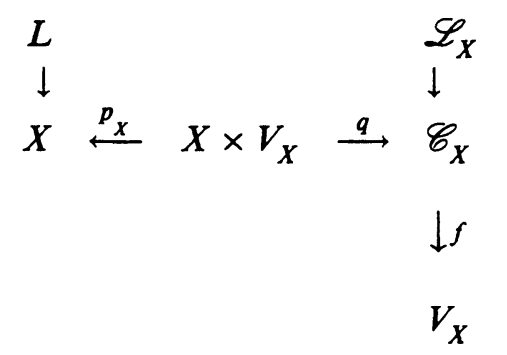


where $\mathscr{L}_{X}$ is the tautological polarization. The relative multidegree of $\mathscr{T}$ clearly satisfies the Basic Inequality, hence we obtain a $G$-invariant morphism $V_{X} \rightarrow \mathrm{Pic}_{X}^{d^{\prime}}$ which, by the universal property of the GIT-quotient, gives the morphism $f$ from $\overline{P_{d, X}}$ to $\overline{P_{d^{\prime}, X}}$, which is what we wanted.

\section{ACKNOWLEDGMENTS}

The subject of this paper is my Ph.D. thesis; I am very grateful to my advisor Joe Harris, who introduced me to the problem of compactifying the universal Picard variety and gave me endless advice with enormous enthusiasm and patience. I could not have done this work without his support. It is also a great pleasure to thank Aaron Bertram and Kieran O'Grady for many helpful conversations and much encouragement; and Ron Donagi, David Mumford, and Angelo Vistoli from whose comments I very much benefitted. My sincere thanks to Dan Abramovic and Ian Morrison, who read a former version of this paper and offered many valuable suggestions, on everything from English mistakes to issues of mathematical accuracy. Finally, I thank the referee of the paper for his very detailed report in which he remarked on some incorrect statements, suggesting ways to adjust them. In particular, he provided the argument for Lemma 5.1, to fix my original proof which had a gap; and the material in Section 5.3 owes a great deal to his contribution.

\section{REFERENCES}

[AK1] A. Altman and S. Kleiman, Compactifying the Picard scheme, Adv. in Math. 35 (1980), 50-112.

[AK2] Compactifying the Picard scheme II, Amer. J. Math. 101 (1979), $10-41$.

[ACGH] E. Arbarello, M. Cornalba, P. A. Griffiths, and J. Harris, Geometry of algebraic curves, Springer-Verlag, Berlin and New York, 1985.

[DM] P. Deligne and D. Mumford, The irreducibility of the space of curves of given genus, Inst. Hautes Études Sci. Publ. Math. 36 (1969), 75-109.

[Ds] C. D'Souza, Compactification of generalized Jacobian, Proc. Indian Acad. Sci. Sect. A Math. Sci. 88 (1979), 419-457.

[DN] J. M. Drezet and M. S. Narasimhan, Groupe de Picard des variétés de modules de fibrés semi-stables sur les courbes algébriques, Invent. Math. 97 (1989), 55-94.

[EC] F. Enriques and O. Chisini, Teoria geometrica delle equazioni delle funzioni algebriche, Vol. 3, Bologna.

[G] D. Gieseker, Lectures on moduli of curves, Tata Inst. Fund. Res. Lectures on Math. and Phys., vol. 69, Springer-Verlag, Berlin and New York, 1982.

[G1] Geometric Invariant Theory and application to moduli problems, Invariant Theory (Montecatini 1982), Lecture Notes in Math., vol. 996, Springer-Verlag, Berlin and New York, 1982.

[G2] _ A degeneration of the moduli space of stable bundles, J. Differential Geom. 19 (1984), 173-206.

[GH] P. A. Griffiths and J. Harris, Principles of algebraic geometry, Wiley, New York, 1978.

[Hi] J. Harris, Algebraic geometry: A first course, Springer-Verlag, Berlin and New York, 1992.

[HM] J. Harris and I. Morrison, Notes on moduli of curves and parameter spaces, 1992, unpublished.

[Ho] R. Hartshorne, Algebraic geometry, Springer-Verlag, Berlin and New York, 1977. 
[HR] M. Hochster and J. Roberts, Rings of invariants of reductive groups acting on regular rings are Cohen-Macaulay, Adv. in Math. 13 (1974).

[Ig] J. Igusa, Fiber systems of Jacobian varieties, Proc. Internat. Symposium on Algebraic Geometry (Kyoto 1977), Kinokuniya Bookstore, Tokyo, 1978, pp. 503-524.

[Is] M. Ishida, Compactifications of a family of generalized Jacobian varieties, Proc. Internat. Symposium on Algebraic Geometry (Kyoto 1977), Kinokuniya Bookstore, Tokyo, 1978, pp. 503-524.

[KI] S. Kleiman, The structure of the compactified Jacobian: A review and announcement, preprint.

[Ko] J. Kollar, Projectivity of complete moduli, J. Differential Geom. 32 (1990).

[LS] S. Lichtenbaum and M. Schlessinger, The cotangent complex of a morphism, Trans. Amer. Math. Soc. 128 (1967), 41-70.

[Ma] M. Maruyama, Moduli of stable sheaves II, J. Math. Kyoto Univ. 18 (1978).

[MR] N. Mestrano and S. Ramanan, Poincaré bundles for families of curves, J. Reine Angew. Math. 362 (1985), 169-178.

[M1] D. Mumford, Lectures on curves on an algebraic surface, Ann. of Math. Studies, no. 59, Princeton Univ. Press, Princeton, NJ, 1966.

[M2] _ Curves and their Jacobians, Univ. of Michigan Press, Ann Arbor, MI, 1975.

[MM] Stability of projective varieties, Enseign. Math. (2) 23 (1977), 39-1 10.

[GIT] D. Mumford and J. Fogarty, Geometric Invariant Theory, Springer-Verlag, Berlin and New York, 1982.

[N] P. E. Newstead, Introduction to moduli problems and orbit spaces, Tata Inst. Fund. Res. Lectures on Math. and Phys., vol. 51, Narosa, New Delhi, 1978.

[OS] T. Oda and C. Seshadri, Compactifications of the generalized Jacobian variety, Trans. Amer. Math. Soc. 253 (1979), 1-90.

[Sn] E. Sernesi, On the existence of certain families of curves, Invent. Math. 75 (1984), $25-57$.

[Se] J. P. Serre, Groupes algébriques et corps de classes, Hermann, Paris, 1959.

Department of Mathematics, Harvard University, Cambridge, Massachusetts 02138

E-mail address: caporasoezariski.harvard.edu 